\title{
Kinetic Monte Carlo (KMC) Algorithm for Nanocrystals
}

by

Sione Paea

\author{
A thesis \\ submitted to the Victoria University of Wellington \\ in fulfilment of the \\ requirements for the degree of \\ Doctor of Philosophy \\ in Mathematics.
}

Victoria University of Wellington

2011 


\begin{abstract}
This thesis uses the kinetic Monte Carlo (KMC) algorithm to examine the growth morphology and structure of nanocrystals. Crystal growth in a supersaturated gas of atoms and in an undercooled binary melt is investigated. First, in the gas phase, the interplay of the deposition and surface diffusion rates is studied. Then, the KMC algorithm is refined by including solidification events and finally, by adding diffusion in the surrounding liquid.

A new algorithm is developed for modelling solidification from an undercooled melt. This algorithm combines the KMC method, which models the change in shape of the crystal during growth, with a macroscopic continuum method that tracks the diffusion of material through solution towards the crystal. For small length and time scales, this approach provides simple, effective front tracking with fully resolved atomistic detail of the crystal-melt interface. Anisotropy is included in the model as a surface diffusion process and the growth rate of the crystal is found to increase monotonically with increase in the surface anisotropy value. The method allows for the study of multiple crystal nuclei and Ostwald ripening. This method will aid researchers to explain why certain crystal shapes form under particular conditions during growth, and may enable nanotechnologists to design techniques for growing nanocrystals with specific shapes for a variety of applications, from catalysis to the medicine field and electronics industry. This will lead to a better understanding of the atomistic process of crystal growth at the nanoscale.
\end{abstract}


This thesis is dedicated to my parents Lata and Tesimoni Paea

of

Mo'unga'one Ha'apai, Tonga. 


\section{Acknowledgments}

I wish to sincerely thank Professor Shaun Hendy for his supervision of this study, particularly for his valuable feedback on theoretical and practical aspects of the research. This acknowledgment cannot ignore his extra financial and learning support, guidance, inspiration and encouragement during the course of this study. Ultimately, his critical criticism and editorial added in depth to correct any incompleteness as a whole and his foresight helped structure this thesis. Thanks also to the co-supervisor of this study, Associate Professor Mark McGuinness, for his advice and encouragement. Huge gratitude goes to the Industrial Research Limited and Victoria University of Wellington, New Zealand for full financial support of this study. Further acknowledgments extend to Liz Richardson, Deputy Dean (Equity) and Te Ropu Awhina for their additional financial support. I also extend my appreciation to Professor Tim Schulze (Department of Mathematics, University of Tennessee, USA) and Peter McGavin (Industrial Research Limited, New Zealand) for their collaboration and for allowing me to share their expertise in the research topic; and to my roommate, Nat Lund (PhD student in Physics), for sharing his experience. Special thanks must go to my parents for their upbringing and love. Finally, I am also thankful to my lovely wife and children for their sacrifices, wholehearted support, and understanding throughout the study. Without your love, I would have had no time to fulfill this dream. My additional thanking to my wife, Mele Katea Paea (PhD student in Management), for her continuous reading and checking of the whole thesis. 


\section{Contents}

1 Introduction 1

1.1 The main research goal and objectives . . . . . . . . . 2

1.2 The rationale for researching this topic . . . . . . . . 3

1.3 The significance of this study . . . . . . . . . . . . 4

1.4 Methodology ................. 6

1.5 The thesis structure ............... 7

2 Crystal Growth 9

2.1 The concept of crystal growth . . . . . . . . . . . 9

2.2 Crystal Structure . . . . . . . . . . . . . . . 11

2.3 Morphology of Crystals . . . . . . . . . . . . . 13

2.4 Theoretical Aspects of Crystal Growth . . . . . . . . . . . . 15

2.4.1 Thermodynamic Considerations . . . . . . . . . 15

2.4 .2 Equilibrium Shape . . . . . . . . . . . . 17

2.4.3 Equilibrium shape of a supported crystal . . . . . . 21

2.4 .4 Kinetic considerations . . . . . . . . . . . . 22

2.5 Nanostructure Growth . . . . . . . . . . . . . . . . . . . . 25

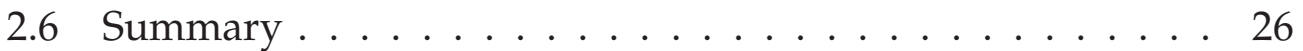

3 Kinetic Monte Carlo Methods 27

3.1 Background of the KMC . . . . . . . . . . . . . 28

3.2 Motivation: the time-scale problem . . . . . . . . . . . . . 29

3.3 Basic Principles of KMC . . . . . . . . . . . . . . 31 
3.3.1 Master Equation . . . . . . . . . . . . . . . 31

3.3.2 Solution of the Master Equation _. . . . . . . . 33

3.4 KMC Scheme for Crystal Growth . . . . . . . . . . . . 38

3.5 Implementation of the KMC Algorithm . . . . . . . . . . 40

3.6 Summary . . . . . . . . . . . . . . . . . . . . 43

4 Deposition and Growth KMC 45

4.1 Calculating the Hopping Rate . . . . . . . . . . . . 46

4.2 Deposition Process and Calculating the Deposition Rate . . . 48

4.3 Results and Discussion . . . . . . . . . . . . . . . . . . 51

4.3 .1 Multiple seeds. . . . . . . . . . . . . . 57

4.4 Summary . . . . . . . . . . . . . . . 62

5 Continuum-KMC Method 63

5.1 Introduction . . . . . . . . . . . . . . 63

5.2 Mass and heat transfer during crystal growth . . . . . . . 66

5.3 Mathematical formulation . . . . . . . . . . . . . 68

5.4 The discrete form of the governing equations . . . . . . 71

5.5 Calculating solidification and hopping rates . . . . . . . 74

5.6 Results and Discussion . . . . . . . . . . . . . . 76

5.6.1 Time step and physical parameters . . . . . . . 76

5.6 .2 Effect of Lewis number . . . . . . . . . . . 80

5.6 .3 Mass conservation . . . . . . . . . . . . . . 88

5.6 .4 Tip Velocity . . . . . . . . . . . . . . . . . . . . 91

5.6 .5 Anisotropy . . . . . . . . . . . . . . . 93

5.6 .6 Multicrystal growth . . . . . . . . . . . . 96

5.7 Dissolution . . . . . . . . . . . . . . . . . . . 100

5.7.1 Calculating the dissolution rate . . . . . . . . . . 101

5.7.2 Comparing Growth and Dissolution . . . . . . . . 102

5.8 Summary . . . . . . . . . . . . . . . . . . 105 
6 Diffusion and Growth KMC 107

6.1 Diffusion equation using the Monte Carlo method . . . . . . . . . . . . . . . . . 108

$6.2 \mathrm{KMC}$ algorithm . . . . . . . . . . . . . . . 109

6.2.1 Creating a single atom at the cell edges . . . . . . . 109

6.2 .2 Surface diffusion . . . . . . . . . . . . . 110

6.3 Results and Discussion . . . . . . . . . . . . . . 111

6.3.1 Multiple Seeds ............... . 113

6.3.2 Nature of crystal shape in two models . . . . . . . . . 114

6.4 Dissolution . . . . . . . . . . . . . . 116

6.4.1 Dissolution Process . . . . . . . . . . . 116

6.4.2 Comparing Growth and Dissolution . . . . . . . . . . 117

6.4 .3 Ostwald ripening . . . . . . . . . . . . 118

6.4.4 Two clusters of the same sizes dissolve and grow . . 120

6.5 Summary . . . . . . . . . . . . . . . . 121

7 Conclusion and Future Work 122

7.1 Conclusion . . . . . . . . . . . . . . . 122

7.2 Recommendations for future work . . . . . . . . . . 125

$\begin{array}{ll}\text { A Stochastic processes } & 126\end{array}$

B Markov Processes $\quad 128$

B.1 The Conditional Probability . . . . . . . . . . . . . . . 128

B.2 Markov Property . . . . . . . . . . . . . . . . . 129

$\begin{array}{ll}\text { C Derivation of the Master Equation } & 130\end{array}$

$\begin{array}{ll}\text { D Proof of Equation (3.9) } & 133\end{array}$

$\begin{array}{lll}\text { E Transition State Theory (TST) } & 135\end{array}$

$\begin{array}{lll}\text { F Supplementary Code } & 138\end{array}$ 


\section{Chapter 1}

\section{Introduction}

The purpose of nanoscience and nanotechnology is to understand, control, and manipulate objects of a few nano-meters in size (say, 1-100nm). These nano-objects are thus intermediate between single atoms and molecules and bulk matter. Their properties are often peculiar, being qualitatively different from those of their constituent parts (either atoms or molecules) and from those of macroscopic pieces of matter. In particular, nano-objects can present properties that vary dramatically with size. This opens the possibility of controlling these properties by precisely controlling their formation process.

Among nano-objects, nanoclusters or nanocrystals occupy a very important place, since they are the building blocks of nanoscience. Nanocrystals are aggregates of atoms or molecules of nanometric size, containing a number of constituent particles ranging in number from approximately 10 to $10^{6}$. Nanocrystals exhibit a variety of size- and shape-dependent physical and chemical properties that present a unique opportunity for creating materials with tailored characteristics [1]. Controlling the nanocrystal shape is a real challenge and more data is now needed to ascertain the general principles that determine this shape. The variation of crystal shape over different time scales is probably due to the fact that anisotropic materials are not in their thermodynamically stable state. 
A number of techniques (phase-fields method [2], level-set method [3], Monte Carlo method, adaptive mesh techniques [4], random walks with adaptive step sizes [5] and so forth) have been reported for modelling nanocrystal growth. The current study uses the kinetic Monte Carlo (KMC) algorithm to examine the growth morphology and structure of nanocrystals. It does so by examining the crystal growth in a gas of atoms and in an undercooled binary melt. The exploration included in the thesis is divided into three major parts, the Deposition and Growth KMC method, the Continuum-KMC Method, and the Diffusion and Growth KMC method. In the initial stage of the current study, the broad focus was to simply study a straight forward approach to developing a multiscale simulation method for the growth of nanocrystals in solution that couples a KMC description of the crystal relaxation process to solute reaction diffusion equations. In the course of the study, the findings reveal how the KMC technique has proven its significant contribution to the mathematical modelling of crystal growth. This has led to a better understanding of the atomistic process of crystal growth as one area of interest in applied mathematics, physics and materials science.

The purpose of this chapter is to provide an overview of the current study. It begins by considering the core goal and objectives. It then describes the rationale for researching this topic. The significance and methodology of this study are discussed, and the thesis structure is outlined.

\subsection{The main research goal and objectives}

Over the last decade the KMC method has been widely applied to study epitaxial crystal growth with much success $[6,7,8,9]$. However KMC has yet to be widely applied to solution phase crystal growth, especially for nanocrystal growth. Therefore, it is necessary to couple the theory of crystal relaxation with the mathematical understanding of solute diffusion fields. The main goal that guided the present research was: To develop a 
multiscale simulation method for the growth of nanocrystals in solution that couples a KMC description of the crystal relaxation process to solute reaction diffusion equations. This goal was developed to collect in-depth information for a better understanding of the phase crystal growth using the KMC method, one of the most powerful techniques for studying crystal growth. To achieve the main research goal, three research objectives were established:

1. to understand the process of a solidification event (the process of how an atom solidifies, that is, changing from a liquid atom into a solid atom) in order to extend the understanding of the suitability of the KMC algorithm for exploring the evolution and morphology of crystal structure;

2. to apply a numerical finite difference method by using an explicit discretization to solve the continuum model for heat and diffusion equations at the solid-liquid interface, and

3. to calculate the solidification and hopping rates and consider how the surface anisotropy effect could be included in our model.

\subsection{The rationale for researching this topic}

The presence of the computational challenge in this work was one of the primary factors that attracted the researcher's interest to this field. As in many areas of materials science, modern computational science is becoming a key contributor in the quest to quantitatively understand the molecular level mechanisms underlying the macroscopic phenomena in chemical processing. It is envisioned that this study will contribute to the rational design of crystal growth and to improve production strategies. Of particular relevance are hierarchical approaches that link the insights that modelling and simulation can provide across all relevant length and time 
scales [10]. The computer simulations allow the scientist to model events at the nanoscale that are difficult to observe experimentally. It is believed that some of the critical events in crystal growth occur at the nanoscale, which naturally motivates a simulation based approach. The simulation approach provides atomic scale precision data on the surface morphology and shows the detailed atom-based behaviours that are hard to be observed by experiments [11].

\subsection{The significance of this study}

Crystal growth involves a variety of research fields ranging from surface physics, crystallography, and material sciences to condensed matter physics. Although significant efforts have been made over the last few decades to predict the growth morphology of crystals, it remains a challenging task to date.

The various shapes of crystals are of great technological importance. This study will deal with the natural shapes of crystals, such as dendritic shapes. From a practical point of view, it is important to understand how crystals tend to grow in order to devise processes to control how they do so. For example, gemstones, for which nature has produced crystal morphologies and a degree of perfection, have yet to be duplicated by artificial processes. Another such example is the snowflake that decorates our world with beautiful different patterns whose artistic and scientific value alone is worth understanding. Also the morphological evolution and the growth mechanism provides crucial experimental input as a guide for fabrication of high-performance crystalline alloys for application in automobiles, aerospace and biomedicine [12]

Some particular facets are usable for industries such as the pharmaceutical industry. Crystals reveal a large variety of shapes, depending on the chemical composition, the structure of crystals, and the growth conditions. The shape of the crystals has a direct impact on the separation 
efficiency and the stability of crystalline chemicals, the bioavailability and the effective delivery of drugs. Knowledge of the growth habits and morphological properties of the molecular crystals is of crucial importance in understanding and exploiting many of their physico-chemical properties. Calculating the crystal growth morphology has diverse applications ranging from drug design [13] to explosives [14] and inverse gas chromatography data [15].

Controlling the morphology of nanoparticles is of key importance for exploiting their properties in several emerging technologies. For example, the applications such as electronic and optical devices [16], and biosensors [17]. These applications use optical properties of gold nanoparticles related to surface plasmon resonances, which depend strongly on the anisotropy of the particle shape, larger shapes produce greater plasmon losses [18]. Another example is the application of magnetic nanoparticles in data storage which is limited by superparamagnetism that precludes their use at room temperature [19]. One way to avoid this problem is to increase the magnetic anisotropy, by growing very anisotropic shapes for example [20]. In catalysis the shape of the catalyst particles often plays an important role [21, 22, 23]. The ability to precisely predict and control the morphology of nanoparticles that terminates with the desired crystallographic planes remains one of the main outstanding synthetic challenges.

The study of the diffusion, adsorption, deposition and solidification of atoms on a growing surface has been an active field in the past decade, because of both experimental and theoretical advances. Experiments can give detailed images of patterns formed on growing surfaces. An important challenge to the theoretical studies is the identification of dynamic processes controlling the pattern formation and overall surface morphology. This can be achieved by accurately modelling the atomic interactions, a thorough search for active-scale processes, and simulation of the growth on an experimental timescale to allow for detailed comparison with the experimental measurements. This study adopts a discrete, atomistic model 
in the spirit of the KMC simulations to examine the growth morphology and evolution of nanocrystals. It is apparent that the KMC algorithm has yet to be widely applied to solution phase crystal growth, especially to nanocrystal growth, in research. Taking into account this gap in the crystal growth literature, this study emphasises the value of the KMC technique in the understanding of nanocrystal growth. This makes the research of crystal growth in the current study different from that in the academic research of crystal growth processes in the past.

\subsection{Methodology}

This thesis is essentially a series of computer simulations with the aim of understanding nanocrystal growth. The two main components in this approach are: atomistic simulation and mathematical modelling.

The difficulty in understanding the crystal growth phenomena arises due to complex relationships between different processes, which take place on very large time and length scales, particularly in the case of industrial processes. The processes involved during the crystal growth are highly complex because they involve many-body interactions for accurate description of the problem. Nanocrystal growth is a nanoscale process and it is best understood in microscopic variables. That is, their properties have great potential in application and can be understood only with the knowledge of structures at the atomic level. The microscopic theory of crystal growth is a detailed understanding of the mechanisms of growth and of the effects arising from the change of physical quantities and material parameters. The main theoretical tools of microscopic theory are numerical simulations involving the application of the two basic methods: Molecular Dynamics (MD) and kinetic Monte Carlo (KMC). This study will make use of the KMC method. A broad overview of this well established technique can be found in $[24,25,26]$ but some of the relevant details will be outlined in Chapter 3. 
Computer simulations have become a useful part of mathematical modelling for a better understanding of the atomistic process of crystal growth. The modelling of crystal growth process in this study is via a mathematical model, which attempts to find analytical solutions to problems, and enable the prediction of the patterning of crystal growth from a set of convenient parameters and initial conditions. In the simulation process, if all relevant processes in the system have been identified, and the rate of each process has been estimated, the time evolution of the system can be described by a set of coupled rate equations (mathematical model) that can be solved using a Monte Carlo approach. This is the so-called kinetic Monte Carlo procedure. In conclusion, the advantage of modelling is that it often allows us to see a path through the complexity inherent in nanoscience and nanotechnology.

\subsection{The thesis structure}

This thesis comprises seven chapters, including this introductory chapter. Chapter Two presents a review of the concept of crystal growth including the following: crystal structure of a Face Centred Cubic (FCC) crystal, morphology of crystal structure, theoretical aspects of crystal growth. Chapter Three explains the kinetic Monte Carlo (KMC) methods and studies relevant to the current study. Chapter Four describes the details of examining the crystal growth in the situation where there is a nanocrystal sitting in a gas of atoms and introduces deposition events. Chapter Four also presents the first part of how the KMC code was reviewed and used as a guide for the entire computation in the current study.

Chapter Five describes the use of the KMC algorithm to examine the growth morphology and structure of the nanocrystals in an undercooled binary melt. The main research methods provide an explanation of how we solved the continuum model for heat and solute equations at the solidliquid interface. Chapter Five presents the research findings, and Chapter 
Six explains the case where the KMC algorithms in Chapter Four and Five are extended to model diffusion in the solute. Chapter Seven concludes this thesis with an overall review of the research's main goals and objectives in response to the general findings, reflections on the current study, and suggestions for future action and research. The digital diagram of 'crystal growth in 3-dimensions' can be viewed on a DVD attached to this thesis. 


\section{Chapter 2}

\section{Crystal Growth}

This chapter aims to provide a background understanding of crystal growth appropriate for the current study. The chapter begins with what theorists and researchers say about the concept of crystal growth and in order to lay the foundation for subsequent chapters, it also describes the evolution and morphology of crystal growth under equilibrium and nonequilibrium conditions. It then carries on to give a general discussion of the structure of the Face Centred Cubic (FCC) crystal, and the theoretical aspects of crystal growth. The chapter concludes with an explanation of nanostructure growth.

\subsection{The concept of crystal growth}

The topic of crystal growth has played a very important role in modern technology and much research has been published on the theory of crystal growth. Interest in studying the concept of crystal growth can be traced back to studies in the 17th century. In his book Crystal Growth, Morphology and Perfection, Sunagawa [27] recalled the concepts of crystal growth from the 17th to the 20th century. In the 17th century, Sunagawa [27] explained, the growth rates of crystal related to crystallographic direction. This means that the growth rates of crystals are different depending on 
the different crystal faces (Steno, 1638 - 1687), for example, the hexagonal prismatic forms can be seen by six faces at the tip, and sometimes show tapering prismatic or platy forms. Hence the natural morphology of crystals arises in part from crystalline anisotropy, the fact that crystallographic properties depend on orientation. These concepts of crystal growth and growth rate anisotropy provide a basis for the present-day science of crystal growth including the current study.

In the 18th century, the study of crystal growth was moving from the crystallographic direction to how it grows in aqueous solution or in water [27]. Hooke [28] found that the shape of crystals different as they grew on the tip of the string immersed in aqueous solution and at the bottom of a beaker but both shapes bounded by the same faces. In relation to the growth of crystals in the water, Hooke's [28] and Holden and Marison's [29] findings on crystal growth are similar in nature. Holden and Marison [29] noted that in the growing of alum crystal, alum such as sulphate and potassium sulphate diffuse through the water and when they reach the surface of the crystal, they join with each other and with some of the water. They adopt positions on the surface that are forced on them by the kind of orderliness confronting them. Settling into those positions, they extend the orderliness outward, and thus the crystal grows.

The idea of crystal growth in the 19th century was centred around the understanding of crystals as unit cells [27] and much of the work on crystal growth in the 20th century has been largely based on the growth on crystal surfaces with both stable and unstable growth scenarios $[29,30,31,32,33$, $24,34]$. This has now advanced to a state to be understood from a unique viewpoint, the atomistic process of crystal growth. It is now possible to explain at the atomistic level why and how the same crystal can take a variety of forms, from dendritic (branching like a tree), hopper (the edges of hoppered crystal are fully developed, but the interior spaces are not filled in), to polyhedral forms, and why different crystal species exhibit different characteristic form. How our understanding of morphology and 
growth mechanism of crystals has developed since the time of Steno has recently been summarized by Sunagawa [27], Holden and Morrison [29], and Byrappa and Ohachi [35].

Kittel [31] noted that when a crystal grows in a constant environment, the form develops as if identical building blocks (atoms, molecules, or ions) were arranged in an orderly repeating pattern extending in all three spatial dimensions. For better understanding of surface growth, the current study recalls the two basic and related questions: what is the growth mode and what is the growth kinetics? The growth kinetics and the different modes of crystal growth are well described in an article by Levi and Kotrla [24] in their review of crystal growth simulation in relation to KMC methods. This study is concentrated on three-dimensional growth, in which many crystal layers grow at the same time, which is in contrast to the two-dimensional growth mode. In this mode, the atoms are depositing at relatively high rates that cause the surface to form hillocks and cavities and the mode of growth can change with the strength of the disequilibrium.

\subsection{Crystal Structure}

In order to understand the crystal growth, it is useful to discuss the structure of crystals. A crystal structure is defined as the particular repeating arrangement of atoms (molecules or ions) throughout a crystal. It is composed of a pattern, a set of atoms arranged in a particular way, and a lattice exhibiting long-range order and symmetry. This section examines the structure of a Face Centred Cubic (FCC) crystal, central to the current study.

Crystals are solid materials having regular arrangement of atoms, molecules, or ions. Crystal forms are determined by structure and the factors involved in growth. The structure of all crystals can be described in terms of a lattice, with a group of atoms attached repeating periodically to ev- 
ery lattice point in three dimensions. That is, these lattice points are of fundamental importance in describing crystals for they may be the positions occupied by individual atoms in crystals or they may be points about which several atoms are clustered. An important characteristic of a space lattice is that every point has identical surroundings: the grouping of lattice points about any given point is identical to the grouping about any other point in the lattice.

The lattice is defined by three fundamental translation vectors $\mathbf{a}_{1}, \mathbf{a}_{2}$, $\mathbf{a}_{3}$ such that the atomic arrangement looks the same in every respect when viewed from the point $\mathbf{r}$. When viewed from the point $\mathbf{r}^{\prime}=\mathbf{r}+\mathbf{x}_{i j k}$ where $\mathbf{x}_{i j k}=i \mathbf{a}_{1}+j \mathbf{a}_{2}+k \mathbf{a}_{3}$ is the integer combinations of three basis vectors for the FCC lattice. The primitive translation vectors $\mathbf{a}_{1}, \mathbf{a}_{2}$, and $\mathbf{a}_{3}$ connect the lattice point at the origin with lattice points at the face centers. The primitive translation vectors of the FCC lattice of Figure 2.1 are

$$
\mathbf{a}_{1}=a\left(\frac{\widehat{\mathbf{i}}+\widehat{\mathbf{j}}}{\sqrt{2}}\right) ; \quad \mathbf{a}_{2}=a\left(\frac{\widehat{\mathbf{i}}+\widehat{\mathbf{k}}}{\sqrt{2}}\right) ; \quad \mathbf{a}_{\mathbf{3}}=a\left(\frac{\widehat{\mathbf{k}}+\widehat{\mathbf{j}}}{\sqrt{2}}\right),
$$

that are themselves formed by combinations of the equilibrium distance between two atoms $a$ and the Cartesian unit vectors $\widehat{i}, \widehat{j}$ and $\widehat{k}$. The primitive translation vectors $\mathbf{a}_{1}, \mathbf{a}_{2}$, and $\mathbf{a}_{3}$ connect the lattice point at the origin with lattice points at the face centers. The black circles are the lattice points at the corners and the red circles are the lattice points at the face centers. The angles between the axes are $60^{\circ}$. Upon scaling, $\left\{\mathbf{a}_{\mathbf{1}}, \mathbf{a}_{\mathbf{2}}, \mathbf{a}_{\mathbf{3}}\right\}$ become unit vectors and it is useful to expand this set to the twelve vectors

$$
\{\mathbf{e}\}_{i=1}^{12}=\left\{ \pm \mathbf{a}_{\mathbf{1}}, \pm \mathbf{a}_{\mathbf{2}}, \pm \mathbf{a}_{\mathbf{3}}, \pm\left(\mathbf{a}_{\mathbf{1}}-\mathbf{a}_{\mathbf{2}}\right), \pm\left(\mathbf{a}_{\mathbf{2}}-\mathbf{a}_{\mathbf{3}}\right), \pm\left(\mathbf{a}_{\mathbf{3}}-\mathbf{a}_{\mathbf{1}}\right)\right\}
$$

that point to the nearest neighbours of a given lattice site. Most solids are crystalline, meaning that their particles (atoms, molecules, or ions) are arranged in a repetitive lattice structure extending over significant distances in atomic terms. In this context atoms may be regarded as spheres of di- 
ameter 2 to 6 Angstroms ( 1 Angstrom $=10^{-10}$ meters).

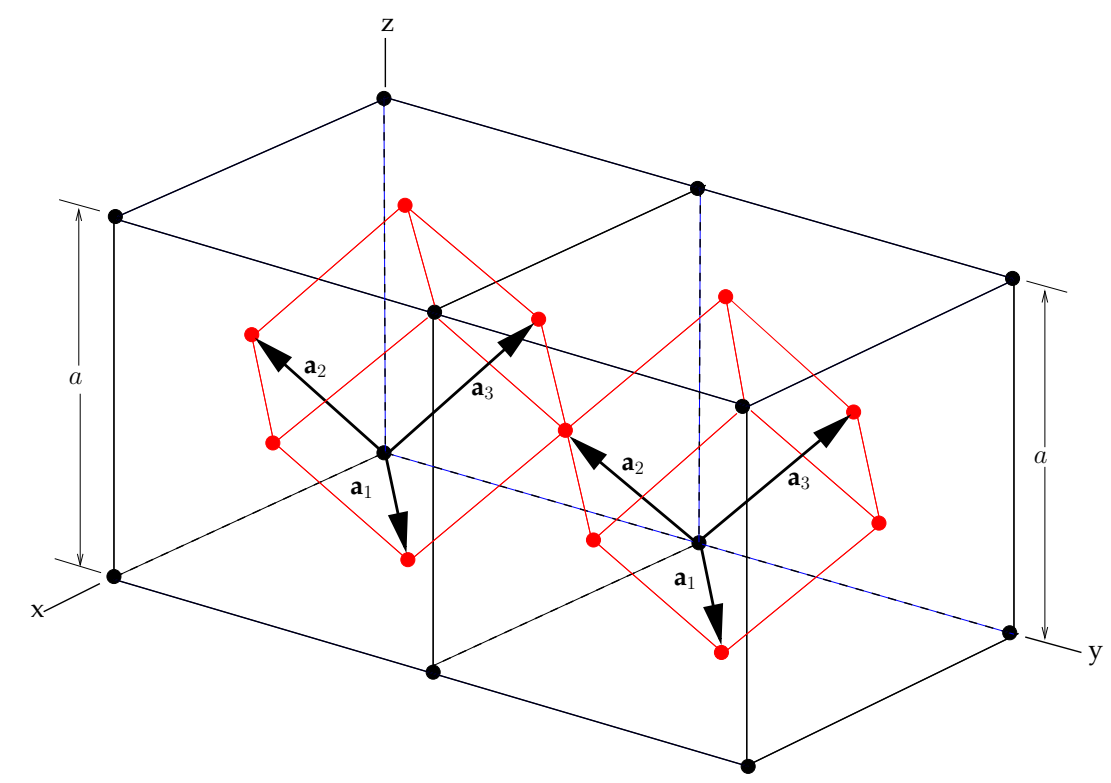

Figure 2.1: The rhombohedral primitive cell of the two face-centered cubic unit cells. The primitive translation vectors $\mathbf{a}_{1}, \mathbf{a}_{2}$, and $\mathbf{a}_{3}$ connect the lattice point at the origin with lattice points at the face centers. The black circles are the lattice points at the corners and the red circles are the lattice points at the face centers. The angles between the axes are $60^{\circ}$.

\subsection{Morphology of Crystals}

Since the morphology of crystal structure is one of the objectives of the current study, it is therefore important to discuss the morphology of crystals. Our intention is to present systematically the fundamental concepts that allow us to analyze the factors that determine the various forms of crystals. Crystals are polyhedral objects which can have flat surfaces of more than one kind. These surfaces are called faces and each kind is characterized by a distinct arrangement of atoms, molecules or ions, which leads to a specific growth rate. The faces that have identical arrangements of atoms grow at the same velocity, while the faces that have different arrangements 
of atoms grow at different rates. This difference in growth rates leads to morphological evolution of the crystal. Sometimes the crystalline shapes are more complicated figures, dendritic, spherulitic, or fractal, but they all are polyhedral, that is, figures bounded by a finite number of plane faces.

The morphology of a crystal is determined by the crystal structure (the internal factors), the crystal growth conditions and the process of that growth (the external factors) [27]. The internal factors will lead to equilibrium crystal forms with minimum total surface free energy. Different external factors normally influence crystal forms and force the crystal to deviate from the equilibrium form to develop into various morphologies. The crystal shape is derived from the competition of these internal and external factors, and its growth is related to the growth kinetics, such as interface property, capillarity, and heat and mass transfer [36].

Growth and dissolution processes of crystals uniquely take place on the surfaces of a crystal, that is on the solid-liquid (ambient phase) interface. The processes depend on the structure of the interface, whether it is rough or smooth, the growth mechanism, and thus the relations between the driving force and growth rate are different. The growth rate and the driving force are related as follows:

$$
R=A(\Delta \mu / k T)^{2}
$$

where $A$ is a constant, $\Delta \mu$ is the difference of chemical potentials between the two phases, $k$ is the Boltzmann constant, and $T$ is the absolute temperature. The expression of the driving force in terms of the generalized driving force is $\Delta \mu / k T$.

The growth of interface is different depending on crystallographic directions (growth rates), which are related to the crystal structure. This leads to different shapes of crystal where the interface structure transforms from smooth to rough with increasing growth temperature (thermodynamic roughening transition) and driving force (kinetic roughening transition) [35]. Summarizing these, the relations among the different shapes 
is shown in Figure 2.2, which represents only a section of events but can be utilized as a standard [27]. Figure 2.2 shows the growth rate $R$ against the driving force $\Delta \mu / k T$ for three models of growth mechanism. This figure illustrates the following two points:

1. the interface becomes rougher, as the driving force increases, and

2. two bending points (critical points) appearing at $\Delta \mu / k T^{*}$ and $\Delta \mu / k T^{* *}$ are the points where the predominant growth mechanism changes [27]. The relation of the curves $R$ against the $\Delta \mu / k T$ is expected to be different for the three models of growth mechanisms. The growth mechanism will be adhesive-type above $\Delta \mu / k T^{* *}$ and the interface becomes rough. Below $\Delta \mu / k T^{*}$ the growth will be principally controlled by the spiral growth mechanism and the interface will be smooth. In between $\Delta \mu / k T^{*}$ and $\Delta \mu / k T^{* *}$, the growth mechanism will be principally two-dimensional nucleation and the interface is still smooth.

Depending on phases, materials, and sizes, the positions of $\Delta \mu / k T^{*}$ and $\Delta \mu / k T^{* *}$ may change. In the following sections, we discuss some basic ideas on how to control the shapes of particles by controlling their growth.

\subsection{Theoretical Aspects of Crystal Growth}

\subsubsection{Thermodynamic Considerations}

The equilibrium morphology of crystals, as determined by thermodynamics, can be obtained by minimizing the total free surface energy of the crystal at a constant volume and temperature [37, 38]. For isotropic surface free energies (as for a liquid), the crystal morphology will be spherical in shape and the chemical potential constant everywhere on the surface. The 


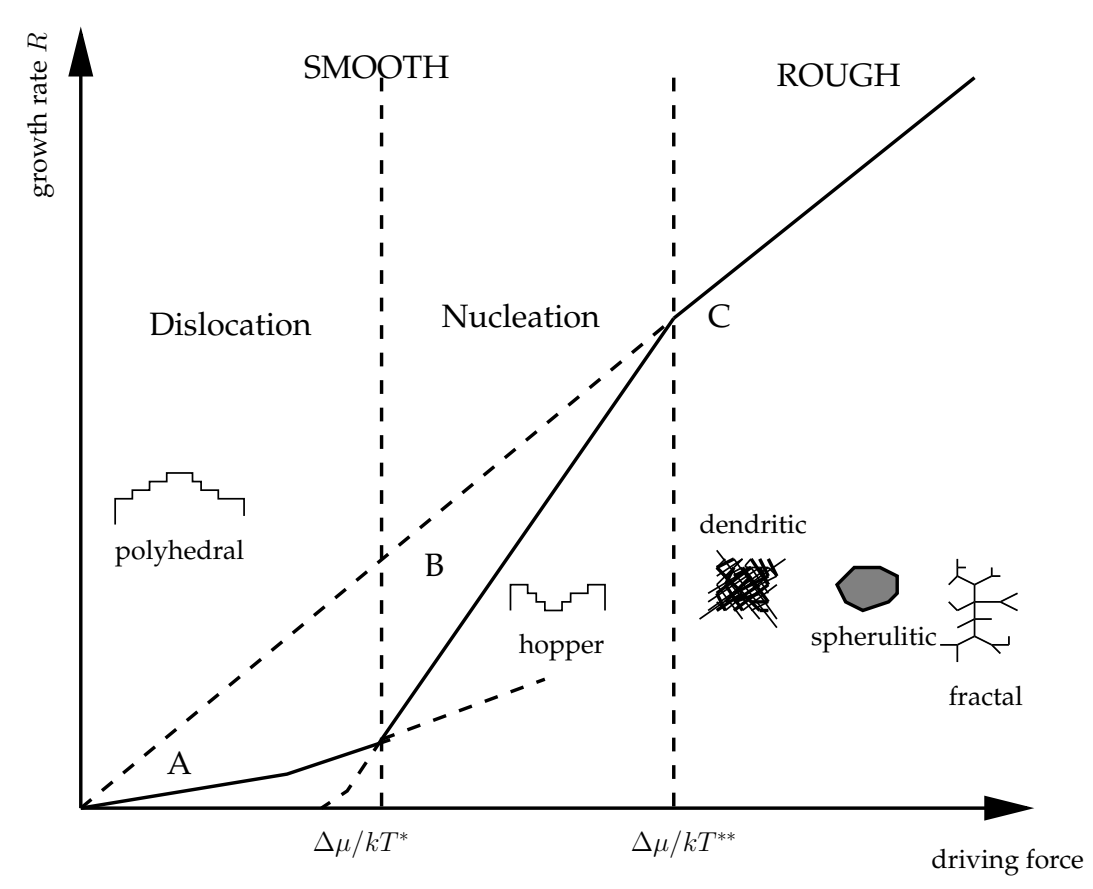

Figure 2.2: Schematic diagram showing the growth rate $\mathrm{R}$ against the driving force $\Delta \mu / k T$, morphologies of single crystals (polyhedral, hopper, dendritic) and polycrystalline aggregates (spherulitic and fractal) in relation to growth rate $\mathrm{R}$, driving force, interface roughness (smooth and rough) and growth mechanisms. Curve A represents the spiral growth, curve $B$ the two-dimensional nucleation growth, and curve $C$ the adhesive-type growth mechanisms.

calculation of the chemical potential is given by [37]

$$
\mu=\mu_{0}-\frac{V}{N}\left[\frac{\partial}{\partial x}\left(\frac{\partial \phi}{\partial z_{x}}\right)+\frac{\partial}{\partial y}\left(\frac{\partial \phi}{\partial z_{y}}\right)\right]
$$

where $\mu_{0}$ is the chemical potential inside the crystal, $V$ is the volume of the crystal, $N$ is the number of atoms in the crystal, and $z_{x}=\frac{\partial z}{\partial x}$ and $z_{y}=\frac{\partial z}{\partial y}$ are the partial derivatives of the height, $z$, over the coordinates of the $(x, y)$ plane respectively. It is convenient for a closed surface if the surface is cut into a few pieces and different projection planes are used for the various pieces (e.g. $x y, y z$, and $z x$, each twice). For a given piece, the projected 
surface free energy $\phi$ is written as $\phi\left(z_{x}, z_{y}\right)=\sigma\left(z_{x}, z_{y}\right) \sqrt{1+z_{x}^{2}+z_{y}^{2}}$. Thus, the surface free energy, $\mathcal{F}_{\text {surf }}$, is defined as follows:

$$
\mathcal{F}_{\text {surf }}=\iint \sigma\left(z_{x}, z_{y}\right) d S
$$

where $\sigma\left(z_{x}, z_{y}\right)$ is the local surface tension of the partial derivatives $z_{x}=$ $\partial z / \partial x$ and $z_{y}=\partial z / \partial y$. The integration is made over the surface and $d S$ is the surface element. The constant chemical potential implies that the surface free energy (given by Equation (2.2)) is at a minimum at equilibrium. Equivalently, if one takes an atom from a position where $\mu=\mu_{1}$ to a position where $\mu=\mu_{2}$, the corresponding surface free energy, $\Delta \mathcal{F}$, at a constant temperature and volume is given by $\Delta \mathcal{F}=\mu_{2}-\mu_{1}=0$.

\subsubsection{Equilibrium Shape}

In particular, the equilibrium shape of any macroscopic object is determined by the requirement that the chemical potential $\mu$ be a constant. The crystal surface can consequently be described by Equation (2.1), provided that the function $\mathcal{F}\left(z_{x}, z_{y}\right)$ or $\phi\left(z_{x}, z_{y}\right)$ are known. Assuming, that the units are selected in such a way that the molar volume $v=V / N=1$, and $\mu_{0}=0$, the equation for the crystal surface can be written as

$$
\frac{\partial}{\partial x}\left(\frac{\partial \phi}{\partial z_{x}}\right)+\frac{\partial}{\partial y}\left(\frac{\partial \phi}{\partial z_{y}}\right)=-\mu=\text { Constant. }
$$

Equation (2.3) can only be applied to crystal morphologies if the derivatives of $\mu$ exist. This will be the case for rounded crystals since $\mu$ for faceted crystals is only defined in a discrete set of orientations corresponding to the facets in the equilibrium morphology (see Figure 2.3). For a faceted crystal (Figure 2.3), the surface free energy can be written as a sum of the 
contributions from the various facets

$$
\mathcal{F}_{\text {surf }}=\sum_{f} \sigma_{f} A_{f}
$$

where $\sigma_{f}$ is the value of $\sigma(\mathbf{n})$ at $\mathbf{n}=\mathbf{n}_{f}$, and $A_{f}$ is the area of the corresponding facet. The equilibrium morphology can be found by minimising $\mathcal{F}_{\text {surf }}$ at a fixed volume $V=\frac{1}{2} \sum_{f} A_{f} h_{f}$, where $h_{f}=\max _{\mathbf{R}}\left\{\mathbf{R} \cdot \mathbf{n}_{f}\right\}$, and $\mathbf{R}$ being any point on the crystal surface. A constrained minimisation with a Lagrange multiplier $\lambda$ [39] can be performed to enforce the fixed volume condition

$$
\delta\left(\lambda V+\mathcal{F}_{\text {surf } f}\right)=\sum_{f}\left(\frac{\lambda}{2} h_{f}+\sigma_{f}\right) \delta A_{f}=0 .
$$

This minimization yields

$$
\frac{\sigma_{f}}{h_{f}}=-\frac{\lambda}{2}
$$

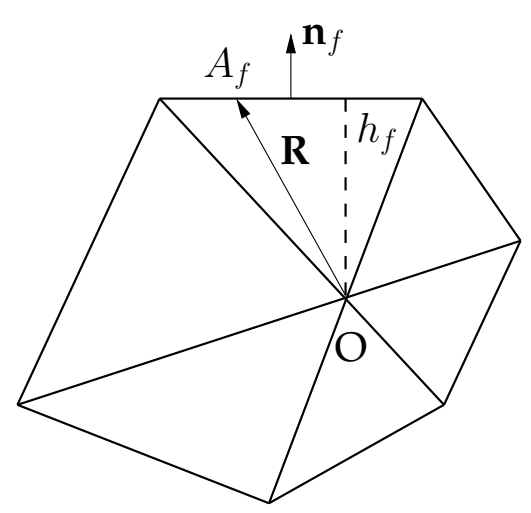

Figure 2.3: The equilibrium shape of a two-dimensional faceted crystal. The 'area' of the $f$-th facet is $A_{f}$ and $h_{f}=\mathbf{R} \cdot \mathbf{n}_{f}$ is the distance from the center of symmetry with $\mathbf{R}$ being any point on the crystal surface.

For each facet, the ratio of the surface energy to the distance $h_{f}$ from 
the origin is a constant. The expression for the set of $\left\{h_{f}\right\}$ gives the equilibrium morphology. At this stage, $\lambda$ is still unknown and it can be calculated by rewriting the variation in free energy at fixed volume as $\delta \mathcal{F}_{\text {surf }}=\mu \delta N$, where $\delta N$ is the variation in particle quantity $N$. According to Equation (2.4), $\delta\left(\lambda V+\mathcal{F}_{\text {surf }}\right)=0$, therefore

$$
\delta \mathcal{F}_{\text {surf }}=\mu \delta N=-\delta V \lambda=-v \delta N \lambda,
$$

with $v=V / N$ the volume per particle. It follows that $\lambda=-\frac{\mu}{v}$, where the Lagrange multiplier $\lambda$ appears to be proportional to the chemical potential, which is thus responsible for setting the overall size of the crystal. So Equation (2.5) can be written in the form

$$
\mu=\frac{2 v \sigma_{f}}{h_{f}} .
$$

In crystalline solids the surface energy is anisotropic and the energyminimizing shape is found using the limiting planes of the lowest possible surface energy. Wulff found this solution for the equilibrium shape of a crystal which is expressed by Wulff's theorem

$$
\frac{\gamma_{i}}{h_{i}}=\text { constant }
$$

where $\gamma_{i}$ is the surface energy and $h_{i}$ is the central distance to the facet of index $i$. An example of equilibrium morphologies for FCC structure are the truncated octahedron containing eight hexagonal (111) facets and six square (100) facets. For a BCC structure it is a rhombic-dodecahedron presenting twelve lozenge (110) faces respectively, as shown in Figure 2.4. These regular polyhedron shapes are only valid at $0 \mathrm{~K}$ where the surface energy anisotropy is maximal. At high temperatures, the surface energy anisotropy decreases, and the crystal equilibrium morphology is more round [40] and eventually becomes completely spherical at the melting point $[37,38]$. In addition to the above methodology, a geometric construc- 
tion, namely the Wulff theorem [37], can be used to find the equilibrium crystal morphology.

(a). FCC truncated octahedron

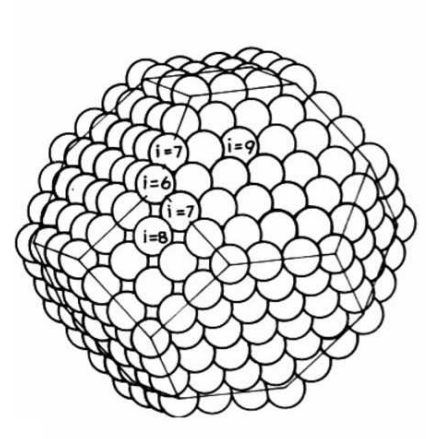

(b). BCC rhombic dodecahedron

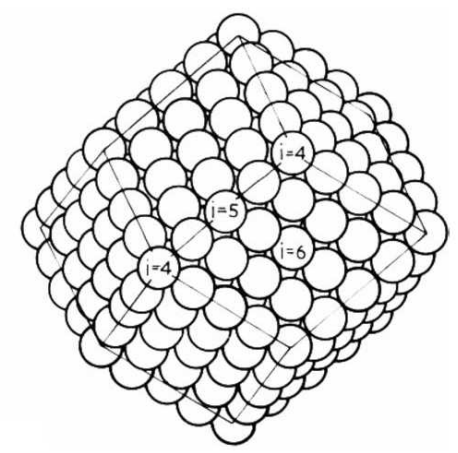

Figure 2.4: Equilibrium shape at $0 \mathrm{~K}$ of a (a) FCC truncated octahedron and a (b) BCC rhombic dodecahedron (from Henry [38]).

In this study we are interested in particles with nanometer dimensions. This emphasis on nanostructures brings into question the validity of the Wulff theorem in this size range. In order to examine its validity for studying nanostructures, several factors that can change the equilibrium shape when scaling down from macro to nanoscale should be considered. These include:

- both the surface energy and the surface stress increase [41];

- different structures such as icosahedral, as an example, can become more stable [42], and

- the proportion of edge atoms of different facets can no longer be neglected. Even if the crystal structure remains bulk-like, the equilibrium shape can change. This can be seen using the simple first neighbour two-body interaction [43]. For example, consider a Wulff shape 
limited by (111) and (100) facets with $n$ and $m$ respectively the number of atoms along the edges of these facets. The anisotropy of the surface energy is given by [44]

$$
\frac{\gamma_{(100)}}{\gamma_{(111)}}=\sqrt{3} \cdot \frac{n+m}{n+2 m} .
$$

For a given number of atoms one can calculate the value of $n / m$ that minimises the surface energy. In a macroscopic crystal, $n=m$, and the anisotropy factor is $2 / \sqrt{3} \approx 1.15$. In a nanometer sized structure, the (100) facets disappear which means that $m \rightarrow 0$; consequently $n / m \rightarrow \infty$. Under these conditions the anisotropy factor tends to $\sqrt{3}$. Applying an anisotropic factor of $\sqrt{3}$ and using the Wulff construction [37], an octahedral shape is obtained. This is indeed the morphology for a nanostructure in which (100) facets are absent.

\subsubsection{Equilibrium shape of a supported crystal}

The above discussion is valid for crystal growth in free space, in which the current study is undertaken. However, nanoparticles are usually grown on supports and the equilibrium shape of a supported crystal is expressed by the Wulff-Kaischew theorem:

$$
\frac{\Delta h}{h_{i}}=\frac{E_{a d h}}{\gamma_{i}},
$$

with $\Delta h$ being the amount by which the crystal shape is truncated. $h_{i}$ and $\gamma_{i}$ are the central distance to the facet parallel to the interface and the corresponding surface energy. $E_{a d h}$ is the work of adhesion which equals the work necessary to separate the crystal from the support by an infinite distance (see Figure 2.5). It is useful to combine the Wulff-Kaischew theorem (Equation 2.7) with Young's equation for mechanical equilibrium. This relationship provides a means of using the adhesion energy to determine whether or not a supported crystal will wet a surface. More details of 


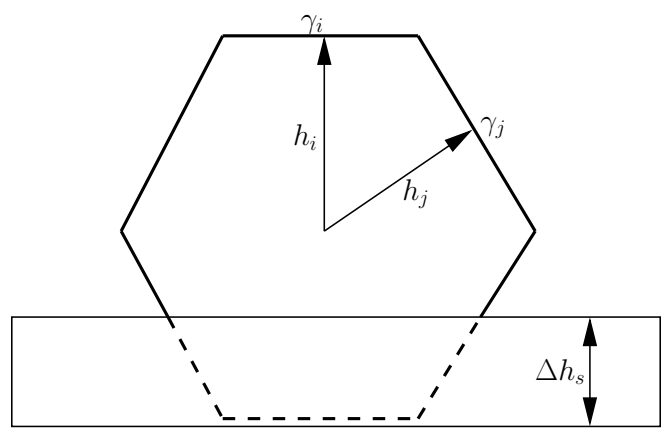

Figure 2.5: Schematic representation of the equilibrium shape of a supported polyhedron crystal. The shape of the free crystal is truncated at the interface by an amount $\Delta h_{s}$ which is proportional to the adhesion energy. The $h^{\prime} s$ represent the distance from the centre of the crystal to the different facets and $\gamma^{\prime} s$ illustrate the surface free energies.

the equilibrium shape of a supported crystal and the relationship between the Young equation and the Wulff -Kaischew theorem are described by Pimpinelli et al. [37] and Henry [38].

\subsubsection{Kinetic considerations}

In the preceding sections, crystal morphology and growth were discussed at thermodynamic equilibrium. However, in practice, crystal growth rarely occurs under equilibrium conditions. This happens due to the supersaturation, $S$, which is the ratio of the pressure around the growing crystal and the equilibrium pressure at the same temperature when $S$ is typically larger than one. In general the shape of the crystal depends on the growth rate of different faces as shown in Figure 2.6. Crystal faces are classified into three different types:

1. flat or F-faces which are parallel to at least two dense atomic rows;

2. stepped or $S$-faces which are parallel to at least one dense atomic row, and

3. kinked or $K$-faces which are not parallel to any dense atomic rows. 


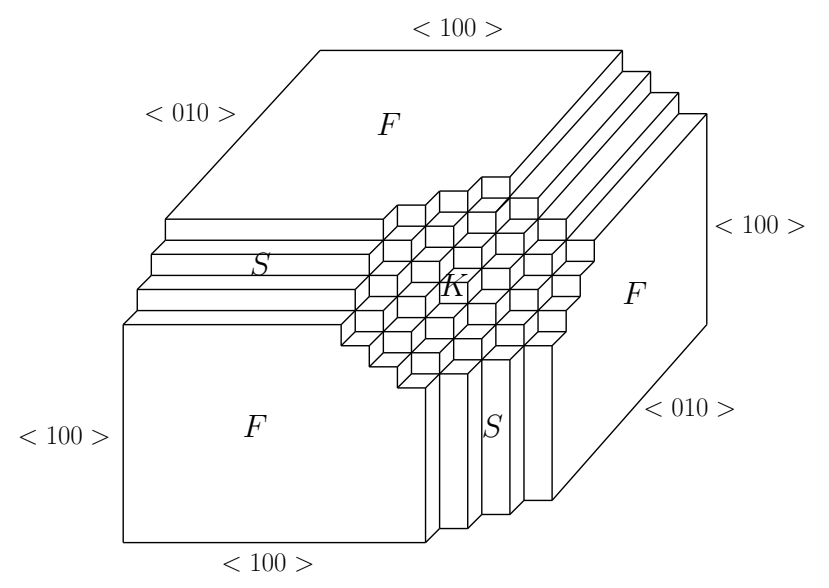

Figure 2.6: Schematic illustration of the various kinds of facets $S, F$ and $K$ on a growing crystal.

A $K$-face corresponds to a rough interface, an $F$-face to a smooth interface, and an $S$-face to a face having an intermediate nature between that of the $K$ and $F$ faces. Further, an $F$-face is mostly atomically flat. Growth on this face is thus only possible if (i) the supersaturation is large enough or (ii) the growth temperature exceeds the roughening temperature. The roughening temperature indicates the threshold above which surface roughening of an F-face occurs. The $S$ and $K$ faces, on the other hand, are atomically rough, as shown in Figure 2.6, and grow spontaneously. A $K$-face grows by the adhesive-type growth mechanism, an $F$ face grows either by a layer-by-layer or a spiral growth mechanism, and an $S$-face appears by the pilling up of growth layers advancing on the neighbouring $F$-face. Therefore, an $F$-face develops to a large size in order to control crystal habit in a real crystal (the term crystal habit is sometimes used in a broader sense to describe the characteristic forms shown by polycrystalline aggregates, such as spherulitic, botryoidal, or reniform) [27], the $K$-face will disappear from the crystal surface, and the $S$-face will be characterized by striations only, if it appears on a crystal.

The growth rate of a crystal face depends primarily on the supersaturation but also on other factors that we will discuss later. The $F$-faces 
clearly grow much slower than the $S$-faces and $K$-faces. Hence, a growing crystal will be limited only by $F$-faces that correspond to the lowest surface energy. However, the kinetically limited shape is not necessarily the equilibrium shape [37], for instance, a FCC structure, assuming that the growing crystal is limited, at a given time interval, by (100) and (111) facets. The (111) facets seem to grow more slowly than the (100) facets for particular conditions. The final shape of the crystal will end up as an octahedron.

The existence of facetted (or anisotropic) growth morphologies can primarily be attributed to the anisotropy in the flow of material to the different facets. Several factors can contribute to this source of anisotropy:

- Deposition flux and surface diffusion: In the case of vapour growth on a substrate, if the main flux comes from diffusion on the substrate, and if the surface diffusion is anisotropic, the growth shape will also be anisotropic;

- Presence of defects: Defects also lead to the growth of anisotropic crystals. For example, circular forms occur due to the presence of screw dislocations that increase the growth rate in one direction;

- Presence of impurities: Impurities can significantly influence the growth shape of a crystal. Impurity ions absorb preferentially on the (111) faces and drastically reduce the growth rate in this direction;

- Twinning: Twinning generates reentrant corners that are repeatable growth sites. Twinned crystals are elongated in one direction or flat [45]. Successive twinning in a $<111>$ direction gives rise to platelet triangular FCC nanocrystals [46], and

- Coalescence: If two growing crystals touch one another, they will produce an anisotropic form that will persist unless the temperature is elevated so as to increase surface diffusion to the extent that matter is redistributed between the different facets [45]. 


\subsection{Nanostructure Growth}

Studying the equilibrium morphology and size of nanostructures and crystals is an essential step to understand how these are influenced by various factors. Examples mentioned in the previous section include surface diffusion, defects, impurities and so forth. Naturally, precise control of these factors can eventually satisfy the need to tailor the morphology and size of nanostructures. However, the critical significant event is first determining the processes that contribute to the growth of the equilibrium or nonequilibrium morphologies in crystal growth. The current study is focused into three major parts:

1. In Chapter 4, we consider a supersaturated vapour of atoms condensing into a cluster. Deposition is considered to be equally likely to occur at any part of the crystal surface. As we consider an FCC crystal structure, the vapour could be considered to be that of an inert gas, or an FCC metal.

2. In Chapter 5, we consider the growth of a dendrite in a cooling binary alloy. The model allows for both phase change and exchange between liquid and solid atoms (that is, solidification processes) on the surface of the crystal and is coupled to a continuum model for heat and solute transport.

3. In Chapter 6, we consider growth from a supersaturated solution. Diffusion in the solute and surface diffusion on the crystal surface are handled completely by the KMC algorithm without the use of a continuum description of the solute.

The relevant processes that lead to nanostructure formation in the above situations can therefore be addressed as follows:

a. deposition of atoms (now termed adatoms) on the surface of the cluster; 
b. surface diffusion of an adatom ;

c. surface diffusion of an adatom along the edge;

d. dissociation of an adatom from the surface of the cluster;

e. melting of an adatom;

f. solidification of an adatom;

g. desorption from the surface, and

h. single atom diffusion.

All of these processes have a certain energy barrier before can occur and they have different time scales. The difference in the time scales can be problematic when simulation studies for nanostructure and surface growth are conducted. An overview of the time scales related to the current study is given in the next Chapter.

\subsection{Summary}

In general, the overview of crystal growth presented in this chapter builds a picture of crystal growth processes which are appropriate for the current study. What also emerges is that all research on crystal growth processes is based on the atomic construction which is the most commonly encountered state of solid materials. The present study therefore incorporates an investigation of the crystal growth processes for the purpose of understanding the structure and evolution of face centred cubic (FCC) crystals. In the present study, we have chosen to examine the surface growth with both stable and unstable growth scenarios as is commonly discussed in relevant literature. The study also attempts to investigate the effects of surface anisotropy, and the basis for the atomistic simulation process in this study involves the KMC algorithm. 


\section{Chapter 3}

\section{Kinetic Monte Carlo Methods}

Kinetic Monte Carlo (KMC) methods are used to model non-equilibrium systems using Monte Carlo (MC) simulation techniques. A typical example of such a problem is in crystal growth, where the adatom configuration on the surface is a complex function of temperature, flux, and microscopic energetics. To understand the analytic solution of the dynamics of the crystal morphology, KMC methods have been developed that simulate crystal growth by applying the physical rules to a simplified model of the system. From a comparison of these models with experimental results, insight into crystal growth process can be obtained.

This chapter describes the modelling methodologies used in this study. The background of KMC algorithms and their importance to the current study are discussed. Next, the motivation for using the KMC method with the time-scale problem is presented and the basic principles of KMC are explained. The explanation of the KMC scheme for crystal growth and the implementation of the KMC algorithm are discussed. The theoretical aspects of crystal growth and nanostructure growth are also discussed. 


\subsection{Background of the KMC}

Monte Carlo (MC) algorithms have been used in a wide variety of science and non-science disciplines, including materials science, nuclear physics, economics, and traffic flow. Monte Carlo refers to a broad class of algorithms that solve problems through the use of random numbers [47, 48]. They first emerged in the late 1940s and 1950s as electronic computers came into use [49], and the name reflects the statistical properties of random events which can be seen in the gaming in the casinos of Monte Carlo. The most widespread application of MC algorithm in materials science is determining the equilibrium structure or thermodynamic properties of materials [50]. These applications are based on the idea that the probability of different configurations occurring in a system depends both on the relative energies of those configurations and on their relative abundance in the phase space.

In early 1970s researchers began to develop a different kind of Monte Carlo algorithms for systems evolving dynamically from state to state. The earliest application of this approach for an atomistic system of crystal growth and evolution using probabilistic rules to govern deposition, diffusion and other transition processes was first adopted by Abraham and White [51] and Gilmer and Bennema [52]. Over the next 20 years, there were developments and applications in this area (for example, see [53, 54, $55,56,57,58])$. In the 1990s the terminology for this approach settled in as kinetic Monte Carlo, though the early and even some recent papers typically do not use this term and it can be found under various names including "dynamic Monte Carlo" [59, 60], "time-dependent Monte Carlo" [61], and "simple Monte Carlo". The different names are used because they have different origins. They were specifically developed for surface reactions and are based on a dynamic interpretation of equilibrium MC simulations $[60,62,63]$. The popularity and range of applications of KMC has continued to grow and offers an elegant and powerful tool for ex- 
ploring the evolution and properties of a wide range of problems and systems with the aim of faithfully reproducing non-equilibrium, or relaxation processes $[6,64]$. These techniques have been successfully applied to the simulation of the thin film growth [65, 66, 67], irradiation[26, 68], chemical reactions [69], the kinetics of colloidal aggregation [70], phase separation [71, 72], adsorption and desorption processes [71], diffusive transport [73, 74], heterogeneous catalysis [75], and growth and dissolution of materials.

The kinetic Monte Carlo (KMC) method is generally used to simulate the evolution of a physical system through numerical sampling of (Markovian) stochastic processes (briefly outlined in Appendix A). While the traditional Monte Carlo (MC) method is applied to sample in or close to the thermal equilibrium, KMC has a kinetic character, in that it also evolves the system in real physical time making it possible to study nonequilibrium processes [59, 76]. A connection between MC time-step and real physical time has been discussed within the theory of Poisson processes [60]. The appeal of the KMC method is that it can treat large length and long time-scale kinetic responses while incorporating atomistic information, through appropriately determined transition rates. For example, it is widely used to simulate surface diffusion and growth processes [77], in which the energy barriers for the atomic mechanisms are obtained from atomistic calculations.

\subsection{Motivation: the time-scale problem}

Our focus is on simulating the dynamical evolution of systems of atoms. The premiere tool in this class of atomistic simulation methods is Molecular Dynamics (MD), which corresponds to a numerical integration of Newton's equations of motion [78]. The technique makes it possible to carry out computational and theoretical studies of a range of surface phenomena. MD simulations in particular are capable of revealing the essential 
details of microscopic phenomena as they unfold as a function of temperature, pressure, and other global variables but the application is limited in time and length scales [26, 79]. Since most thermally activated atomistic processes occur in the range of picoseconds, they are best captured with time steps in femtoseconds which limit the total simulation time to a few microseconds. These times are many orders of magnitude smaller than processes happening in the laboratory. For example, epitaxial growth and surface morphological changes take place in minutes and hours and are controlled by atomic processes which are infrequent compared to the atomic vibrational times of picoseconds. Also the applications of MD methodology are especially limited for dissolution processes that occur on much longer timescales [80]. This is the time-scale limitation where MD methods are clearly inadequate for simulating such long time scales [81]. The challenge in molecular dynamics simulations is to find reliable ways to capture infrequent processes and extend them to longer time scales with reasonable computational resources.

A possible alternative is to employ the KMC scheme to overcome this limitation for examining surface phenomena. In such phenomena, the rates of various eligible atomic processes are provided as input by exploiting the fact that the long-time dynamics of this kind of system typically consist of diffusive jumps from state to state. Rather than following the trajectory through every vibrational period, these state-to-state transitions are treated directly, as explained in state-to-state dynamics [26, 79]. The result is that KMC can reach vastly longer time scales, typically seconds and often well beyond. Consequently, the KMC can be used to study stateto-state kinetics much more efficiently than molecular dynamics without significant loss in accuracy by using an accurate list of process rates [82]. Although molecular dynamics simulation can be useful for a much shorter time scale, down to nanoseconds or microseconds [83], the properties of the KMC method make it a powerful and realistic approach at present for the simulation of crystal growth at practical size and time scales. 


\subsection{Basic Principles of KMC}

The KMC simulation algorithm is based on existing computer algorithms used for the simulation of the growth on crystal surfaces [32, 33]. These programs carry out elementary jumps on a virtual grid, to represent real atomic jumps on a crystal surface. The basic steps of all KMC algorithms are the following:

1. random selection of a process with the use of transition probabilities for all possible processes,

2. simulation of the selected transition and the corresponding change of the state vector of the system, and

3. upgrade of the time counter and transition probabilities.

From the mathematical standpoint, the kinetic algorithm of the Monte Carlo method realizes a discrete Markovian model of the evolution of a given physical or chemical system with a continuous time determined from an exponential law for the time elapsed between successive transitions in the system. To model dynamic processes, the KMC methodology must solve the Master Equation. The details of this derivation are briefly presented in Appendix C.

\subsubsection{Master Equation}

The KMC technique can be viewed as a method of solving the Master Equation $[24,76,84,85]$ associated with the transition probabilities $W$,

$$
\frac{\partial p_{n}(t)}{\partial t}=-\sum_{n^{\prime}} W_{n^{\prime} n} p_{n}(t)+\sum_{n^{\prime}} W_{n n^{\prime}} p_{n^{\prime}}(t),
$$

which describes a stochastic process in the Markovian approximation [84]. $p_{n}(t)$ is the probability of the surface being in configuration $n$ at time $t$. $W_{n n^{\prime}}$ is a matrix of transition probability per unit time that specifies the rate 
of the process going from configuration $n$ to configuration $n^{\prime}$ or connecting the two states. A configuration is a distribution of particles on a grid, which models the surface and the adsorbed particles. The first term on the right hand side of the Master Equation (3.1) represents all processes where one moves away from the considered state $n$ at the moment in time $t$ and hence its probability is decreased, while the second term contains all reverse processes which hence lead to an increase of the probability of finding $n$. Two very important criteria have to be satisfied when using the Master Equation, namely detailed balance [86] and steady state. The steady state occurs when the time derivative of the Master Equation is zero, that is, $\frac{\partial p_{n}(t)}{\partial t}=0$. This implies that the sum of all the transitions into a particular state $n$ equals the sum of all the transitions out of a particular state $n^{\prime}$. Thus the steady state condition can be written as:

$$
\sum_{n^{\prime}} W_{n^{\prime} n} p_{n}(t)=\sum_{n^{\prime}} W_{n n^{\prime}} p_{n^{\prime}}(t)
$$

The transition probability contains the details of the microscopic processes, and the usual way to calculate $W_{n n^{\prime}}$ is by using the detailed balance principle. The condition of detailed balance is

$$
W_{n n^{\prime}} p_{e q n^{\prime}}=W_{n^{\prime} n} p_{e q n} \Longrightarrow \frac{W_{n n^{\prime}}}{W_{n^{\prime} n}}=\frac{p_{e q n}}{p_{e q} n^{\prime}} .
$$

Equation (3.3) implies that the ratio of the transition probabilities for a move $n^{\prime} \rightarrow n$ and the inverse move $n \rightarrow n^{\prime}$ depends only on the energy change. It is important to impose detailed balances to ensure that the Monte Carlo transition probabilities $p_{e q}$ are consistent with the Boltzmann distribution $[64,87,88,89]$

$$
p_{e q n}=Z^{-1} \exp \left[\frac{-H(n)}{k_{B} T}\right],
$$


where $H$ is the Hamiltonian of the system, $T$ is the temperature, $Z$ is the partition function of the system, and $p_{e q} n$ is the probability distribution of configuration at equilibrium. In Figure 3.1 the difference between the steady state condition property and detailed balance is illustrated. The lengths of the arrows are proportional to the transition rate. Figure 3.1 (a), the anticlockwise transition proceeds at twice the rate of the clockwise transition; therefore the steady state condition holds but the detailed balance is not given. In comparing this to Figure 3.1 (b), both transitions occur at the same rate, thus the detailed balance is satisfied as well as the steady state. Note that detailed balance is a necessary but not sufficient condition for thermodynamic equilibrium.

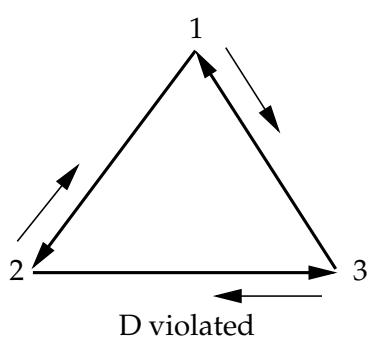

(a).

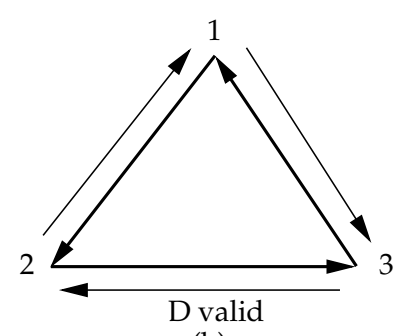

(b).

Figure 3.1: The difference between the steady state condition property and detailed balance. The lengths of the arrows are proportional to the transition rate. In (a), the steady state is satisfied but the detailed balance is not, whereas in (b), they are both satisfied.

\subsubsection{Solution of the Master Equation}

Equations $(3.2,3.3)$ do not uniquely specify transition probabilities. Kang and Weinberg $[88,62]$ have shown different sets of transition probabilities that would eventually lead to the same equilibrium state via different trajectories. The appropriate choice of transition probabilities is important because it is possible to have systems, described by different paths toward equilibrium, even though the equilibrium distribution of configurations is 
the same. The physical trajectory, the one that corresponds to the physical changes in the system, is needed to model dynamic, non equilibrium processes. Kang and Weinberg [87] show that the Master Equation is frequently solved stochastically with algorithms such as Metropolis [90] or Kawasaki dynamics [91, 92,93]. Either of these approaches provides solutions to the Master Equation that can generate the equilibrium configuration, however neither correspond to the physics of the system during the path to equilibrium. Thus, the transition probabilities must be constructed from rates that have physical meaning.

Fichthorn and Weinberg [60] show that the transition probabilities must be formulated to correspond to the physical rates of the microscopic processes to arrive at the physical trajectory. They also show how the theory of Poisson processes can be used to obtain a relationship between the rates of the transitions and the real time step taken in between the MC step. These processes can be grouped together by certain distinctive events,

$$
E=\left\{e_{1}, e_{2}, \ldots, e_{n}\right\}
$$

which can be characterized by average transition rates

$$
R=\left\{r_{1}, r_{2}, \ldots, r_{n}\right\}
$$

From Equations (3.4) and (3.5), it can now be assumed that any particular transition which is possible at time $t$, can potentially occur at any later time $t+\Delta t$ with a uniform probability based on its rate and independent of any previous events. This is, by definition, a Poisson process [85].

\section{One-Step Process}

The Poisson process is part of a family of Markov processes that are called one-step processes. These processes are continuous in time, their range consists of integers $n$, and only jump between adjacent states. Figure 3.2 
helps to visualize a one-step process. The Master Equation for such pro-

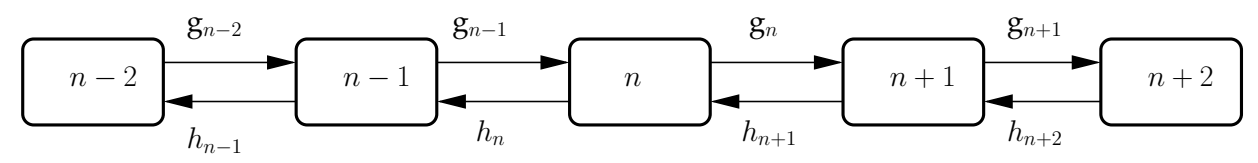

Figure 3.2: Schematic illustration of a one-step process. Only jumps between adjacent states (labelled $n$ ) are allowed. The probability per unit time for jumps in the forward and reverse direction is denoted by $g_{n}$ and $h_{n}$ respectively.

cesses is written as

$$
\dot{p}_{n}=h_{n+1} p_{n+1}+g_{n-1} p_{n-1}-\left(h_{n}+g_{n}\right) p_{n},
$$

where $h_{n}$ is the probability per unit time for a jump from state $n$ to state $n-1$ and $g_{n}$ is the probability per unit time for a jump from $n$ to $n+1$. One step processes occur at:

- generation and recombination processes of a charge carrier;

- single-electron tunneling, and

- surface growth of atoms.

Based on the coefficients $h_{n}$ and $g_{n}$, one-step processes can be subdivided into the following categories:

- linear for coefficients that are linear functions of $n$;

- nonlinear for coefficients that are nonlinear functions of $n$, and

- random walks for coefficients that are constant.

An example of a random walk is the Poisson process which calculates the probability of $n$ events occurring at time $t>0$. This event could be for 
example the tunneling of electrons through a single barrier. The Poisson process is defined by setting

$$
h_{n}=0, \quad g_{n}=q, \quad p_{n}(0)=\delta_{n, 0},
$$

where $q$ is a constant. The Kronecker delta indicates that the probability for no events to occur after time zero equals one, and the probability of more than one event occurring after time zero equals zero. Figure 3.3 shows this Poisson process. For the Poisson process where events are independent,

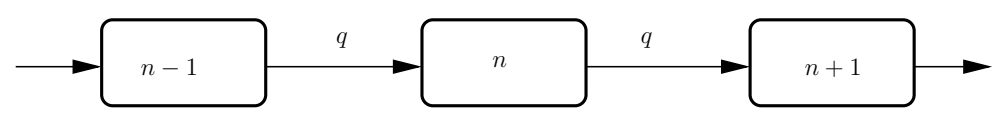

Figure 3.3: Schematic illustration of a Poisson process.

the probability of $n$ events happenening depends only on the time interval. Thus, the probability for one event happening in the time interval $\Delta t$ is $P(n=1, \Delta t)=P(1, \Delta t)=q \Delta t$. Based on these definitions

$$
\begin{aligned}
P(n, t+\Delta t) & =P(n, t ; 0, \Delta t)+P(n-1, t ; 1, \Delta t) \\
& =P(n, t)(1-q \Delta t)+P(n-1, t) q \Delta t \\
P(n, t+\Delta t)-P(n, t) & =q \Delta t\left(p_{n-1}-p_{n}\right) .
\end{aligned}
$$

Thus, the Master Equation for the Poisson process has the form:

$$
\dot{p}_{n}(t)=q\left(p_{n-1}-p_{n}\right)
$$

which has the following solution (the proof of this solution is presented in Appendix D) given by Fichthorn et al. [60]:

$$
p_{n}(t)=\frac{(q t)^{n}}{n !} e^{-q t} .
$$


In the context of transition rates, $q$ can be set to $R$, thus

$$
p_{n}(t)=\frac{(R t)^{n}}{n !} e^{-R t} .
$$

In Equation (3.10), it can be seen that a mathematical adaption of these very basic assumptions leads to the characterisation of a stationary series of random, independent events occurring with an average transition rate $R$ in terms of a Poisson process [94, 95]. It can be shown that a Poisson process is consistent with the Master Equation [84]. Thus, the probability density between successive events is given by

$$
p(t)=R e^{-R t} .
$$

From the probability density, the mean time between successive events is calculated as $\langle t\rangle=1 / R$. However, it may also be of interest to know the real time it takes for an event to occur. This can be deduced by integrating Equation (3.11) with respect to $t^{\prime}$. Since $e^{-R t^{\prime}}>0$ for all $t^{\prime}$, the probability for an event to occur within time $\tau$ is given by

$$
T(\tau)=\int_{0}^{\tau} R e^{-R t^{\prime}} d t^{\prime}=1-e^{-R \tau}
$$

This probability lies in the interval $[0,1]$. The probability for an event not to occur within time $\tau$ is expressed as $T^{*}(\tau)=1-T(\tau)$, which implies $T^{*}(\tau)=$ $e^{-R \tau}$. Thus, $\ln (T(\tau))=-R \tau$. Therefore, it follows that one can relate time to sampling distribution. Since $T^{*}(\tau)$ can be any number (random) in the interval $[0,1]$, the real time, $\tau$, between successive events is calculated as:

$$
\tau=-\frac{\ln U}{R},
$$


with $U=e^{-R \tau}$ a random number uniformly distributed between [0,1]. The overall rate $R$ to determine all processes $i$ of the system is further given by

$$
R=\sum_{i=1}^{n} R_{i}
$$

where $R_{i}=n_{i} r_{i}$ is the macroscopic rate associated with process type $i$ and $r_{i}$ is the microscopic rate associated with process $i$, while $n_{i}$ is the number of particles in the system that are candidates for this process type.

Using the above theory, the KMC simulation can be created that models the evolution of surface morphology of crystal growth. KMC has a direct relation to real time rather than the steps of MC. It can consequently be used to study dynamic processes, in particular those where energy barriers govern the transition between subsequent states. Having laid the conceptual foundation, it is now necessary to discuss the KMC scheme for crystal growth and the implementation of the KMC simulation.

\subsection{KMC Scheme for Crystal Growth}

KMC limits our model to a molecular length scale, but allows us to employ a much bigger time step than could be afforded in MD. In this research, $\mathrm{KMC}$ is used to simulate crystal growth of a face-centered cubic (FCC) crystal. Each discrete lattice site can either be unoccupied, or occupied by a single atom. Starting from a given initial configuration, each time step consists of choosing a particle at random and moving it to a randomly chosen nearest neighbour site. If the chosen site is already occupied, one of the eleven neighboring sites is chosen at random. If this site is occupied, one of its neighbours is chosen at random and so forth until an empty site is found.

The KMC is a powerful method to describe the crystal growth, provided that all relevant processes contributing to growth are considered. It can be very difficult to determine all the relevant processes for some 
situations, particularly if concerted multiple atom rearrangements are involved. Omission of some processes will result in unreliable information about size distribution and morphological behaviour of the crystal growth. The relevant processes that can occur during the crystal growth related to the current study were pointed out in Chapter 2. In addition, an adatom diffusing along a cluster edge or tip can occupy a certain site at a particular time. Specific nomenclature can be assigned to these sites which can be used to distinguish between the different diffusion processes that can occur along a cluster edge or tip, also called edge diffusion (see Figure 3.4). Following this nomenclature, Figure 3.5 illustrates different steps of diffusion processes.

(d)

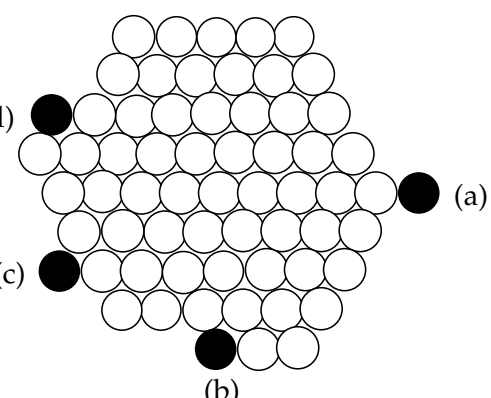

Figure 3.4: Various types of atom structures that are attached to cluster edges and special sites the adatoms can occupy (a) corner site, (b) kink site, (c) step adatom and (d) step dimer.

The movement of each adatom along the surface is determined by its immediate surroundings (nearest neighbour sites). The adatom on the surface of the cluster is allowed to move (by randomly selecting a direction) into any of the nearest neighbour positions not already occupied by an atom. Once the direction is selected, hop is performed. The hopping rate, $p$ (briefly discussed in the next Chapter), is defined according to transition state theory:

$$
p=v_{0} \exp \left[-\Delta E / k_{B} T\right],
$$




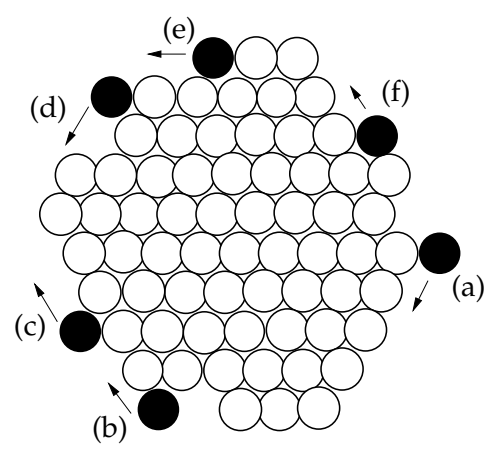

Figure 3.5: Various edge diffusion processes: (a) corner diffusion, (b) corner crossing, (c) step diffusion, (d) kink association, (e) kink dissociation and (f) dimer dissociation.

where $v_{0}$ is the prefactor that is usually modeled as a constant, $k_{B}$ is the Boltzmann's constant, $T$ is the absolute temperature, and $\Delta E$ is an energy barrier that must be overcome in moving from a local energy minimum to an adjacent one in the system's configuration space. The motion of individual atoms takes place instantaneously and the motions are completely independent. With respect to the rare jumps between the basins, the system thus performs nothing but a simple Markov walk [84].

\subsection{Implementation of the KMC Algorithm}

The principal KMC algorithm is based on the method of Bortz, Kalos, and Lebowitz (BKL) [96], with the implementation of an efficient binary search described by Blue, Beichl, and Sullivan (BBS) [97]. A review of KMC studies and algorithms is given by Levi and Kotrla [24]. The basic idea of the BKL algorithm is that, at each MC step, one process is selected with its corresponding probability and then realized, to circumvent the problem of small acceptance probabilities. The BKL algorithm builds on the assumption that the model features $M$ independent Poisson processes with rates $r_{m}$ that sum up to give an overall rate $R$ which can be used to:

1. decide which event to execute, and 
2. randomly select the time it takes for that event to occur from a Poisson distribution.

After generating a random number $r \in[0, R)$, a linear search is used and the required time is $O(M)$, whereas the binary searching time requires $O(\log M)$. Maksym [98] proposed a faster algorithm based on using conditional probabilities to select the sites at which events occur, considering groups of events instead of individual events. He grouped the $M$ rates into $N$ subsets, performing a linear search on the smaller sets. The binary search algorithm is a generalization of Maksym's method which repeatedly subdivides the subsets. To estimate computer time demands, both Levi and Kotrla [24] and Blue et al. [97] give the computation time for Maksym's algorithm as $O\left(M^{1 / 2}\right)$. As previously implemented, this is correct, but the development presented by Schulze [99] shows that Maksym's method can be further adapted, reducing the computation time to a fixed cost per simulated event.

The basic algorithm must be combined with a scheme to determine the rates. One of the most common approaches is to use bond counting although there are other schemes that can be used. The rates can be specified in a look up table for example, but in this study we are focusing only on bond counting scheme because of its simplicity. The rates in bond counting models depend only on the bond count. Typical schemes are dependent on the definition of nearest neighbour interactions and, as a result, only a relatively small number of distinct rates can be found. This result can be used to improve the basic algorithm by initially sorting the possible events according to their rates and then doing some efficient bookkeeping as the simulation proceeds. In KMC, the linear searching scheme agglomerates all the transitions into one large list that is referenced by the partial sum of the rates from the first transition to the transition at a given spot in the list. This methodology solves the problem of sampling and rejecting transitions, as a transition is picked for every random number chosen. Another efficient searching scheme is to utilize a binary tree to keep track 
of all transitions on the surface of the crystal. The binary tree approach has the advantage of both efficient searching and efficient updating. The final step in the implementation procedure is to combine the principles of the KMC method with the assumptions, identified processes and data structures in an algorithm which is capable of simulating crystal growth. Different approaches can be followed to simulate growth, ranging from conceptually very simple to more complex.

In this study we follow closely the version developed by Schulze [99, $100,101]$. The algorithm is described in a general context. In KMC simulations the rates $r_{m}$ are a function of the surface configuration which consists of a set of integer height values. The nearest-neighbour models can assume only a relatively small number of local configurations that affect a given rate. Let this number, which is a constant determined by the specific bond-counting scheme, be $N$, relabeling the much smaller set $(N<<M)$ of distinct rates $R_{n}$. As the simulation proceeds, we maintain $N$ lists in an array $L_{b k}$ that contains the event indices $m$ of events which occur with rate $R_{n}$. We also maintain an address list $A_{m}$ which directs us where event $m$ is currently listed in the array $L_{n k}$ and count $C_{n}$ of the number of events in each of the $N$ lists. A simple description of the KMC algorithm proceeds as follows:

1. Determine all processes $n$ that could possibly take place based on the current configuration of the system.

2. Calculate the overall rate $R=\sum_{n=1}^{N} R_{n} C_{n}$, with a uniform distribution. Deposition or solidification can be one of these processes.

3. Compute the partial sum $S_{k}=\sum_{n=1}^{k} R_{n} C_{n}$.

4. Select a random number $r_{1} \in[0, R)$.

5. Search and compare $r_{1}$ with the list of partial sums $S_{n}$ until $S_{n-1}<$ $r_{1}<S_{n}$. 
6. Select an event from the set of events that occur at this rate by computing

$$
m=\operatorname{Int}\left(\frac{S_{n}-r_{1}}{R_{n}}\right)+1 .
$$

7. Execute that event and update the system configuration and any data structure being used.

8. Select a new random number $r_{2} \in[0, R)$.

9. Update the time with $t=t+\Delta t$ where $\Delta t=-[1 / R] \log \left(r_{2}\right)$.

10. Return to step 2 until the present target simulation time has been reached.

The search in step 5 is necessary to ensure that the process allows the random choice of an event from the event list for each calculation in the simulation. It is also necessary to determine the time step in the simulation. In KMC simulation, we need to somehow introduce the physical time, so that all physical processes are separated and at any time instance, only one event takes place, and all the events are Poisson processes [84]. The time interval $\tau$ between two successive events is a random variable with the distribution $P(\tau)=R \exp (-R \tau)$, and the average value $\langle\tau\rangle=1 / R$. This consideration allows us to generate a time increment between two events in KMC [6]. During the execution of the processes, we generate another random number $r_{2}$ and calculate a time interval $\Delta t=-[1 / R] \log \left(r_{2}\right)$, and this allows us to produce a number of time steps.

\subsection{Summary}

Kinetic Monte Carlo is a powerful method that can be used to study dynamic systems. The evolution of crystal morphology, the physical transitions on the surface, such as adsorbtion, reaction, diffusion and desorption 
and so forth (mentioned earlier) can be studied using KMC simulations. An exact correspondence between real time and simulation time enables the use of KMC simulations to understand experimental surface science and growth. While the focus of KMC techniques in simulations of surface reactions has mainly been in their generic critical and kinetic behaviour, the method is a potentially powerful tool in detailed studies of complex reaction systems. When combined with experimental studies, the techniques can uncover the key microscopic factors controlling the overall reactivity and product distributions. 


\section{Chapter 4}

\section{Deposition and Growth KMC}

The dynamics of the deposition process are complex, measurements are noisy and slow, and disturbances due to contamination and drift limits the repeatability of the material properties that determine device performance [102]. Simultaneous deposition of different types of atoms is widely encountered in experiments and practical applications [103, 104, 105, 106, 107]. The deposition processes can control the structural evolution of the growth surface and can be modelled using the KMC method. The KMC method can be used to simulate the growth processes involving a large number of atoms over a broad time scale [71, 108, 109] at various temperatures. This makes it ideal for calculating the process of deposition which occurs over a long time $(\mathrm{min})$ on a large surface $\left(\mathrm{mm}^{2}\right)$. KMC has been used to model various deposition processes including metal deposition [110, 111], and thin film deposition [112, 113, 109]. The basic processes included in the current model are deposition of atoms and subsequent surface diffusion.

This chapter thus commences with a discussion of what is to be expected for the growth of the nanoparticles in the gas of atoms when the deposition event is included in the KMC algorithm. We focus on an FCC nanoparticle, incorporating the basic assumption that atoms arrive onto the surface of the nanoparticle in a stochastic manner as the result of the 
deposition process. Experiments have shown that the final cluster structure depends on the growth rate $[114,115]$. The rates are applied in the KMC algorithm under conditions similar to those found in the previous work of Combe et al. [116], where KMC was used to examine the evolution of an FCC nanoparticle toward its equilibrium Wulff shape, and the computationally efficient event selection method of Schulze [99]. It is of interest then to study the nanocrystals growth under conditions that might occur in an inert-gas aggregation (IGA) source.

\subsection{Calculating the Hopping Rate}

Most of the deposition simulations of interest are conducted under conditions where thermally activated diffusion processes are occurring. Therefore, along with the deposition events, surface diffusion by all available surface atoms must be taken into account during a time interval. The classical dynamics of the hopping atom and its environment can be described by a classical transition state theory approach [117, 118] (briefly outlined in Appendix E), wherein the motion of the atom is assumed to consist of independent, randomly oriented hops between adjacent binding sites. In this case, the KMC simulation utilizes a standard bond counting algorithm $[116,119,120]$ and the computationally efficient event selection method of Schulze [99]. The number of initial neighbours, $i$, determines the possible events and rates of these events with the configuration after the hop having no influence on the diffusion. The activation barrier an atom must overcome in order to move is $\Delta E=E_{0} \cdot i$, where $E_{0}$ is the energy of a single bond. We adopt the simple model in Reference [116] with $\Delta E$ proportional to the coordination number. The rate $r_{h o p_{i}}$ of a hop in an 
FCC lattice is given as:

$$
r_{h_{o p}}= \begin{cases}v_{0} \exp \left[-\Delta E / k_{B} T\right], & \text { if } i<12 \\ 0, & \text { otherwise }\end{cases}
$$

where $v_{0}=\frac{k_{B} T}{h} \approx 10^{13} \mathrm{~s}^{-1}$ at $500 \mathrm{~K}$ is a prefactor that corresponds approximately to an atomic vibrational frequency and can be thought of as an attempt rate for barrier crossing and $\mathrm{h}$ is Planck's constant. Atoms with many neighbours have smaller hopping rates than atoms with only one or two neighbours, hence they diffuse more slowly. This follows the principle that atoms in regions of positive curvature (that is, high chemical potential) have fewer bonds and diffuse to regions of negative curvature (lower chemical potential). In the current simulation it is assumed that $E_{0}=0.1 \mathrm{eV}$, which is an average value garnered from calculations of diffusion barriers for the $\mathrm{Al}(111)$ surface and which is used extensively in previous works $[101,116,119,120]$. The motivation for choosing the value of the bond energy $E_{0}$ came from noticing that our one-barrier assumption gives a good order of magnitude of the relative jump frequencies for the different hopping process of interest in this simulation.

The rate of different possible events can be influenced by varying the absolute temperature $T$. For example, from Equation (4.1), raising the value of $T$ reduces the difference in the rates of diffusion of an atom with three neighbours relative to that of an atom with four neighbours. Additionally, increasing the value of $T$ from low to high values activates other particular events. For example, atoms with at least seven neighbours have negligibly small rates at $T=400 \mathrm{~K}$, contributing little to the growth but are relatively free to move at $T=500 \mathrm{~K}$. Simulating crystal growth at different temperatures allows investigations of kinetic effects which affect the relaxation time scale. 


\subsection{Deposition Process and Calculating the De- position Rate}

A kinetic model of deposition requires quantitative information about the deposition and hopping rates. These rates are required in units of reactions per second i.e., $s^{-1}$, for all the viable chemical processes pertinent to the deposition system of interest.

The simulation is based on the situation where we have a nanoparticle sitting in a gas of atoms. The gas of atoms can collide with the surface of the nanoparticle and at the same time, the atoms are able to hop around on the surface of the nanoparticle. Some basic calculations show how often the nanoparticle gets hit by the gas of atoms for given gas pressures and temperatures. The deposition rate is related to the surface area of the particle which means that when the nanoparticle becomes bigger, more atoms are expected to deposit on the surface of the nanoparticle. For the purposes of the simulation, we assume that after an atom lands on the surface of the nanoparticle, the atoms then stick and desorption events are forbidden.

Actually, here we are imagining that we have a supersaturated vapour of atoms condensing into a cluster. They could be inert gas atoms but could also be a vapour of metal atoms. The first step is trying to figure out how often to deposit an atom on the surface of a spherical cluster. To calculate the average time $\tau_{g, c}$ between two collisions of inert gas atoms with the cluster, we use the formula given by Hendy et al. [114],

$$
\tau_{g, c} \sim \frac{1}{P R_{\text {clus }}^{2}} \sqrt{\frac{m_{g} k_{B} T}{8 \pi}} .
$$

Here $R_{\text {clus }}$ is the cluster radius, which is assumed to be roughly spherical, $P$ and $T$ are, respectively, the pressure and the temperature of the inert gas of atomic mass $m_{g}$, and $k_{B}$ is the Boltzmann constant. For a $2 \mathrm{~nm}$ radius lead cluster in a helium with a pressure of 5 mbar and a temperature 
of $500 \mathrm{~K}$, the time between collisions is approximately $1 \mathrm{~ns}$ [114]. This approximate time corresponds to the deposition rate, $r_{d e p} \sim \frac{1}{\tau_{g}} \sim 10^{9} \mathrm{~s}^{-1}$. Equation (4.2) can be re-written as

$$
\tau_{g, c} \sim \frac{1}{N^{2 / 3}} \tau_{0}, \quad \text { where } \tau_{0}=\frac{\sqrt{2 \pi m_{g} k_{B} T}}{4 \pi P\left(\frac{3}{4 \pi \rho}\right)^{\frac{2}{3}}},
$$

where $N$ is the number of atoms in the cluster and $\rho$ is the numerical density of the cluster. Equation (4.3) assumes that the cluster is spherical.

In the current model, we consider the deposition rate is uniform over the surface of the cluster as opposed to varying from place to place. The deposition rate can be handled separately from the diffusion process. At each KMC step, a process is chosen at random from a list of all possible reaction mechanisms, including the inert gas atoms deposition event. If the deposition event is chosen, an inert gas atom is added to an unoccupied lattice site on the surface of the cluster and must satisfy the deposition conditions.

The second step is how we should include the process of deposition in the KMC algorithm described in Section 3.5. In addition, we develop a simple, straightforward way to implement the KMC algorithm as follows:

1. add the deposition event,

2. decide how to pick an atom to deposit,

3. decide how to choose the location for the deposition atom, and

4. make sure to update the new set of events and any data structure being used.

In the KMC algorithm, we include the deposition events with the deposition rate $r_{d e p}$, which is computed from the inverse of Equation (4.2) into the event list. This event list contains atoms from one up to eleven neighbours as well as the diposition event with the overall rate $R$. The deposition and hopping rates play an important role in the process of crystal 
growth determing whether the atoms are hopping or are being deposited. The number of possible deposits or hops is fixed by the number of sites available on the surface of the cluster. Let us assume the selected random number $r_{1} \in[0, R]$ lands in the partial sum $S_{n}$ of the deposition event list. An event is then selected from the deposition events that occur at this rate by computing

$$
m=\operatorname{Int}\left(r_{1} \times s\right)+1,
$$

where $s$ is the number of possible deposition sites on the surface of the cluster. Note, if the selected random number $r_{1}$ lands in the partial sum $S_{n}$ of the hopping event lists, then we select an event from the set of events that occur at this rate by computing

$$
m=\operatorname{Int}\left(\frac{S_{n}-r_{1}}{R_{n}}\right)+1 .
$$

The next step is to figure out how to choose the location for the deposition of atoms. Obviously this requires observation of the surface of the cluster and a record being kept of all the sites that are available. In a condition for deposition, the sites must be empty and the number of neighbours of the site must be greater than or equal to three. This condition is applied to prevent the evaporation and collision of the atoms and to help to maintain the spherical shape of the cluster. We could say that the probability of being deposited on the site chosen is dependent on the number of neighbours, but in this current simulation we assume that all the sites are equally likely. After finding a suitable site for deposition, we execute the event and update the system configuration and any data structure being used because the neighbours of the deposition site have gained one more neighbour.

The algorithm is illustrated in Figure 4.1, the procedures are described above and in Section 3.5. 


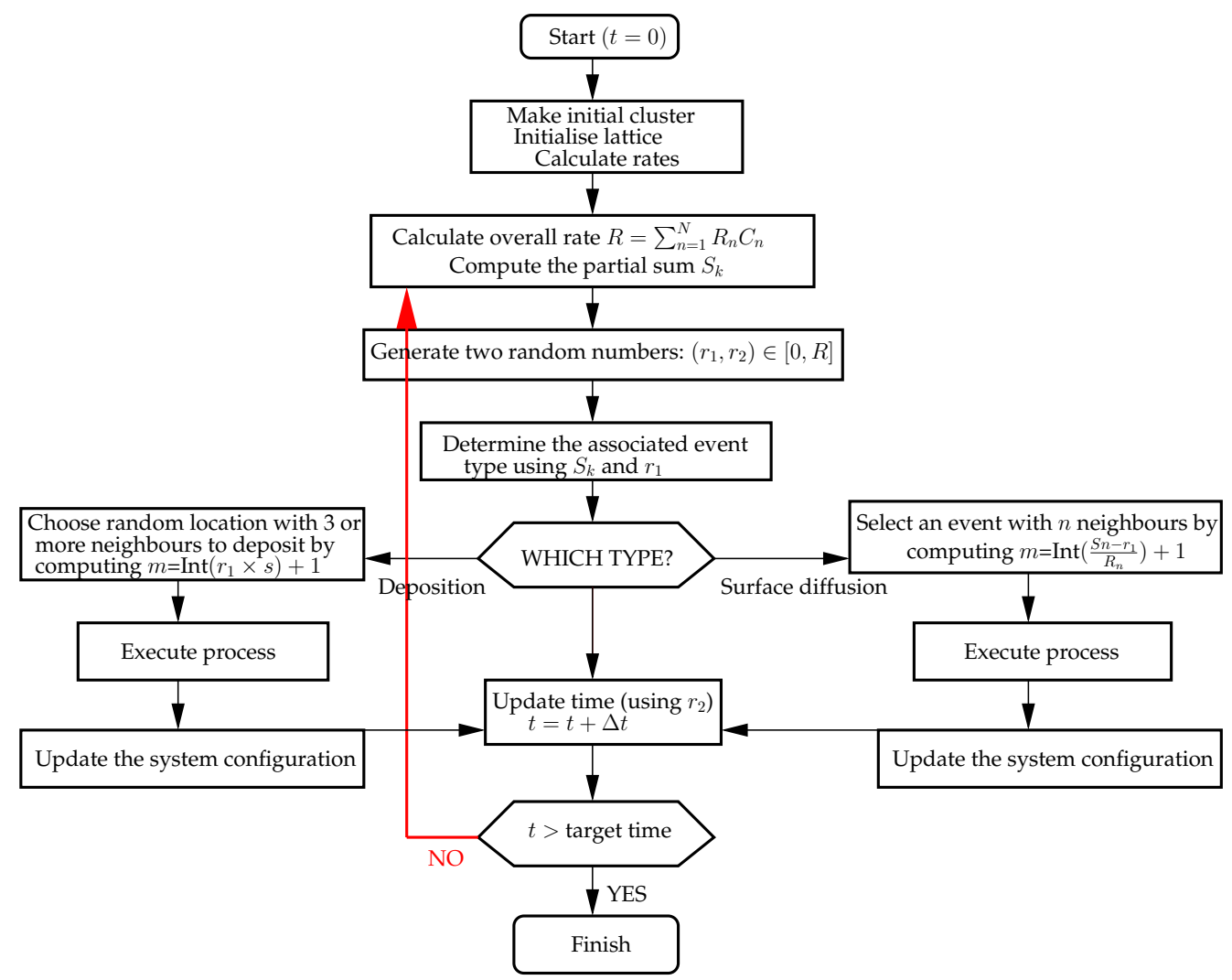

Figure 4.1: The flow chart of the KMC algorithm which includes the deposition event. $r_{1}$ and $r_{2}$ are two random numbers in $[0, \mathrm{R}]$.

\subsection{Results and Discussion}

We have studied the behaviour of nanocrystal structures in a gas of atoms when the deposition event is included. To extend our understanding of the suitability of the KMC algorithm for exploring the evolution of crystal structure, it is necessary to compare the different values of hopping and deposition rates. This comparison will also yield information on how the two rates are controlling the morphology of nanocrystals at a particular temperature. We consider the following three cases:

1. $\mathrm{r}_{\text {hop }}<<\mathrm{r}_{\text {dep }}$, 
2. $\mathrm{r}_{\text {hop }}>>\mathrm{r}_{d e p}$, and

3. $\mathrm{r}_{\text {hop }} \sim \mathrm{r}_{\text {dep }}$.

To ensure that these conditions were satisfied, we varied the vapour density and calculated deposition rate $r_{d e p}$ in each case. The result found from case 1 is understandable by simply observing the values of the deposition rates. For the system described in this chapter, a small spherical cluster of initially about 141 atoms is allowed to grow. The development of the nanoparticle is shown at different instants of time in Figure 4.2. We chose $r_{d e p}=10^{14} \mathrm{~s}^{-1}$ and $T=400 \mathrm{~K}$. Note, that the highest hopping rate of an atom to hop to an unoccupied site is $r_{h o p_{1}}=10^{13} \mathrm{~s}^{-1}$ from Equation (4.1). This unoccupied site has one nearest neighbour. The cluster growth is very fast due to $\mathrm{r}_{d e p}$ dominating the movement of the atoms, and the number of time steps required to reach the final update is therefore shorter.

Figure 4.3 (top) shows the morphologies of the clusters in case 2 when $\mathrm{r}_{\text {hop }}>>\mathrm{r}_{\text {dep }}$. These images correspond to four different times in the simulation that also started from a spherical cluster of 141 atoms. We chose $r_{\text {dep }}=10^{-4} \mathrm{~s}^{-1}$ and $T=400 \mathrm{~K}$. Note, the lowest hopping rate of an atom to hop to an unoccupied site in this temperature is $r_{h o p_{11}}=4.6 \times 10^{-1} \mathrm{~s}^{-1}$ from Equation (4.1). This unoccupied site has eleven nearest neighbours. The hopping of atoms on the surface of the cluster dominates the random movement of atoms, and, as this simulation progresses, the crystallite becomes fully faceted before growth has commenced.

We also examined the different morphologies of the clusters at low $(T=300 \mathrm{~K})$ and high $(T=700 \mathrm{~K})$ temperatures in Figures $4.3(\mathrm{a})$, and (b) and still maintained the condition of case 2 that $r_{h o p}>>r_{d e p}$. We chose the deposition rate, $r_{d e p}=10^{-5} \mathrm{~s}^{-1}$, to be the same for both temperatures. The lowest hopping rate of an atom to hop to an unoccupied site in $T=300 \mathrm{~K}$ is $r_{h o p_{11}}=1.033 \times 10^{-5} \mathrm{~s}^{-1}$ and $r_{h o p_{11}}=5.24 \times 10^{5} \mathrm{~s}^{-1}$ in $T=700 \mathrm{~K}$. This unoccupied site has eleven nearest neighbours. The $\mathrm{r}_{h o p}$ was again dominated by the random movement of atoms on the surface of the cluster. At 
(a).

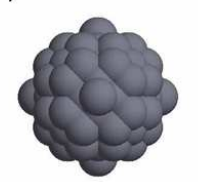

(c).

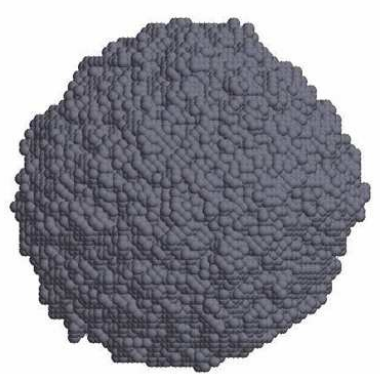

(b).

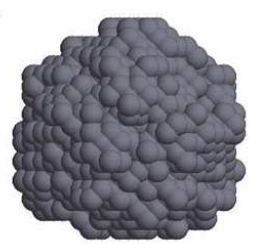

(d).

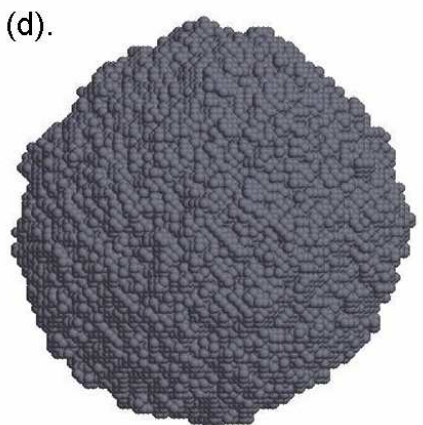

Figure 4.2: Four surface images of a single simulation at four different times and $T=400 \mathrm{~K}$ when $\left(\mathrm{r}_{h o p}\right)<<\left(\mathrm{r}_{d e p}\right)$. The first two images ((a) and (b)) are from the early stages and correspond to the stage at which the cluster contains about 141 and 2412 atoms. The last two images ((c) and (d)) are taken from the late stages, showing the crystal containing about 73500 and $10^{5}$ atoms.

low temperatures (see Figure 4.3 (a)), the crystallite is fully faceted, with angular points and edges, making it difficult to define a chemical potential properly. This would make it difficult to describe using a continuum method. Moreover, the transfer of atoms from the cluster tips or edges to its central regions by simple atomic diffusion is impossible in the presence of facets as the facets do not contain a trapping site. Therefore the atoms eventually move back to kinks or steps at the tips or edges after they reach the facetted regions.

On the other hand, at high temperatures (see Figure 4.3 (c) and (d)) many kinks and steps are present on the surface of the cluster, indicating that the continuous approximation for the curvature might be valid. The number of defects in the core-shell structure is always high. These results indicate the influence of temperature in the process of crystal growth 


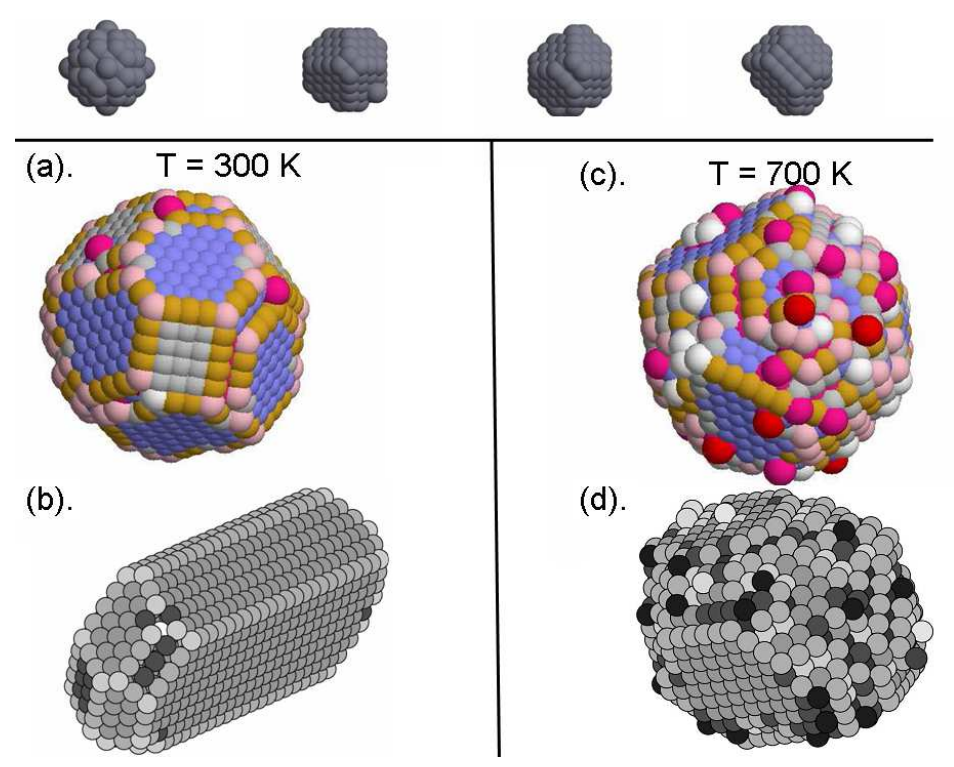

Figure 4.3: (Top). The sequence of the surface images of a single simulation at four different times when $T=400 \mathrm{~K}$. All the clusters contain 141 atoms and the cluster is becoming facetted over time. (Bottom). Morphologies of crystallites of 1728 atoms at two different temperatures: (Bottom left (a) and (b)) fully facetted at $300 \mathrm{~K}$. (Bottom right (c) and (d)) partially rough at $700 \mathrm{~K}$. The atoms that lie on the surface of the clusters are coloured according to their coordination numbers. (b) and (d) were taken from the work of Combe et al. [116].

structure. These simulations are in agreement with the predictions of Combe et al. [116] where their simulations used only the hopping rates. At high temperatures (see Figure 4.3 (d)), many kinks and steps are present on the surface of the particle, and they act as sources of atoms or growth sites for diffusing adatoms. On the contrary, at low temperatures (see Figure $4.3(b))$ the crystallite is fully faceted. 
Figure 4.4 illustrates the sequence of the snapshots of a single simulation at 8 different times that satisfies the condition in case 3 when $r_{h o p} \sim$ $\mathrm{r}_{\text {dep }}$. We chose the value of the deposition rate, $\mathrm{r}_{d e p}=10^{9} \mathrm{~s}^{-1}$, to fall in between the value of the hopping rates to sites with four $\left(r_{h o p_{4}}=1.595 \times\right.$ $\left.10^{9} \mathrm{~s}^{-1}\right)$ and five $\left(r_{h_{0 p}}=8.146 \times 10^{7} \mathrm{~s}^{-1}\right)$ nearest neighbours. This condition can evenly distribute the random selected atoms either to deposit or hop on the surface of the cluster. The growth of the cluster is not as fast as in the first case, but leads to the same structure of spherical shape.
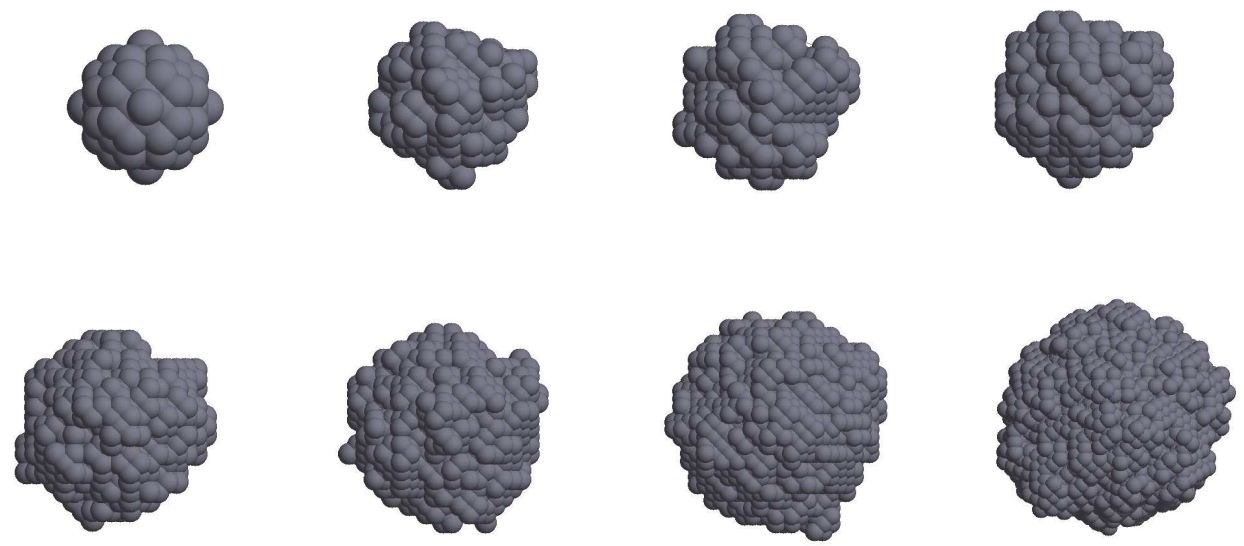

Figure 4.4: The sequence of the surface images of a single simulation at 8 different times when $T=400 \mathrm{~K}$ and $\mathrm{r}_{d e p}=10^{9} \mathrm{~s}^{-1}$. The cluster finally takes on a spherical shape.

We compared the values of three different $r_{\text {dep }}$ in Figure 4.5 to show that the cluster grows much faster when the value of $r_{d e p}$ is increased. The deposition and hopping rates satisfied the condition that $\mathrm{r}_{\text {hop }} \sim \mathrm{r}_{\text {dep }}$. These $r_{\text {dep }}$ were chosen to fall in between the value of the hopping rates to sites which have four and five nearest neighbours at $T=400 \mathrm{~K}$. The solid line indicates the $r_{d e p}=10^{9} \mathrm{~s}^{-1}$, the dashed line shows the result of using $\mathrm{r}_{\text {dep }}=7.5 \times 10^{8} \mathrm{~s}^{-1}$, and the dashdotted line is the result obtained by using $\mathrm{r}_{\text {dep }}=5.0 \times 10^{8} \mathrm{~s}^{-1}$. It can be seen that more atoms were contained in the cluster at any particular time in $\mathbf{r}_{d e p}=10^{9} \mathrm{~s}^{-1}$ compared to the other 

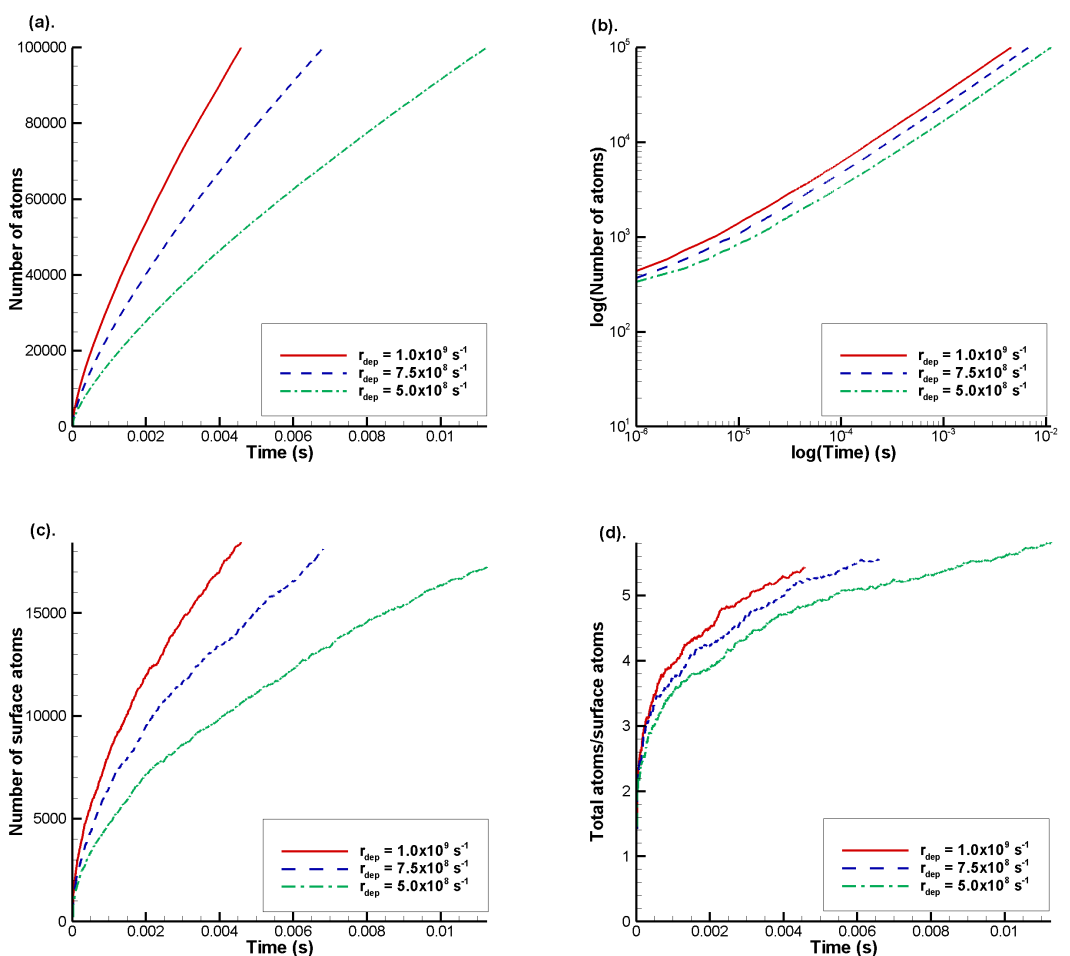

Figure 4.5: This figure shows results for simulations with three different deposition rates. (a) shows the total number of atoms as a function of time. (b) illustrates the log-log plot of the total number of atoms in the cluster against time. (c) shows the number of surface atoms as a function of time. (d) illustrates the ratio of the total number of atoms and surface atoms against time.

two values for $\mathrm{r}_{\text {dep }}$ (see Figure 4.5 (a)). Figure 4.5 (b) shows a log-log plot of the number of atoms in the cluster as a function of time. The power law has a similar exponent of about $2 / 3$ for all three deposition rates after fitting the graph in Figure 4.5 (b). As the $\mathrm{r}_{d e p}$ varies and the temperature remains the same, the slope is approximately the same. Figure 4.5 (c) illustrates that the number of surface atoms is higher when $\mathbf{r}_{d e p}$ increases. To be more clear, we plotted the ratio of the total atoms and surface atoms as a function of time in Figure 4.5 (d). Since at higher deposition rates the cluster is rougher and total number of atoms is higher. Based on the above 
simulation results, we deduce that at low deposition rates the deposited atoms would have enough time to rearrange on the surface of the cluster and form a well-ordered cluster.

\subsubsection{Multiple seeds}

We intend to focus on the merging of two and three identical clusters, a mechanism that can be important for cluster formation, especially in the late stages of the growth process, when already formed clusters can collide and join together. Most approaches have been used previously to study the coalescence of the nanoclusters in favour of enhancing the surface diffusion process [105, 121]. Hendy et al. [114] considered the coalescence of two solid surfaces reconstructed icosahedral clusters. They pointed out that a coalescence process of two solid particles is accelerated, if the temperature of the newly formed particle rises above its melting temperature. More recently, surface diffusion on faceted nanoparticles has been investigated using KMC methods [122, 123]. McCarthy et al. [123] focused their work on the growth of the neck region connecting the two nanoparticles. Their work shows that in the late coalescence stages, when the nucleation of new atomic layers on nanoparticle facets is required for further coalescence, the nanoparticle size, temperature, and nanoparticle orientation all influence the development of the neck. Lim et al. [122] observed the rate growth of the neck that joins two particles during coalescence and makes the comparison with both continuum theory and atomistic KMC simulations. This study can be viewed as the first academic research of coalescence during growth.

We simulate pairs of nanoparticles in a constant temperature environment and address the problems which involves deposition of atoms on the surface of the nanoparticles. We chose $\mathrm{r}_{h o p} \sim \mathrm{r}_{\text {dep }}$ due to its ability to evenly distribute the atom to deposit or hop on the surface of the cluster. The simulations are performed at $T=400 \mathrm{~K}$ and $r_{d e p}=10^{9} \mathrm{~s}^{-1}$, by initially 
placing small spherical particles of 582 atoms and allowing them to grow. The development of the change of shapes is shown at different instants of time in Figure 4.6. Figures 4.6 (a) and (b) are the images after the merge begins, showing the two particles coming into contact and many new bonds forming. As the merge proceeds, the inert atoms and the nanoparticles slowly align with the interface until the icosahedral structure completely disappears in Figure 4.6 (c). Figure 4.6 (d) shows the newly formed structure is now completely spherical.

(a).

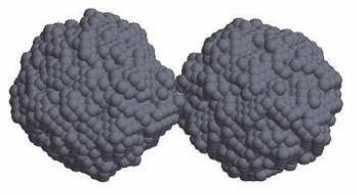

(c).

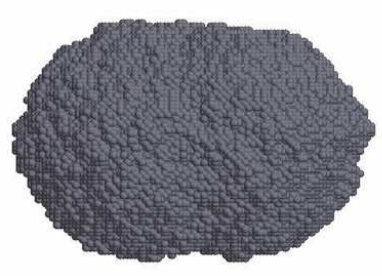

(b).

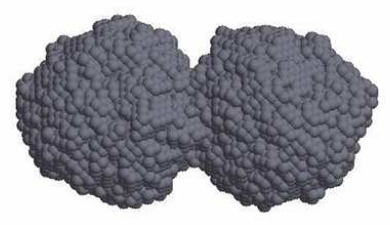

(d).

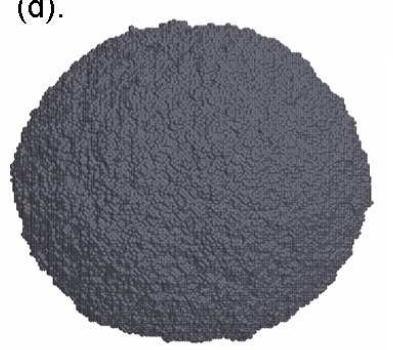

Figure 4.6: Four surface images of a single simulation at four different times when $r_{\text {dep }}=10^{9} \mathrm{~s}^{-1}$ and $\mathrm{T}=400 \mathrm{~K}$. The first two images (a) and (b) are from the early stages and correspond to the stage at which the nanoparticles contains about 6734 and 12268 atoms. The last two images (c) and (d) are taken from the late stages, showing the nanoparticles containing about $10^{5}$ and $10^{6}$ atoms.

An interesting feature is observed on analysing the neck radius of two coalescing Face Centered Cubic (FCC) nanoparticles in Figure 4.7 with $R=4.6$ (the unit of $R$ is the FCC lattice constant). These images were taken from the simulation of Figure 4.6. The neck is marked by two arrows at the neck's apexes, and the distance between the arrows and the 
horizontal line at the center is the neck radius. Figure 4.7 (a) is an image a very short time after the coalescence begins, showing the nanoparticles have reordered from the initial spherical shapes into rough objects. Figures 4.7 (a), (b), and (c) show the neck region to be highly curved, the neck therefore providing a sink for atoms. In Figure 4.7 (d) there are few sites of high coordination available at the neck. Attachment to the neck is easier for depositing atoms but it is more difficult for atoms diffusing on the surface. As time passes, the neck radius increases, until eventually a plateau in neck growth is reached and the two particles form a rod-like oblong shape (see Figure 4.6 (c)).

(a).

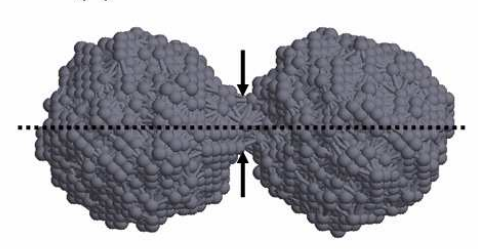

(c).

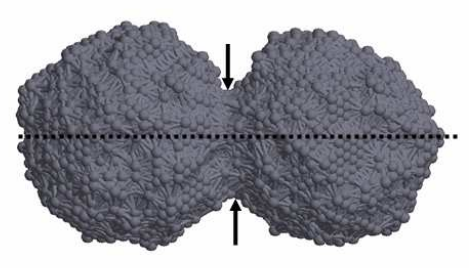

(b).

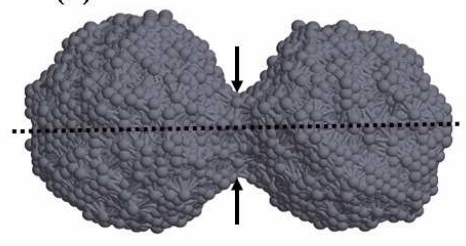

(d).

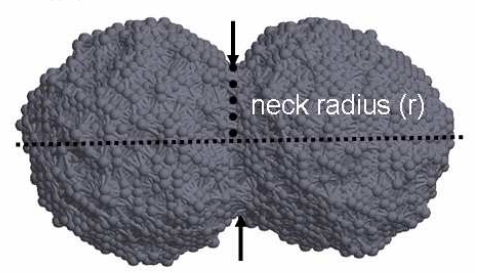

Figure 4.7: Four images taken during the coalescence of two $R=4.6$ nanoparticles when $r_{d e p}=10^{9} \mathrm{~s}^{-1}$ and $\mathrm{T}=400 \mathrm{~K}$. Snapshots are taken at (a) $t=2.05 \times 10^{-4} \mathrm{~s}$, (b) $t=305 \times 10^{-4} \mathrm{~s}$, (c) $t=405 \times 10^{-4} \mathrm{~s}$, and (d) $t=605 \times 10^{-4} \mathrm{~s}$.

Figure 4.8 (a) shows the plot of the neck radius against time and (b) shows a double logarithmic plot of the neck radius as a function of time. The dashed, solid and dotted lines in Figure 4.8 (b) indicate the slope of the power law fit. Assuming a power law relationship $r \alpha t^{a}$, lines with 
slope are drawn for comparison with each approximately linear region of the $\log [r(t)]$ plot. At early stages the neck region is highly curved (see Figures 4.7 (a) and (b)) providing a sink for deposition and surface diffusing atoms with $a \sim \frac{7}{10}$. During the intermediate stage, where $a \sim \frac{2}{5}$, layers are growing around the neck and at the ends of the intermediate phase the volume between the two ends of the dumbell is filled in (see Figure 4.6 (e)). At late stages $a \sim \frac{1}{3}$. The value of $a$ in our model is different compared to McCarthy et al [123]. This is probably because the deposition of atoms is included in the current model, as both models use a similar approach to describing surface diffusion processes. McCarthy et al.'s [123] result have $a \sim \frac{1}{3}$ at early states, $a \sim \frac{1}{6}$ at intermediate states, and $a \sim \frac{1}{9}$ at late states.
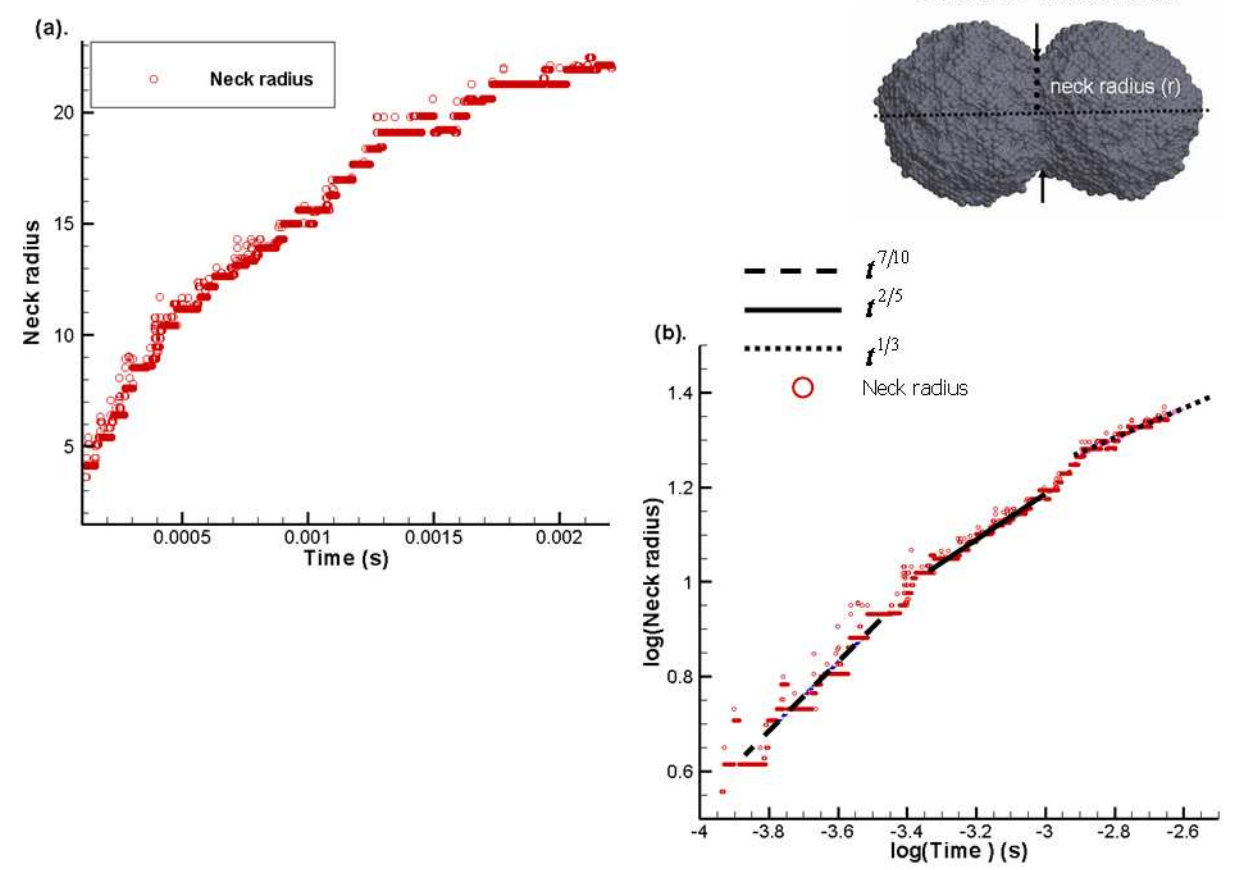

Figure 4.8: (a) Plot of the neck radius against time. (b) double logarithmic plot of the neck radius as a function of time. Power law fits is shown by solid, dashed, and dotted lines in (b)

Figure 4.9 shows the shape changes of three identical seeds when $\mathrm{r}_{h o p} \sim$ 
$\mathrm{r}_{\text {dep }}$. The initial configuration of three seeds is shown in Figure 4.9 (a). The rest of the images (Figure 4.9 (b), (c), (d), and (e)) represent the different configurations of the merging nanocrystals at $T=400 \mathrm{~K}$ from a single simulation. As the crystal becomes larger, the three clusters undergo several different coalescence stages through atomic diffusion and deposition process. Firstly, the three seeds become contacted through diffusion and deposition. Afterward, initial neck regions form among the three clusters and this indicates the beginning of the coalescence (see Figure 4.9 (b) and (c)). Next the inherent icosahedral shapes deform and reorient. Finally, the structure becomes again completely spherical in Figure 4.9 (e).

(a).

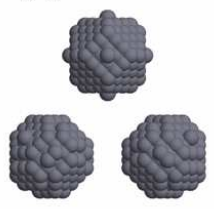

(d).

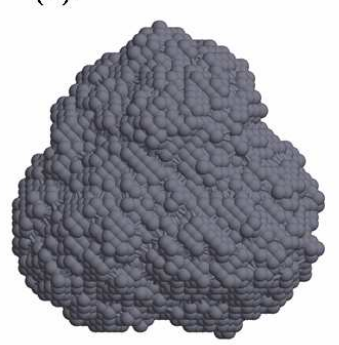

(b)

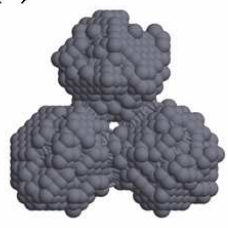

(c).

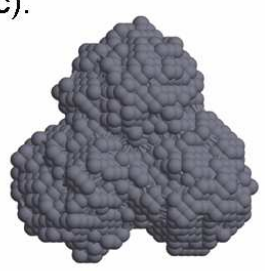

(e).

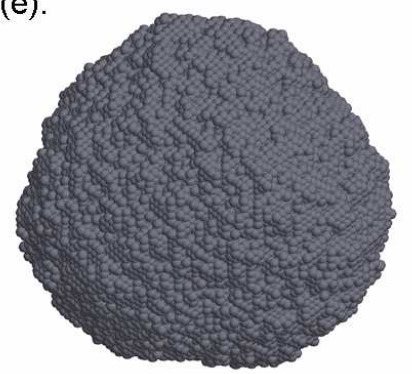

Figure 4.9: Five surface images of a single simulation at five different times when $r_{\text {dep }}=10^{9} \mathrm{~s}^{-1}$ and $\mathrm{T}=400 \mathrm{~K}$. (Top) The top images ((a), (b), and (c)) are from the early stages and the bottom images ((d),(e)) are taken from the late stages. Snapshots are taken at (a) $t=0 \mathrm{~s}$, (b) $t=10^{-5} \mathrm{~s}$, (c) $t=5 \times 10^{-5} \mathrm{~s}$, (d) $t=5.5 \times 10^{-4} \mathrm{~s}$, and (e) $t=4.47 \times 10^{-2} \mathrm{~s}$. 


\subsection{Summary}

In summary, it emerges from our results that atom deposition on the surfaces provides a new step in the understanding of the chemical influence on nanocrystal morphologies. We have identified and discussed the behaviour of nanocrystal growth for different deposition and diffusion surface rates in three cases where $\mathrm{r}_{h o p}<<\mathrm{r}_{d e p}, \mathrm{r}_{h o p}>>\mathrm{r}_{d e p}$, and $\mathrm{r}_{h o p} \sim \mathrm{r}_{d e p}$. The first case, where $\mathrm{r}_{\text {hop }}<<\mathrm{r}_{\text {dep }}$, the cluster growth is very fast due to $r_{d e p}$ dominating the movement of the atoms and the number of time steps required to reach the final time being shorter. The surface image from the late stage is very close to spherical. The second case, where $r_{h o p}>>r_{d e p}$, shows an interesting result in which the crystallite at high temperatures has many visible kinks and steps, indicating that the continuous approximation for the curvature might be valid. This is quite important as it motivates the studies of the next Chapter when the continuous approximation is not valid (that is, at low temperature). We would need to use the type of simulations developed here rather than continuous crystal growth models. On the contrary, at low temperatures, the crystallite is fully faceted, with angular points and edges, making it difficult to define a chemical potential properly. Moreover, the presence of facets makes it impossible to transfer atoms from the cluster tips to its central region by simple atomic diffusion. The final case shows the growth of the cluster is not as fast as in the first case, but leads to the same spherical structure. Snapshots of the nanocrystals station with multiple seeds during early periods (Figure 4.6 and 4.9) show the neck region to be highly curved, providing high coordination sites for material diffusion from the nanocrystal ends. 


\section{Chapter 5}

\section{Continuum-KMC Method}

\subsection{Introduction}

The shape and form of crystal growth into the liquid during the solidification of undercooled pure metals or supersaturated alloys is a topic of interest in physics and materials science. It is typically modeled from a macroscopic point of view as a Stefan problem, where the position of the interface is determined as part of the solution. This is generally used for heat transfer problems with phase-changes such as from liquid to solid. The basic problem involves the free dendritic growth from a circular solid seed placed in a two-dimensional cavity containing an undercooled melt of a pure material. A more complicated problem is to consider free dendritic growth into an undercooled binary alloy [124, 125, 126, 127], where, in addition to heat transfer, the transport of the solute component needs to be considered. During the solidification of a pure substance the solidliquid interface is usually planar, unless severe thermal undercooling is imposed. Such cooling can cause morphological instability, leading to dendritic growth due to the interface growing into an environment that is below the material's melting temperature [128]. This instability leads to the disordered growth of the interface, which causes the branched tree-like solid spikes pattern. Solidification of alloys is more complex than solidi- 
fication of pure substances, as the solidification is limited by both heat and solute diffusion. The heat and solute diffusion fields are coupled at the solid-liquid interface by the relations for the interface temperature (for example, phase diagram) and by heat and solute flux balances.

There are essentially two techniques for approximating the interface:

1. front capturing, and

2. front tracking.

Front-capturing techniques are characterized by treating the interface as a high variation region with no explicit elements to represent the interface. With this approach it is arguably easier to deal with topological changes in the interfaces like merging and breaking. However, a major disadvantage of this technique is the interface diffusion over several cells, resulting in loss of precision. Front tracking methods can deform grids that evolve with the solid-liquid interface [129]. Typically, however, they employ field solutions on a fixed Eulerian background mesh and continuously reconstruct a Lagrangian description of the solid-liquid interface. This interface is tracked in time by explicitly satisfying suitable discrete forms of the interface heat balance condition. The reconstructed interface cuts through the elements of the background mesh and this information is used to modify the background field solution. This can be done by modification of the finite difference [130,131, 132], or finite element approximations [133, 134] in the vicinity of the interface, or through the distribution of interface heat sources [135].

Crystal growth is a classical example of phase transformations from the liquid phase to the solid phase via heat and mass transfers. To understand and simulate crystal growth, several methods have been developed including phase-field [2, 136, 137, 138], level-set methods [3, 139, 140], adaptive mesh techniques [4], random walks with adaptive step sizes [5] and boundary integral [141]. In this study we focus on the front-tracking problem, adopting a discrete, atomistic model in the spirit of the KMC simula- 
tions. This method has been applied recently to simulations of dendritic growth into an undercooled melt [101] of a pure substance which is closely related to the present study. However the current study also addresses the problems which involve undercooling and solute transport. More conventional approaches have been used previously to study the computational modelling of pattern formation in solidification with coupled heat and solute diffusion [131, 133, 142, 143]. Udaykumar and Mao [131] used a mixed Eulerian-Lagrangian frame work that treats the immersed phase boundary as a sharp solid-liquid interface. The spatial discretization is performed using the finite volume method. Special care is required for the treatment of the interface, including the numerical calculation of its velocity and curvature. Zhao et al. [133] performed sharp-interface, two-dimensional simulations of thermosolutal dendritic growth. The heat and solute conservation equations were solved using the finite element method. Echebarria et al. [142] used sharp-interface models for isothermal solidification by considering the solidification of a dilute binary alloy. Yang et al. [143] recently adopted a sharp interface technique to study the interaction of a solid-liquid interface in a solidifying binary alloy with a ceramic particle in the melt. They obtained very realistic growth patterns that captured the development, coarsening and coalescence of primary, secondary and tertiary dendrite arms.

The current study aims to investigate and develop a multiscale simulation method for the growth of nanocrystals in solution. One of the most powerful techniques for studying crystal growth is the KMC method. Over the last decade it has been widely applied to study epitaxial crystal growth with much success $[6,7,8,9]$. However KMC has yet to be widely applied to solution phase crystal growth as, especially with nanocrystal growth, it is necessary to couple the crystal relaxation process with a solute reaction diffusion equation in solution. Here, we consider the combination of growth, surface and solute diffusion for a Face Centered Cubic (FCC) crystal. For convenience, a numerical finite difference method is 
applied, using an explicit discretization to solve heat and diffusion equations. The conservation equations for heat and solute are applied at the solid-liquid interface to solve the interfacial temperature equation. Heat flow is modeled in both phases, on the same FCC lattice, but solute diffusion in the solid is neglected due to the solutal diffusivity being typically two to four orders of magnitude smaller in the solid than in the liquid.

We begin this chapter by describing mass and heat transfer during crystal growth. Next, we describe the mathematical formulation of the heat and diffusion equations together with the boundary equations, then continue to discuss the discrete form of the governing equations. The calculation of solidification and hopping rates are derived, and we present the results and discussion of the simulations which were carried out without consideration of dissolution. Finally, we discuss the dissolution process which is included in the KMC algorithm.

\subsection{Mass and heat transfer during crystal growth}

There are many problems and systems of interest in which heat and mass transfer are accompanied by phase transformation (that is, melting and freezing). Problems of this type are important in crystal growth from melts and solutions. For example, when a crystal is grown by oriented pulling [144] from a melt with alloyed impurities, then the impurity distribution will affect the process of impurity diffusion in the melt as well as in the solid. The contributions of both mass and heat transfer were significant for most of the systems studied, these include aqueous solutions of organic salts and binary organic mixtures. This is supported in most quantitative evaluations which have been carried out in terms of the applications involving crystallization from solution [145, 146, 147, 148]. In particular the heat transfer step was found to be significant for systems having moderate solubilities and heats of crystallization [145]. The effects of mass transfer on the crystal growth from solution have been discussed previ- 
ously in $[147,148]$. The overall solidification process is determined by heat and mass transfer as well as interface kinetics; whether the slow process is nucleation or growth depends on the particular system being considered [149]. The characteristic feature of freezing problems is the coupling of the temperature and concentration fields with the rate of propagation of the phase boundary between the liquid and solid phases.

The situation in studies like the current one is that of a nanocrystal growing in a supersaturated solution or in a supercooled melt. Indeed, before the atoms or molecules pass over from a position in the fluid medium (gas, melt or solution) to their place in the crystalline face they must be transported in the fluid over macroscopic distances. Such mass transport can proceed by diffusion and convection. Further, the heat carried by conductive and convective transport must be dissipated in the solid phase by thermal conduction and radiation to maintain a stable propagating interface [150, 148]. Finally, latent heat of fusion is released when the crystal building blocks dock with the solid phase and lose their fluid phase enthalpy. This heat must also be transported away from the interface. Figure 5.1 gives a more intuitive interpretation of these processes. In theory,

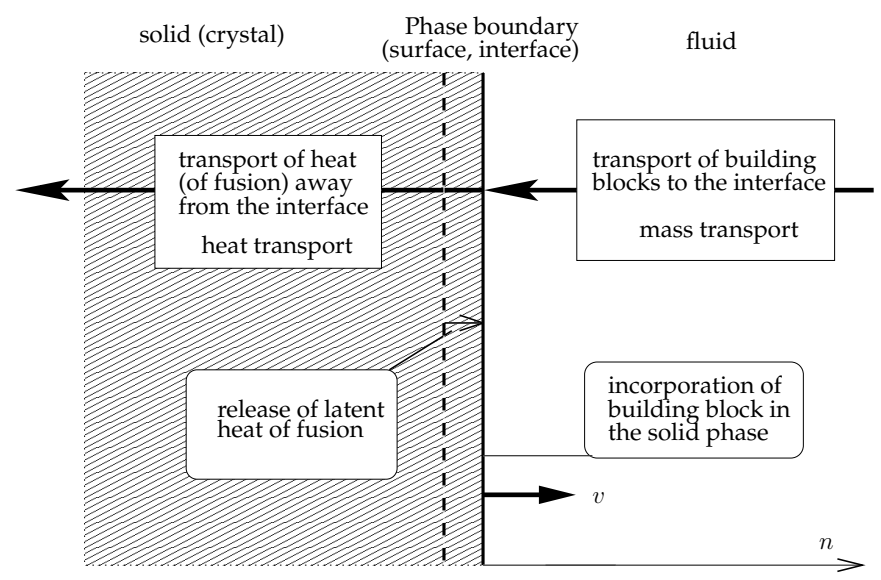

Figure 5.1: Schematic mass and heat transfer situation at the fluid-solid interface with crystallization velocity $v$ along the normal $n$.

the concentration of the major crystallizing component will be lower in 
the liquid near the interface. The differences in the concentration and temperature are the driving forces for mass and heat transfer, respectively. Comprehensive reviews on heat and mass transport in crystal growth are given in [151, 152], for example.

Heat and mass transfer play a vitally important role in all crystal growth processes. The heat transfer problems with phase-changes such as from the liquid to the solid has been mathematically solved by Stefan ("Stefan problem"). In the mathematical formulation of Stefan problems, the curvature effects and the kinetic condition are incorporated with the help of the modified Gibbs-Thomson relation. The former then combines with the mass conservation to calculate the interface temperature which is described in the next section.

\subsection{Mathematical formulation}

The process of crystal solidification, including the effects of undercooling, surface energy, crystalline anisotropy and molecular kinetics, can be described by the mathematical formulation known as the sharp-interface model (see Schulze [101]). In the sharp-interface formulation of the continuum model, the computational domain $\Omega \subset R^{3}$ is typically decomposed into two subdomains. These two subdomains are the interior, solid region $\Omega_{S}$, and the exterior, liquid region $\Omega \backslash \Omega_{S}$, separated by a sharp closed surface $\partial \Omega_{S}$ of zero thickness. The principal governing equations consist of the heat and diffusion equations, with the exterior boundary $\partial \Omega$ representing an infinite reservoir with essentially constant concentration $C_{B}$ which is held at a fixed temperature $T_{B}<T_{M}$. The condition of energy conservation on the moving interface results in a Stefan condition that balances the heat flux away from the interface with the latent heat released. Then the standard set of sharp-interface equation consists of

$$
\partial_{t} T=\alpha_{\mathrm{S}, \mathrm{L}} \nabla^{2} T, \quad \mathbf{x} \in \Omega,
$$




$$
\begin{gathered}
T=T_{B}, \quad \mathbf{x} \in \partial \Omega, \\
\rho L v_{\widehat{\mathbf{n}}}=k\left(\left.\nabla T \cdot \widehat{\mathbf{n}}\right|_{\mathrm{S}}-\left.\nabla T \cdot \widehat{\mathbf{n}}\right|_{\mathrm{L}}\right), \quad \mathbf{x} \in \partial \Omega_{\mathrm{s}} .
\end{gathered}
$$

In the equations above, the subscripts $\mathrm{S}$ and $\mathrm{L}$ denote the solid and liquid phases, respectively. $\left.\nabla T \cdot \widehat{\mathbf{n}}\right|_{S}$ and $\left.\nabla T \cdot \widehat{\mathbf{n}}\right|_{\mathrm{L}}$ are the temperature gradients normal to the solid and liquid sides of interface, $\widehat{\mathbf{n}}$ is the unit vector normal to the interface pointing into the liquid, $v_{\hat{\mathbf{n}}}$ is the value of the normal velocity at which the interface grows locally in the direction of $\overrightarrow{\mathbf{n}}$ and is positive if the solid grows (that is, freezes). $k=\alpha \rho c_{p}$ is the thermal conductivity where the thermal diffusivity $\alpha$ and the density $\rho$ have been assumed to be the same in both phases and $c_{p}$ is the specific heat, $L$ is the latent heat released per unit mass and is independent of the solute concentration.

To describe the diffusion of a single component of a binary alloy, we use the standard dilute-limit diffusion equation. In such situations, the solute diffusion in the solid is several orders of magnitude smaller than in the liquid [142], therefore solute diffusion in the solid is assumed to be negligible. Then the solute concentration is solved for only in the liquid and obeys the standard set of sharp-interface equations:

$$
\begin{gathered}
\partial_{t} C=D_{\mathrm{L}} \nabla^{2} C, \quad \mathbf{x} \in \Omega, \\
C=C_{B}, \quad \mathbf{x} \in \partial \Omega, \\
C_{\mathrm{L}}(1-h) v_{\widehat{\mathbf{n}}}=-\left.D_{\mathrm{L}} \nabla C \cdot \widehat{\mathbf{n}}\right|_{\mathrm{L}}, \quad \mathbf{x} \in \partial \Omega_{\mathrm{s}} .
\end{gathered}
$$

In Equation (5.4) and (5.6) $D_{\mathrm{L}}$ is the solutal diffusion coefficient in the liquid, $h$ is the equilibrium partition ratio, which is assumed to be constant, that is, the solidus and liquidus lines are both assumed to be straight lines, and $\nabla C$ is the concentration gradient evaluated on the liquid side of the interface. During solidification, the melting temperature varies due to changes in solute concentration. With the assumption that phase change takes place under local thermodynamic equilibrium, the temperature at the solid-liquid interface of a binary alloy, that is, the melting temperature 
$T_{M}$, can be expressed

$$
T_{M}=T_{M 0}+m_{\mathrm{L}} C_{\mathrm{L}}
$$

where the liquidus on the phase diagram is assumed to be linear with a slope $m_{\mathrm{L}}, C_{\mathrm{L}}$ is the solute concentration in the liquid phase of the interface and $T_{M 0}$ is the melting point of the major component of the alloy (i.e., the solvent). There is a condition to be satisfied on the solid-liquid interface. This is the generalized Gibbs-Thompson relation. This condition provides the closure needed for the free boundary problem. The interface temperature is related to the interface species concentration via the phase-diagram and is given by:

$$
T_{I}=T_{M 0}+m_{\mathrm{L}} C_{\mathrm{L}}-\frac{\gamma T_{M 0}}{\rho L} \nabla \cdot \widehat{\mathbf{n}}(\mathbf{x}), \quad \mathbf{x} \in \partial \Omega_{\mathrm{s}}
$$

where the subscript $I$ denote the interface, $\frac{\gamma T_{M 0}}{\rho L}$ is the Gibbs-Thomson capillary coefficient where $\gamma$ is the surface energy along the interface line and is usually anisotropic in real material systems and $\nabla \cdot \widehat{\mathbf{n}}(\mathbf{x})$ is the local curvature of the interface. The interfacial temperature is given by the equilibrium melting temperature for a flat interface $T_{M 0}$ modified by a curvature term accounting for surface energy $\gamma$.

We choose dimensionless variables by using $a$ as a reference length scale with $a$ being the distance between neighboring lattice sites, $a^{2} / \alpha$ as the time scale and $\Delta T=T_{M 0}-T_{B}$ as the temperature scale. There are four principal parameters in the absence of surface energy anisotropy. These are surface energy parameter $\widetilde{\Gamma}$, the Stefan number $S t$, the Lewis number $L e$, and the non-dimensional slope of the liquidus $m$ as shown below:

$$
\widetilde{\Gamma}=\frac{\gamma T_{M 0}}{\rho L a \Delta T}, S t=\frac{L}{c_{p} \Delta T}, L e=\alpha_{\mathrm{L}} / D_{\mathrm{L}}, m=m_{\mathrm{L}} C_{0} / \Delta T .
$$

The Lewis number characterizes the relative roles of the thermal and solute diffusion in controlling the solid-liquid interface movement. The nondimensional slope $(m)$ has a part in controlling the interface tempera- 
ture. Note that the velocity field $v_{\widehat{\mathbf{n}}}$ is measured in units of $\alpha / a$ where $\left(\widetilde{v}_{\widehat{\mathbf{n}}} \rightarrow v_{\widehat{\mathbf{n}}} a / \alpha\right)$.

\subsection{The discrete form of the governing equations}

The heat equation in Equation (5.1) is non-dimensionalized as

$$
\frac{\partial T}{\partial \hat{t}}=\frac{\partial^{2} T}{\partial \hat{x}^{2}},
$$

and solved numerically using an explicit Euler time discretization. Then the updated temperature field discretized on the FCC lattice becomes

$$
T_{i j k}^{n+1}=T_{i j k}^{n}+\frac{\Delta t}{2}\left(\sum_{m=1}^{12} T^{n}\left(\mathbf{x}_{i j k}+\mathbf{e}_{m}\right)-12 T_{i j k}^{n}\right) .
$$

Note in Section 2.2, we discussed that the Face Centered Cubic (FCC) lattice can be defined using integer combinations $\mathbf{x}_{i j k}=i \mathbf{a}_{\mathbf{1}}+j \mathbf{a}_{\mathbf{2}}+k \mathbf{a}_{\mathbf{3}}$ of three basis vectors $\mathbf{a}_{1}, \mathbf{a}_{2}$, and $\mathbf{a}_{3}$. In practice one needs only store and manipulate integer triples $(i, j, k)$, converting to Cartesian coordinates for visualization. $\mathbf{e}_{m}$ denote the twelve vectors that point to the nearest neighbours of a given lattice site. We solve the diffusion equation in the same form as the heat equation by non-dimensionalizing Equation (5.4) as follows:

$$
\frac{\partial C}{\partial \hat{t}}=\frac{1}{L e} \frac{\partial^{2} C}{\partial \hat{x}^{2}}
$$

The update of the concentration field discretized on the FCC lattice then becomes

$$
C_{i j k}^{n+1}=C_{i j k}^{n}+\frac{\Delta t}{2 L e}\left(\sum_{m=1}^{12} C^{n}\left(\mathbf{x}_{i j k}+\mathbf{e}_{m}\right)-12 C_{i j k}^{n}\right) .
$$

The solidification model is analogous to the combination of the GibbsThomson equation, Stefan condition and solute conservation given in Equa- 
tion (5.7) and the equation is re-written as

$$
T_{I}\left(\mathbf{x}_{i j k}\right)=1+m C_{\mathrm{L}}+\widetilde{\Gamma}\left(N_{i j k}-3\right),
$$

where $N_{i j k}$ is the number of solid nearest neighbours and $N_{i j k}-3$ is used to measure the curvature of the flat surface on the FCC crystals. Note that an adatom on an FCC surface has 3 neighbours so this definition effectively assigns a "zero" curvature to the (111) facet. In the discrete model, the surface energy can be included by making the melting temperature depend on the number of solid phase nearest neighbours. The melting temperature of pure solvent has been scaled and translated so that $T_{M 0}=1$ and $\widetilde{\Gamma}$ is the surface energy parameter given in Equation (5.8). This is then multiplied by an unspecified geometric factor that translates coordination numbers into a measure of curvature. Note that $T_{M}$ is taken to represent the melting temperature of the (111) facet, as most atoms will solidify or melt along such a facet when they have three nearest neighbours. This temperature is susceptible to phase change when it is above the liquid site and below the solid site. The problem of the solidification into an undercooled binary melt specified above is in this situation applied only in the freezing condition.

It is necessary to note that Equations (5.9) and (5.10) come with the severe time-step restriction of $\Delta t \leq 1 / 6$ for numerical stability. This timestep restriction was selected to facilitate solving Equations (5.9) and (5.10). Thus, we set the maximum value of the time step $\Delta t=1 / 6$. In practice this is not problematic since the KMC typically runs on shorter time scale. We apply an explicit finite-difference method to solve for $C_{\mathrm{L}}$ by re-writing Equation (5.6) in the form

$$
C_{\mathrm{L}}(1-h) v_{\widehat{\mathbf{n}}}=-D_{\mathrm{L}} \frac{\left.\partial C\right|_{\mathrm{L}}}{\partial \widehat{\mathbf{r}}} \cdot \widehat{\mathbf{n}}=D_{\mathrm{L}} \frac{\left.\partial C\right|_{\mathrm{L}}}{\partial \widehat{\mathbf{n}}}
$$


where

$$
\frac{\left.\left(C^{n}\left(\mathbf{x}_{i j k}+\mathbf{e}_{m}\right)-C^{n}\left(\mathbf{x}_{i j k}\right)\right)\right|_{\mathrm{L}}}{\Delta x}=\frac{\left.\partial C\right|_{\mathrm{L}}}{\partial \widehat{\mathbf{n}}} .
$$

The non-dimensionalizing Equation (5.12) reduces to a simple average over the twelve nearest neighbours and we set $\Delta \hat{x}=1$, then

$$
L e(1-h) C_{\mathrm{L}}\left(\mathbf{x}_{i j k}\right) \widetilde{v}_{\widehat{\mathbf{n}}}=\frac{1}{12} \sum_{m=1}^{12}\left[C^{n}\left(\mathbf{x}_{i j k}+\mathbf{e}_{m}\right)-C_{\mathrm{L}}\left(\mathbf{x}_{i j k}\right)\right] .
$$

We re-arrange the Equation (5.13) and solve for $C_{\mathrm{L}}\left(\mathrm{x}_{i j k}\right)$, then

$$
C_{\mathrm{L}}\left(\mathbf{x}_{i j k}\right)=\left(1+L e(1-h) \widetilde{v}_{\hat{\mathbf{n}}}\right)^{-1} \times \frac{1}{12} \sum_{m=1}^{12} C^{n}\left(\mathbf{x}_{i j k}+\mathbf{e}_{m}\right),
$$

where

$$
\widetilde{v}_{\widehat{\mathbf{n}}}=S t^{-1} T_{I}\left(\mathbf{x}_{i j k}\right)-S t^{-1} \frac{1}{12} \sum_{m=1}^{12} T^{n}\left(\mathbf{x}_{i j k}+\mathbf{e}_{m}\right) .
$$

Equation (5.11) may then be rewritten incorporating Equation (5.14), which gives the solution in the form of a quadratic equation

$$
\begin{gathered}
b_{1} T_{I}^{2}\left(\mathbf{x}_{i j k}\right)+\left(1-b_{1}\left(1+b_{2}+\widetilde{\Gamma}\left(N_{i j k}-3\right)\right)\right) T_{I}\left(\mathbf{x}_{i j k}\right)- \\
\left(1+m b_{3}-b_{1} b_{2}\left(1+\widetilde{\Gamma}\left(N_{i j k}-3\right)\right)+\widetilde{\Gamma}\left(N_{i j k}-3\right)\right)=0,
\end{gathered}
$$

where

$$
\begin{gathered}
b_{1}=L e(1-h) S t^{-1}, \\
b_{2}=\frac{1}{12} \sum_{m=1}^{12} T^{n}\left(\mathbf{x}_{i j k}+\mathbf{e}_{m}\right), \\
b_{3}=\frac{1}{12} \sum_{m=1}^{12} C^{n}\left(\mathbf{x}_{i j k}+\mathbf{e}_{m}\right) .
\end{gathered}
$$


By solving this quadratic equation, the melting temperature associated with each lattice site may be obtained. This is

$$
T_{I}\left(\mathbf{x}_{i j k}\right)=\frac{1}{2 b_{1}}\left(B_{1}+\sqrt{B_{2}-4 B_{3}}\right)
$$

where

$$
\begin{gathered}
B_{1}=-1+b_{1}\left(1-b_{2}+\widetilde{\Gamma}\left(N_{i j k}-3\right)\right), \\
B_{2}=\left(1-b_{1}\left(1+b_{2}+\widetilde{\Gamma}\left(N_{i j k}-3\right)\right)\right)^{2}, \\
B_{3}=-b_{1}\left(1+m b_{3}+\widetilde{\Gamma}\left(N_{i j k}-3\right)-b_{1} b_{2}\left(1+\widetilde{\Gamma}\left(N_{i j k}-3\right)\right)\right) .
\end{gathered}
$$

Note, we chose the positive root in Equation (5.15) so that the value of the normal velocity is positive. The combination of the Gibbs Thompson equation, Stefan condition and mass conservation allows us to calculate the melting temperature given in Equation (5.15).

\subsection{Calculating solidification and hopping rates}

The current simulation applies the solidification rate and hopping rate to examine the behaviour of the crystal structure using the KMC algorithm under conditions similar to those found in the previous work of Schulze [101]. Experiments have shown that the final cluster structure depends on the growth rate $[114,115]$. Before a solid can melt it must acquire a certain amount of energy to overcome the binding forces that maintain its solid structure. This energy is referred to as the latent heat of the material and represents the difference in thermal energy levels between the liquid and solid states. Of course, solidification of a liquid requires the removal of this latent heat and the structuring of atoms into more stable lattice positions. In this simulation, liquid sites with temperature $T_{i j k}^{n+1}<T_{I}$ have their temperature pinned at $T_{I}$. Thus, these sites serve as sources of latent heat. In the continuum model, the Stefan condition (5.3) governs 
the amount of heat needed to convert the phase of such atoms. This states that the latent heat released due to the interface displacement equals the net amount of heat delivered to (from) the interface per unit area per unit time. This condition is enforced in the simulations presented below by setting the solidification rate equal to

$$
S_{i j k}= \begin{cases}J\left(\mathbf{x}_{i j k}\right), & \text { if } \sigma_{i j k}=0 \text { and } N_{i j k}>0 \\ 0, & \text { otherwise }\end{cases}
$$

where

$$
J\left(\mathbf{x}_{i j k}\right)=S t^{-1} \frac{1}{12} \sum_{m=1}^{12}\left[T_{I}\left(\mathbf{x}_{i j k}\right)-T^{n}\left(\mathbf{x}_{i j k}+\mathbf{e}_{m}\right)\right],
$$

represents the net heat loss at site $\mathbf{x}_{i j k}$ measured in units of latent heat released per atom upon solidification. We also need to determine how to choose the location for the solidified atoms. This requires observation of the surface of the solid and a record being kept of all the sites that are available. In the condition for solidification, a site must be empty and must have at least one solid neighbour. In that case, an order parameter $\sigma_{i j k} \in\{0,1\}$ is introduced, which is an indicator phase distinguishing the liquid (0) and the solid (1) phase. In addition to this phase configuration, we associate a temperature $T_{i j k}$ and a concentration $C_{i j k}$ with each lattice site to specify the system state.

Anisotropy arises from the model through surface diffusion where solid atoms are able to hop around on the surface when they are in contact with at least one liquid site. These solid atoms are then able to occupy the liquid sites which have one or more solid neighbors. This is similar to the solid-on-solid (SOS) rule in the epitaxy literature which prevents the detachment of the atoms [101]. The hopping atom and its environment can be described by a classical transition state theory approach [118], wherein the motion of the atom is assumed to consist of independent, randomly oriented hops between adjacent binding sites. In this case, each hop to a 
neighbouring site will have a different probability determined by its activation energy barrier $\Delta E$, which in turn is assumed to depend on the change in the local coordination number $N_{i j k}$. Therefore, the rate of a hop is given by

$$
H_{i j k}= \begin{cases}v_{0} \exp \left[-\Delta E / k_{B} T\right], & \text { if } \sigma_{i j k}=1 \text { and } N_{i j k}<12, \\ 0, & \text { otherwise }\end{cases}
$$

where $v_{0} \approx 10^{13} \mathrm{~Hz}$ is a prefactor, $k_{B}$ is the Boltzmann constant and $T$ is the absolute temperature. We adopt the simple model of Combe et al. [116] as mentioned in Section 4.1 that the activation energy barrier $\Delta E=E_{N} \cdot N_{i j k}$ can be calculated by assuming it is proportional to the local coordination number. This is also a common assumption with SOS models for simple cubic growth because it is extremely fast and is easily reproduced by others.

\subsection{Results and Discussion}

\subsubsection{Time step and physical parameters}

It is also necessary to determine the time step in the simulation. In KMC all physical processes are separated so that in any time instance only one event takes place, and the events are Poisson processes [84]. During the execution processes, we calculate a time interval $\delta t=-[1 / R] \log (r)$, and this allows us to quantify the time step. Note, that $r$ is a random number $(r \in[0, R))$ and $R$ is the overall rate that determines all processes of the system. At each KMC time step $\delta t_{n}$, the solidification and hopping rates play an important role in the process of crystal growth. A hopping (exchange) or solidification (flip) event is selected with probability proportional to its rate and a random waiting time is associated with the event. The number of possible exchanges or flips is fixed by the number of sites 
available on the surface of the cluster. When the accumulated time step $\delta t_{n}$ is greater or equal to $\Delta t$, the temperature and concentration fields are updated, using Equations (5.9) and (5.10).

The growth of crystals, coupled with the transport of heat and solute, were computed for a range of physical parameters. The typical values used in the calculations are given in Table 5.1. The inverse of the Stefan

Table 5.1: The parameters used in the present work.

\begin{tabular}{lll}
\hline \hline Symbol & Definition & Value and units \\
\hline$D_{L}$ & Diffusion coefficient & $1.35 \times 10^{-9} \mathrm{~m}^{2} \mathrm{~s}^{-1} \quad[153]$ \\
$\alpha_{L}$ & Thermal diffusivity & $1.0 \times 10^{-6} \mathrm{~m}^{2} \mathrm{~s}^{-1}[153]$ \\
$m_{L} C_{0}$ & Shift in melting temperature & $2.0 \mathrm{~K}[153]$ \\
$k$ & Partition coefficient & $0.3[153]$ \\
$\widetilde{\Gamma}$ & Surface energy & $0.01[101]$ \\
$K$ & Surface diffusion prefactor & $10^{6}[101]$ \\
\hline
\end{tabular}

number $S t^{-1}$ was varied in order to study the merits of using the KMC technique to track the free boundary and $C_{0}$ is the initial concentration. The computational domain is a sphere with a radius of 25 times the radius of the initial solid region, a spherical cluster of about 400 atoms. The initial temperature is set to $T_{M 0}=1$ in the solid and $T_{B}=0$ in the liquid while the initial concentration has been scaled and set to $C_{s}=1$ in the solid and $0 \leq C_{B}<1$ in the liquid. In order to produce realistic dendrite shapes (see Figure 5.2) in this simulation. We found that we must also boost the hopping rates, controlled by the nondimensional parameter $K=v_{0} a^{2} / \alpha$, by a similar order of magnitude. We have briefly investigated the behaviour of the growth as the remaining parameter, the surface diffusion prefactor $K$, is varied (see Figure 5.3). When $K$ is too small then the crystal morphology is completely spherical. The major observation is that the size of $K$ relative to the growth rate controls the extent to which faceting and anisotropy dominate the morphology.

The most relevant parameter in the solution (5.14)-(5.15) is the Lewis 


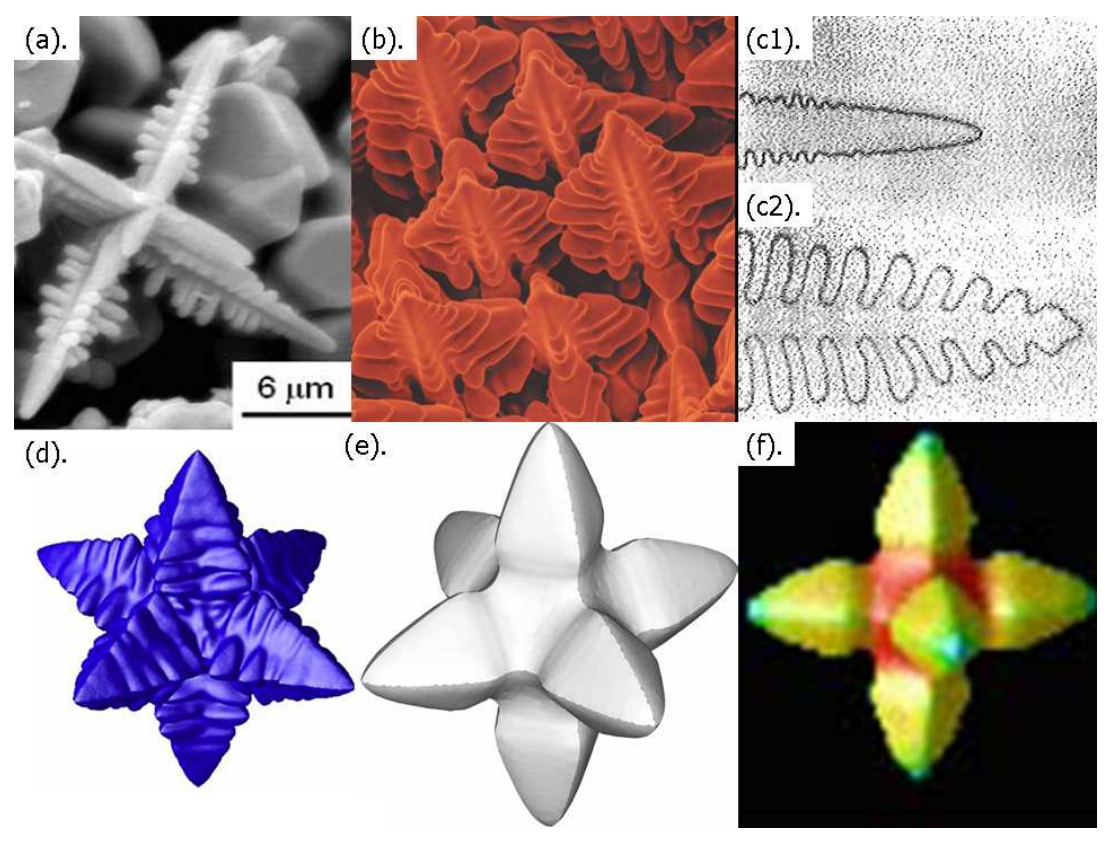

Figure 5.2: Image (a) shows 3D treelike dendrite of TiN microcrystals prepared by combustion synthesis [36]. Image (b), a scanning-electron micrograph showing the development of dendrites in a nickel-based super alloy single-crystal weld [154]. Image (c) shows photograph of 2D growth types; (c1) tip-oscillating type, whose tip curvature oscillates in time; (c2) tipstable type whose tip is a parabola and steady in time [155]. Image (d), 3D $\mathrm{Ni}-\mathrm{Cu}$ dendrite simulation using a thermodynamically consistent phasefield method [156]. Image (e), 3D dendrite crystal shape using a level set simulation [139]. Image (f), 3D dendrite crystal shape using phase-field models [157].

number Le (ratio of thermal diffusivity to solutal diffusivity). This dimensionless parameter characterizes the relative roles of the thermal and solute diffusion in controlling the solid-liquid interface movement. Voller [158] investigated the influence of the Lewis number Le on solidification behaviour and identified the physical limit conditions for the problem. He presented a similarity solution for the solidification of an under-cooled binary alloy melt contained in a semi-infinite insulated slot. Ramirez et al [159] investigated the effect of $L e$ varied from $L e=1$ to $L e=200$. 
(a).

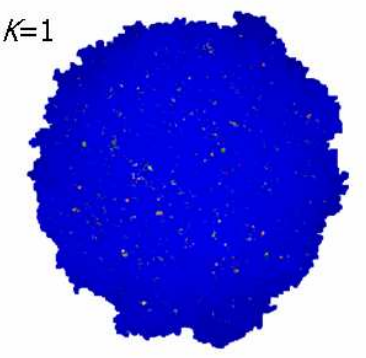

(c). $K=100$

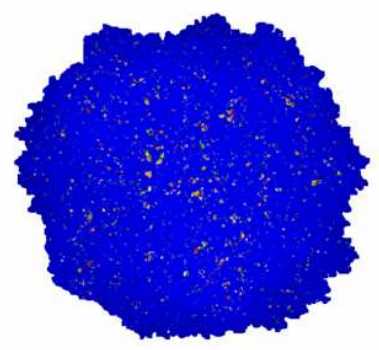

(b). $k=10$

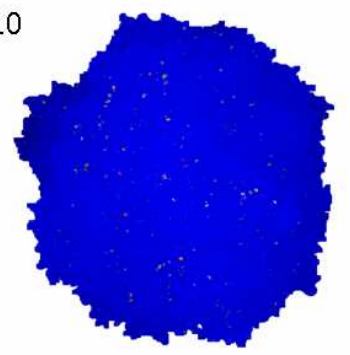

(d). $K=1000$

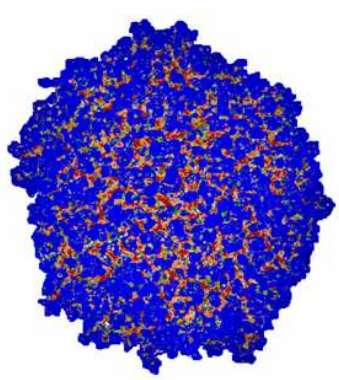

Figure 5.3: Four surface images from the late stages of four separate simulations when $K=1,10,100,1000$ and Lewis number $L e=1$, showing about $10^{6}$ atoms coloured by temperature gradient.

Their work concerned the more general case where heat and solute diffusion simultaneously limit the growth. Such thermosolutal growth is important in solidification of relatively dilute binary alloys, even though the thermal diffusivity may be much larger than the solutal diffusivity. Ramirez et al's [159] simulations were carried out in two dimensions using a structured adaptive grid and nonlinear preconditioning of the phasefield equation.

In the current model, we improve on Voller's [158] one-dimensional and Ramirez et al's [159] two-dimensional models by providing a better three-dimensional model of the interface. We also have an atomistic model of surface relaxation that enhances the effect of the Lewis number. Our model also provides quantitative insights into the effect of the Lewis number and the relative roles of thermal and solute diffusion transports 
during solification.

\subsubsection{Effect of Lewis number}

For the system described above, a small spherical cluster of 531 atoms initially is allowed to grow and the development of the unstable front is shown at different instants of time when Lewis number $L e=1$ in Figure 5.4 (a). The tip of the crystal assumes a parabolic shape that subsequently becomes unstable and generates side-branches, which in turn grow in the preferred direction. The effect of $L e$ is further shown by the series of predicted shapes in Figure 5.4 (b). In this figure the simulation time $t=2400$, is identical for each dendrite. The size and shape is controlled by the Lewis number as expected, where the crystal grows faster and the branching process occurs at earlier stages of the crystal growth when the Lewis number approaches zero. It can be seen from Figure 5.4 (b) that when $L e=1$ the arms of the crystal are bigger than for the other two values of $L e$. This result illustrates that when the value of $L e$ decreases, the number of solidifying atoms are increased. To be more clear, we compared the surface images of three separate simulations taken at the same time when $t=540$ in Figure 5.5. It can be seen that the dendrite arms are already appearing when $L e=1$ and the number of solid atoms are also higher than the other two Le. Note that only the values of $L e$ and $S t^{-1}$ are varied and the rest of the parameters remain the same in the simulations reported in this section (see Table 5.1).

It was found that when the Lewis number Le was decreased it had an effect on the number of solidified atoms, surface atoms and solid atoms in the crystal. Hence the size and shape is controlled by the Le. This was established by plotting the number of solid atoms against time and by observing the relationship for different $L e$ values. These graphs can be found in Fig. 5.6 (a). It can be seen from the graphs in Fig. 5.6 (a), that more solid atoms were contained in the crystal at any particular time in 

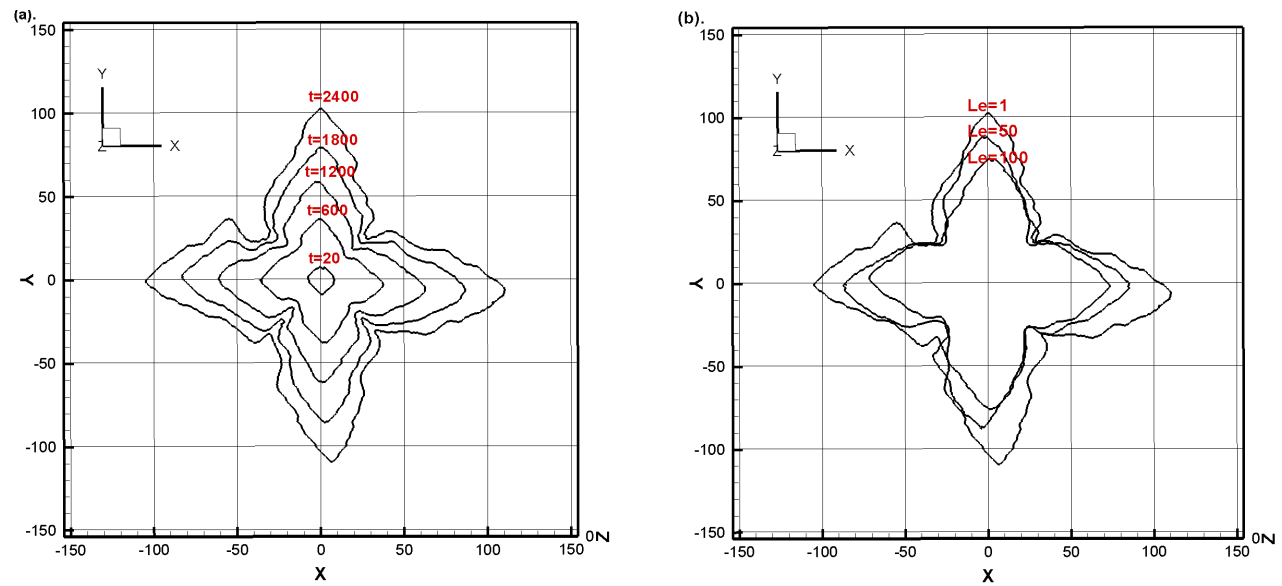

Figure 5.4: Growth of fourfold symmetric crystal for different Le with $E_{N}=0.1 \mathrm{eV}$. (a) Sequence of interface shapes shown at various time instants when the Lewis number $L e=1$. (b) The effect of Le on crystal growth at time $t=2400$.

$L e=1$ compared to the other three values for Le. Fig. 5.6 (b) illustrates the ratio of the number of solid atoms and surface atoms against time. The result shows that the number of surface atoms increases when the number of solid atoms is higher. The ratio of surface atoms to internal atoms is higher for small Lewis numbers, that is, higher surface area to volume ratio. It appears that the solute concentration becomes higher with decreasing Lewis number and thus more atoms are able to solidify. This is due to the diffusion of solute becoming faster.

In Figure 5.7 we show the development of the temperature fields ((a1), (b1), (c1)), concentration fields ((a2), (b2), (c2)) and surface images ((a3), (b3), (c3)) at three different times of the same simulation when $L e=25$. As might be expected, the thickness of the thermal boundary layer is seen to be much wider than the solute boundary layer when $L e$ is increased. This is because the value of thermal diffusivity is larger than diffusivity of the solute in both phases. Figures 5.7 (a3), (b3) and (c3) show the surface images of the crystal at times $t=400,1400$, and 2600. Figure 5.7 (a3) corresponds to 40049 atoms which are coloured according to their coordination 


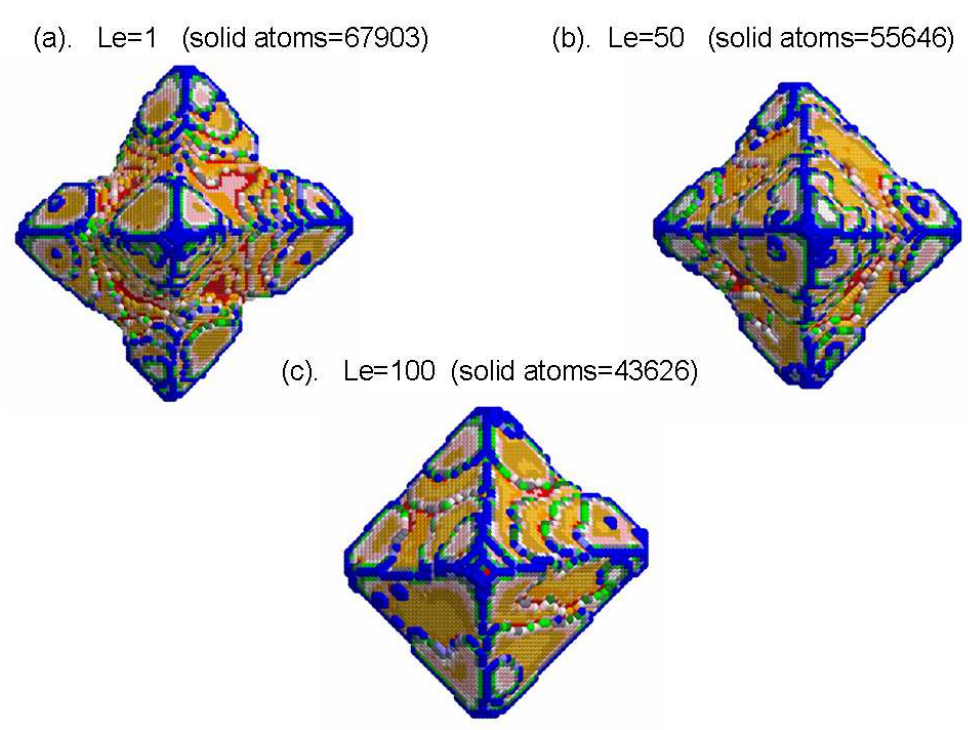

Figure 5.5: Three surface images of three separate simulations taken from the same time $(t=540)$ and coloured by their coordination number $N_{i j k}$. (a) for $L e=1$, containing of 67903 atoms, (b) for $L e=50$, containing of 55646 atoms, and (c) for $L e=100$, containing of 43626 atoms.

number $N_{i j k}$ (blue colour for high values of $N_{i j k}=1,2$ and red color for low values of $N_{i j k}=10,11$ ). Figure 5.7 (b3) and (c3), the solidifying atoms (that is, liquid atoms on the surface) are coloured using the heat flux $J_{i j k}$ and correspond to 304488 (b3) and 986291 (c3) atoms.

Figure 5.8 shows the temperature fields ((a1), (b1), (c1)), concentration fields ((a2), (b2), (c2)) and surface images ((a3), (b3), (c3)) at the nondimensional time of 2400 for the situations when $L e=1,20,50$. The thickness of the thermal and solute boundary layer are slightly similar when $L e=1$ as is indicated in Figure 5.8 (a1) and (a2). Note the thermal boundary layer is similar to all values of the Lewis number at a particular time but the solute boundary layer is different. If $L e$ is decreased (see Fig. 5.8 (b2) where $L e=20)$ the rate of the solute diffusion toward the interface increases and the depletion of solute along the solid-liquid interface is reduced. On the other hand, if $L e$ is increased (see Fig. 5.8 (c2) where $L e=50$ ) the rate of 

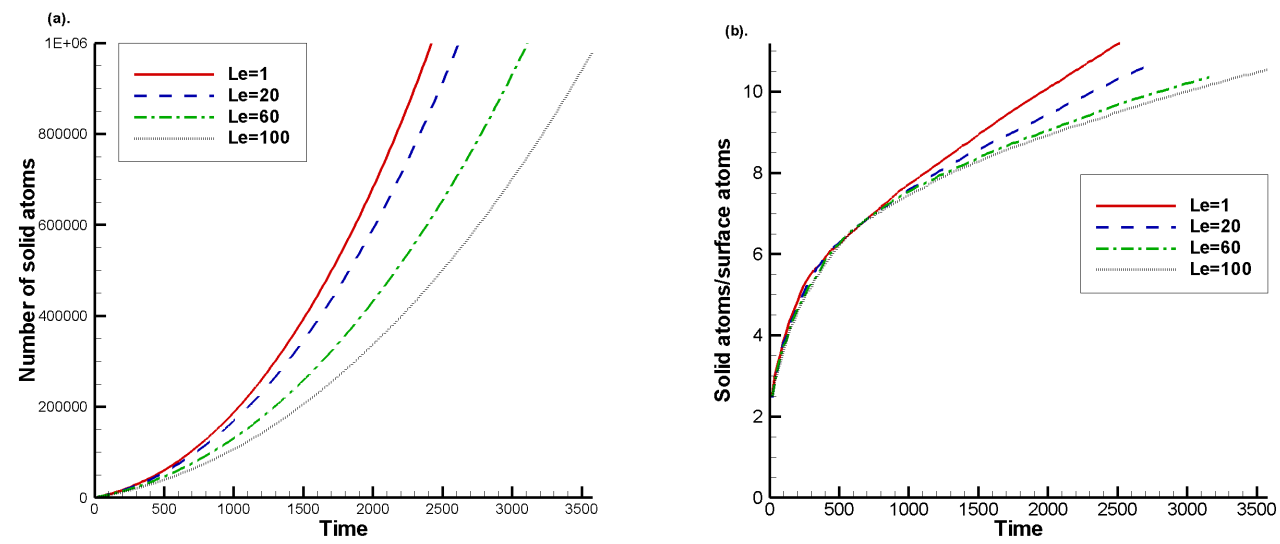

Figure 5.6: Comparison of the Lewis numbers when $E_{N}=0.1 \mathrm{eV}$ is the same in all simulations. The first plot (a) shows the number of solid atoms and the second plot (b) illustrates the ratio of the number of solid atoms and surface atoms as a function of time. The solid line indicates the $L e=1$ for dimensionless $\kappa$ and $D$. The dashed line shows the result of using $L e=20$, the dashdotdotted and dotted lines are the results obtained by using $L e=60,100$.

solute diffusion is reduced and the interface solute gradient increases.

As noted above, for $L e \rightarrow 0$ the diffusion of solute toward the interface is getting faster and produces a thicker solutal boundary layer in the liquid. At the opposite extreme, as $L e \rightarrow \infty$ the rate of solute diffusion toward the interface is very slow and produces a thinner solutal boundary layer in the liquid especially near the upstream tip. Figures $5.9(b, c, d)$ show the values of the contours indicated that the solute depletion in the grooves between the arms is higher than at the tip of the dendrite. Thus, the atoms located near the grooves are likely to find themselves in a pool of lower attachment rate than those which are approached and engulfed by the tip. Visual inspection of Figure 5.9 (a) identified the presence of thermal gradients near the interfaces. As expected, the gradient between the liquid and solid phases is strongest near the growing dendrite tips where the velocity is greatest. It is weaker along the nearly stationary initial in- 

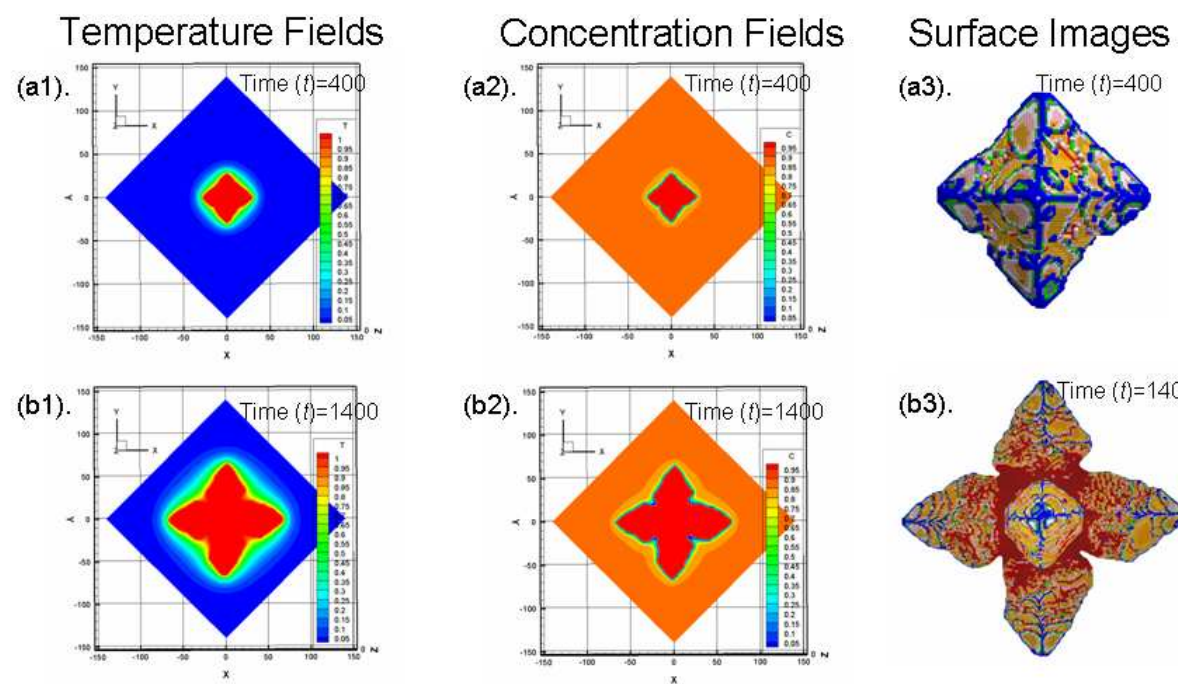

(b2).

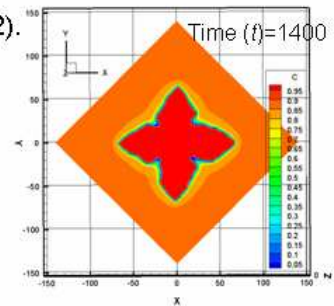

(c2).

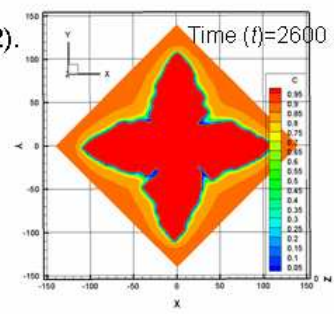

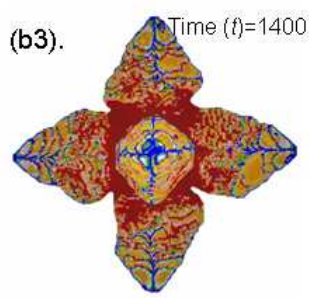

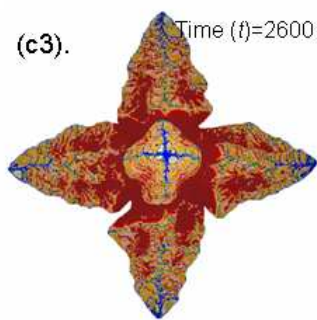

Figure 5.7: Temperature fields ((a1), (b1), (c1)), concentration fields ((a2), (b2), (c2)) and surface images ((a3), (b3), (c3)) of a single simulation at three different times, $L e=25$. Images at row one (a1), (a2) and (a3) are taken from the early stages at $t=400$. At row two images (b1), (b2), and (b3) are taken at $t=1400$ and the snap shot images at row three (c1), (c2) and (c3) are taken from the late stages when $t=2600$.

terface.

It can be seen that the simulation results in the present model give a very good image of crystal growth compared to other images of past studies (see Figure 5.10). Fig. 5.11 shows the development of a dendrite and its sidebranches when $L e=1$. These images correspond to four different times in the same simulation that started from a spherical cluster of about 500 atoms. The first two images (top) were taken from the early stages, showing the solid atoms that lie on the surface of the crystal and 
Temperature fields

(a1).

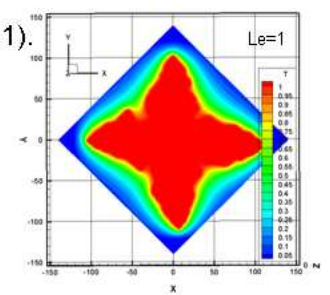

(b1).

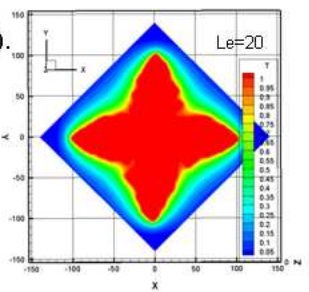

(c1

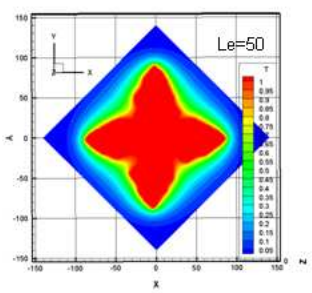

Concentration fields

(a2).

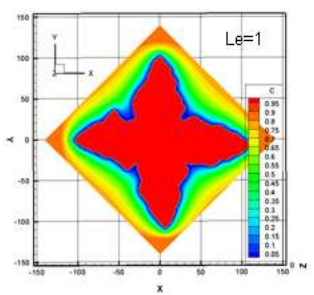

(b2).

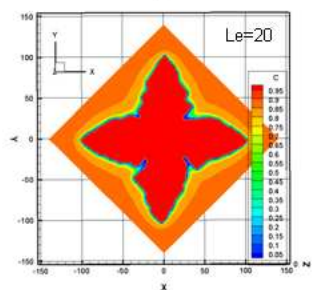

(c2)

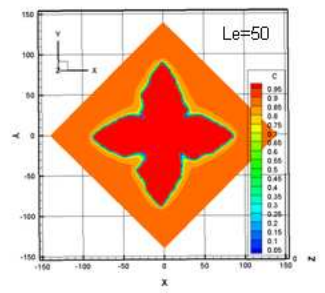

Surface images

(a3).

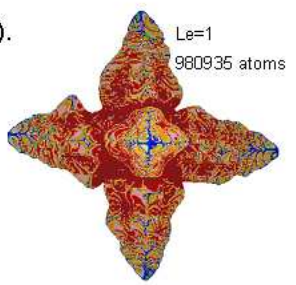

(b3).

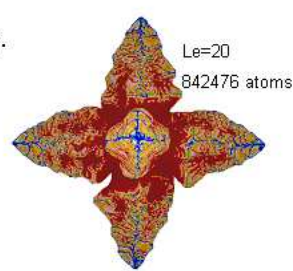

(c3).

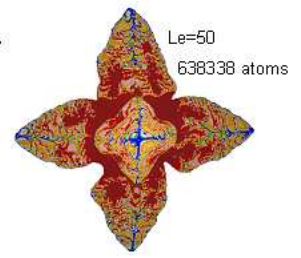

Figure 5.8: Temperature fields ((a1), (b1), (c1)), concentration fields ((a2), (b2), (c2)) and surface images ((a3), (b3), (c3)) of three separate simulations at identical time $(t=2400)$. The images at row one are taken when $L e=$ 1 and correspond to 980935 atoms. At row two images are taken when $L e=20$ and corresponding to 842476 atoms. The last row images are taken when $L e=50$ and correspond to 638338 atoms. Images (a3), (b3), and (c3) are coloured according to temperature gradient.

are coloured according to their coordination numbers. All the simulations in the current chapter have used the value of $K=10^{6}$, so that surface diffusion dominates, especially during the early stages of growth when the surface area being small. This early stages indicates the crystal grows close to its equilibrium Wulff shape. But then at late-times surface diffusion is less important as the shape is far from the Wulff shape.

Figure 5.11 (a) shows that the truncated octahedral shape can be understood as a competition between the two slowest growing facets, the (100) 

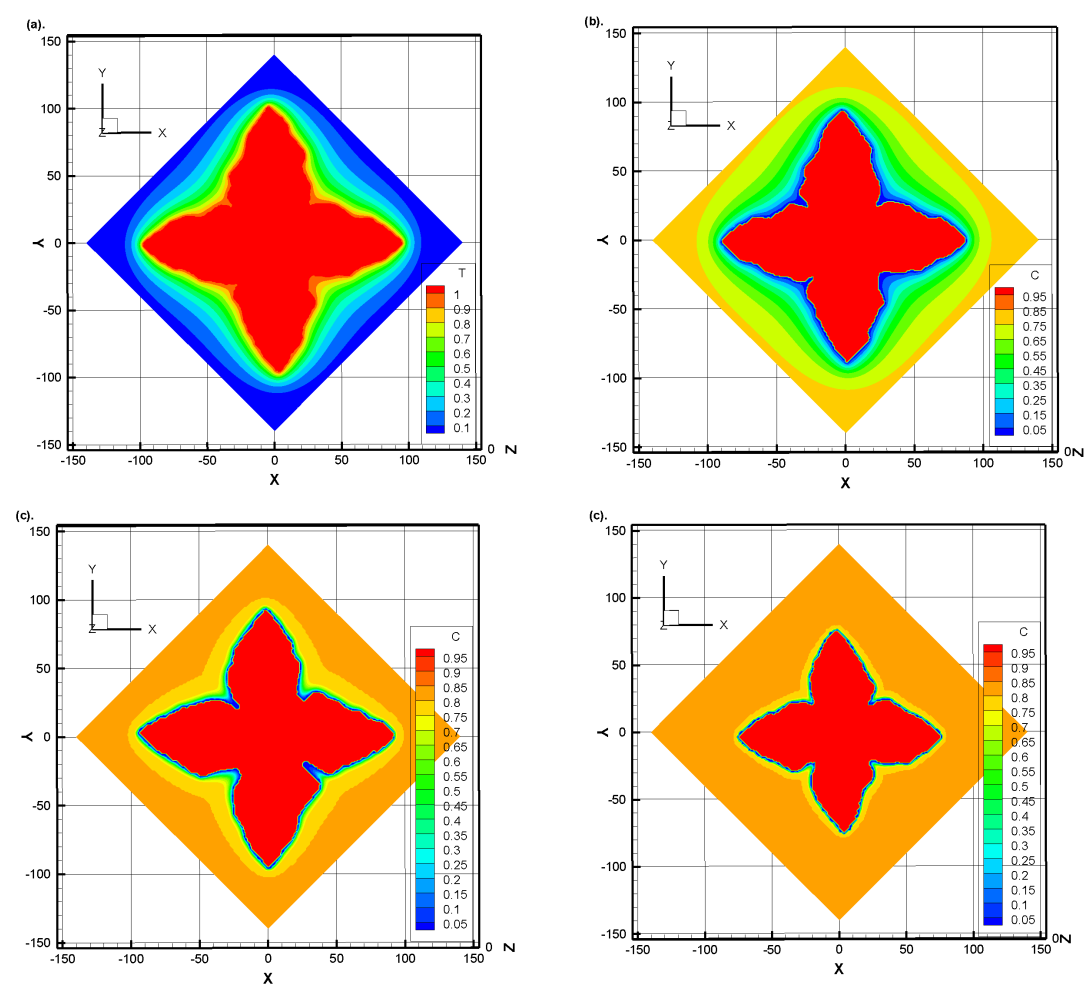

Figure 5.9: $(\mathrm{a}, \mathrm{b})$ shows the temperature and concentration fields when $L e=1$ at $t=2200$. (c, d) shows the concentration fields when $L e=20$ and $L e=100$ at $t=2200$.

and (111) facets, where the diffusing atoms have 4 and 3 nearest neighbours, respectively. Note that for an equal number of displayed lattice sites, there would be a net flux of atoms from a (111) facet to a (100) facet, due to the faster hopping rate on the former. This result tends to favour nucleation on the (100) facet rather than the (111) facet. During growth, the surface is nearly isothermal, with $T \approx T_{M}$. The isotherms near protruding regions of the surface become compressed as the crystal becomes bigger. The compression is very noticeable at the vertices of the octahedral structure [101]. This effect, which implies a steeper temperature gradient, enhances nucleation, and we can see in Figure 5.11 (b) that there is a cascade of steps that starts to flow away from the vertices. As the crystal be- 

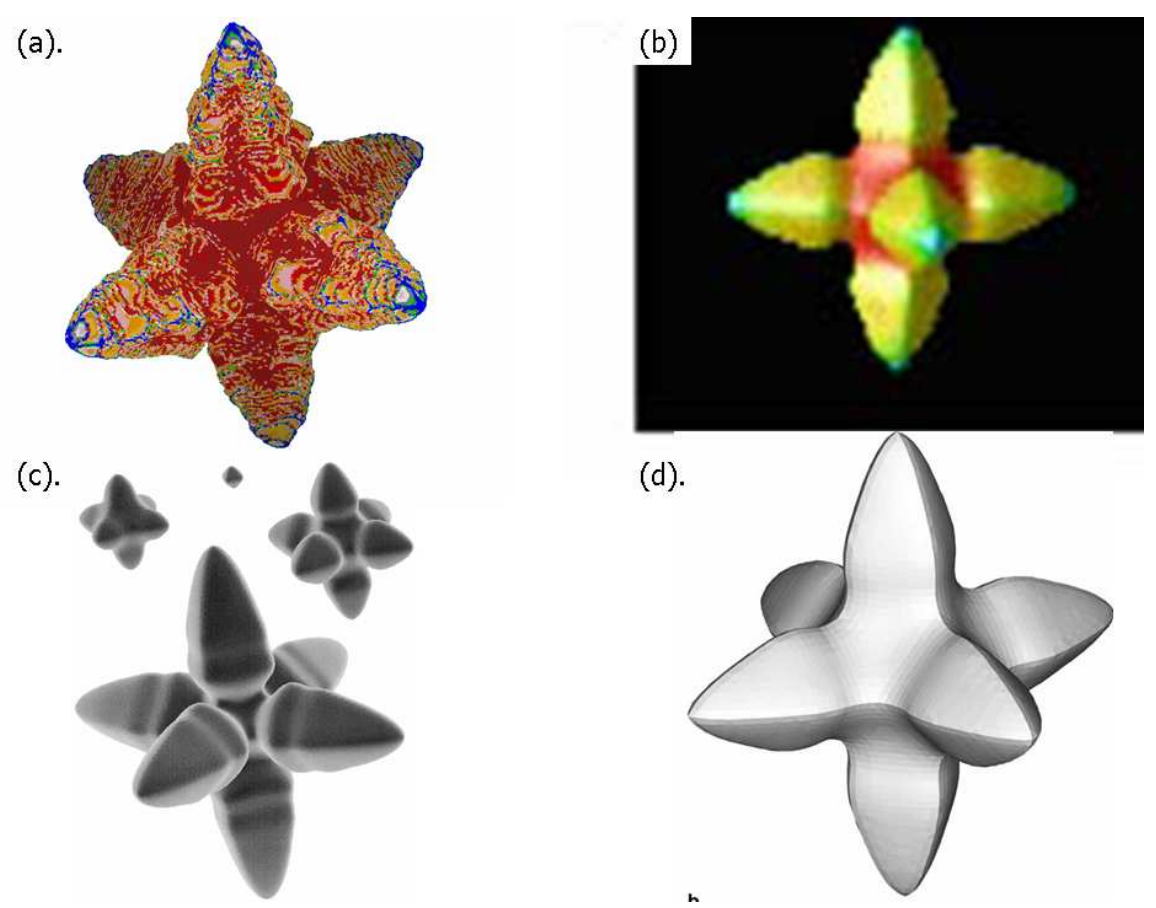

Figure 5.10: Image (a), snapshot of a dendrite shape from the current model. Image (b), snapshot of a dendrite shape using phase-field models [157]. Image (c). snapshots of a dendrite shape using multiscale random-walk algorithm [5]. Image (d), snapshot of a dendrite shape using phase-field model [139].

comes larger, this effect begins to change the morphology of the crystal. In particular, note that the edges are no longer straight in Figure 5.11 (b). The last two images (bottom (c) and (d)) in Figure 5.11 correspond to much later stages of the simulation and show the liquid atoms on the surface which are coloured according to the temperature gradient. These images also show the morphological instability which leads to dendritic growth. Figure 5.12 shows three surface images of two different simulations at the same time step later in the simulation for a case where $L e=1$ and 100. The solute accumulation in the grooves between the arms is thicker in Figure 5.12 (b). This can be seen in the illustration by the fact that more facet regions can be seen in the grooves of the structure (see Figure 5.12 (b)) 

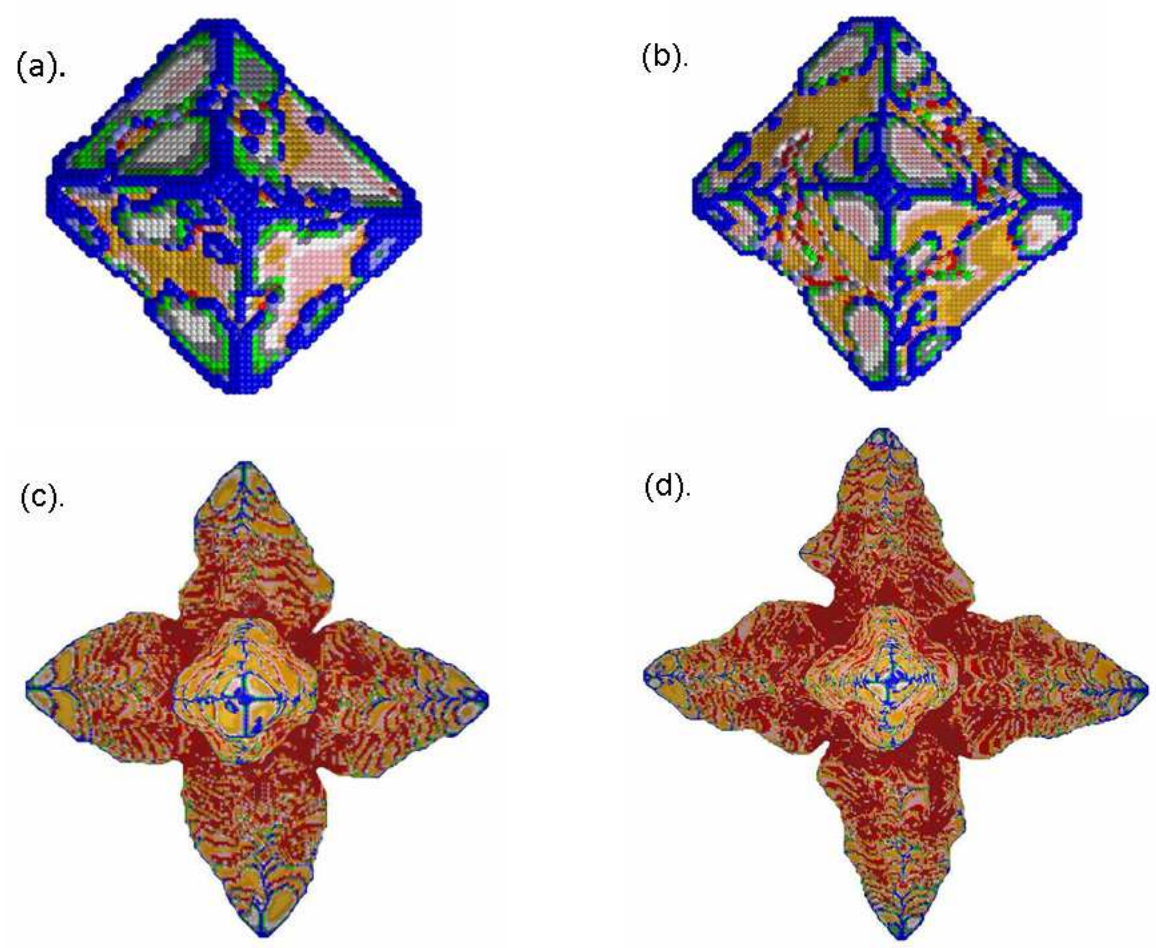

Figure 5.11: Four surface images of a single simulation at four different times when $L e=1$ and $E_{N}=0.1 \mathrm{eV}$. The top images (a) and (b) are from the early stages and correspond to the stage at which the crystal contains about $10^{3}$ (left) and $10^{4}$ (right) atoms coloured according to coordination number $N_{i j k}$. The bottom images (c) and (d) are taken from the late stages, showing the crystal containing about $10^{5}$ (left) and $10^{6}$ (right) atoms coloured according to the temperature gradient.

compared to the later stages in Figure 5.12 (a). The last image (Figure 5.12 (c)) shows what the crystal looks like in three dimensions.

\subsubsection{Mass conservation}

An additional means of checking that acceptable numerical solutions are being obtained is to examine the overall conservation of heat or solute, and therefore, in these initial calculations the global mass balance (that is, the conservation of solute) was frequently examined. This implies that 


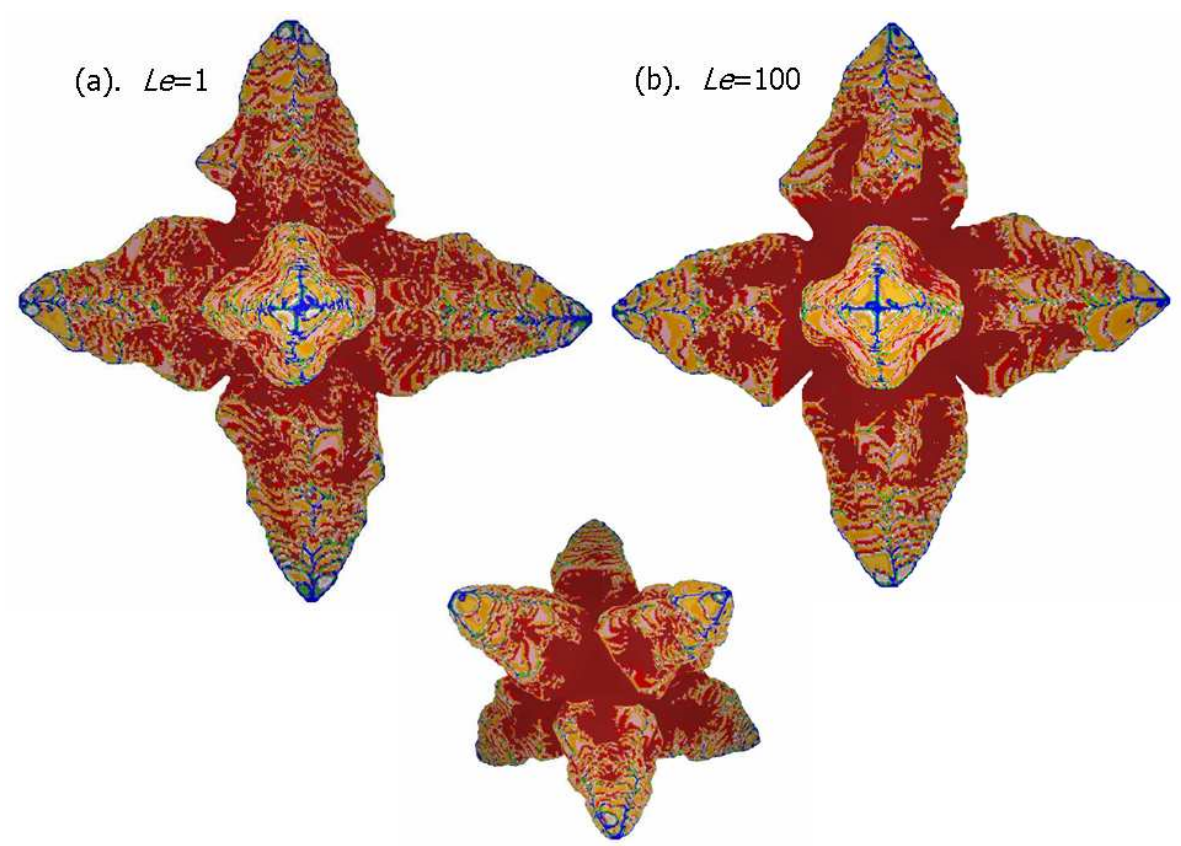

(c). Crystal looks like in three dimensions

Figure 5.12: Three surface images of two different simulations at the same time $\mathbf{t}=2600$ from the late stages. Image (a) shows the surface image when $L e=1$ and images (b) and (c) illustrate the surface images when $L e=100$. Image (c) shows what the crystal look like in three dimensions. All images are coloured by temperature gradient.

the number of atoms per unit time crossing into the simulation domain at any given point in time should equal the difference between the number of atoms per unit time at that specific point and that of the immediately preceding time unit inside the simulation domain. This is necessary for the solute to be conserved. We have introduced the boundary conditions discussed in Section 5.3 so that mass is approximately conserved. The flux is calculated as:

$$
\frac{\Delta N}{\Delta t}=\frac{1}{L e} \int\left(\frac{\partial C}{\partial \hat{s}} \cdot \widehat{\mathbf{n}}\right) d S,
$$

where $N$ is the number of atoms, $\widehat{\mathbf{n}}$ is the normal unit vector which is perpendicular to the surface $S$, and $d S$ is the differential surface element. 
The difference in the number of atoms at a particular time is given by

$$
\text { Atom }_{t_{i}}-\text { Atom }_{t_{i-1}}=\left(L_{\text {Atom }_{t_{i}}}+S_{\text {Atom }_{t_{i}}}\right)-\left(L_{\text {Atom }_{t_{i-1}}}+S_{\text {Atom }_{t_{i-1}}}\right)
$$

where Atom $_{t_{i}}$ is the total number of atoms of the current time interval, Atom $_{t_{i-1}}$ is the total number of atoms of the previous time interval, $L_{\text {Atom }_{t_{i}}}$ and $S_{\text {Atom }_{t_{i}}}$ are the number of liquid and solid atoms of the current time interval. Figure 5.13 shows the fitted graph of the values of flux with the atom difference as a function of time. As it appears from Figure 5.13, the fitted graph is still not apparent that mass is conserved on average on longer timescales. The high fluctuation of the mass difference is due to the influence of the random number and average velocity during the simulation.

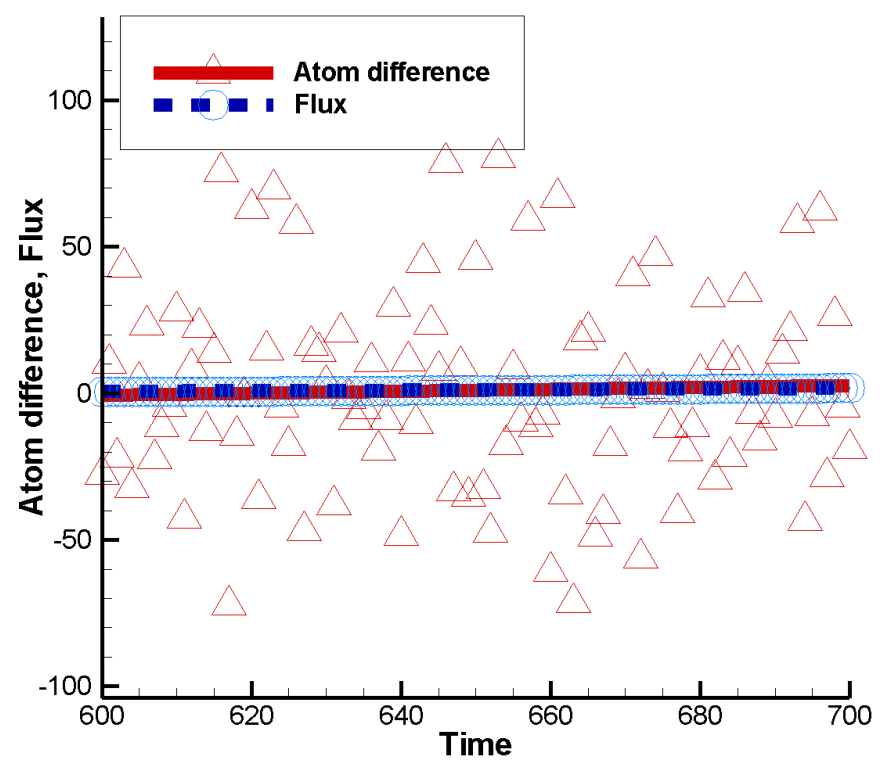

Figure 5.13: Point of total flux $(\circ)$ and atom difference $(\triangle)$ as a function of time. The dashed and solid lines indicate the best fitted lines of the total flux and atom difference. 


\subsubsection{Tip Velocity}

The tip velocity is measured by identifying the average height of the maximum tip of the interface and its neighbors as a function of time, similar to what was described in references [160,161]. The dimensionless slope of the liquidus is set to $m=0.0045$ and the value of $S^{-1}=0.9$ with the Lewis number set to $L e=1$. When $L e$ is increased, the tip velocity and the maximum height are expected to decrease due to the solute diffusion rate decreasing. In this case, we varied the value of $S t^{-1}$ within a realistic range of values, while staying under the hypercooling threshold $S t^{-1}<1$ in order to boost the attachment rate of atoms for each value of $L e$. Figure 5.14 (a) shows the tip velocity plotted against time for the upward, downward and horizontal growing dendrite arms. The gradient of solid, dashed and dash-dot-dot lines can be seen to approach a steady state shown by the dotted line. The dotted line indicates that the tip velocity of the dendrite arms decreases and approaches an asymptotic steady-state velocity $V=0.044$ in the later stages. The dendrite continues to grow and coarsen until the end of the run where it reaches the far side of the system. Figure 5.14 (b) shows the relationship between the tip velocity and the maximum height of the branches of the growing dendrite arms. As shown, the tip velocity is decreased when the maximum height of the interface increases. It can be seen that the primary branches of an equiaxed dendrite all begin growth close to one another and proceed to grow away from each other. Figure 5.14 (c) illustrates that the tip velocity is decreased when $L e$ increases and also that the number of time steps required to reach the final update is longer when $L e$ increases.

To validate the simulation, a comparison of tip velocity as a function of time with the predictions of other studies for crystal growth was carried out. It can be seen in Figure 5.15 (a) that Schulze's [101] and Tan et al.'s [139] results are found to agree with the Continuum-KMC Method when the $L e=1$. Schulze [101] considered the growth of a single FCC dendrite into an undercooled melt by adopting a discrete, atomistic model 

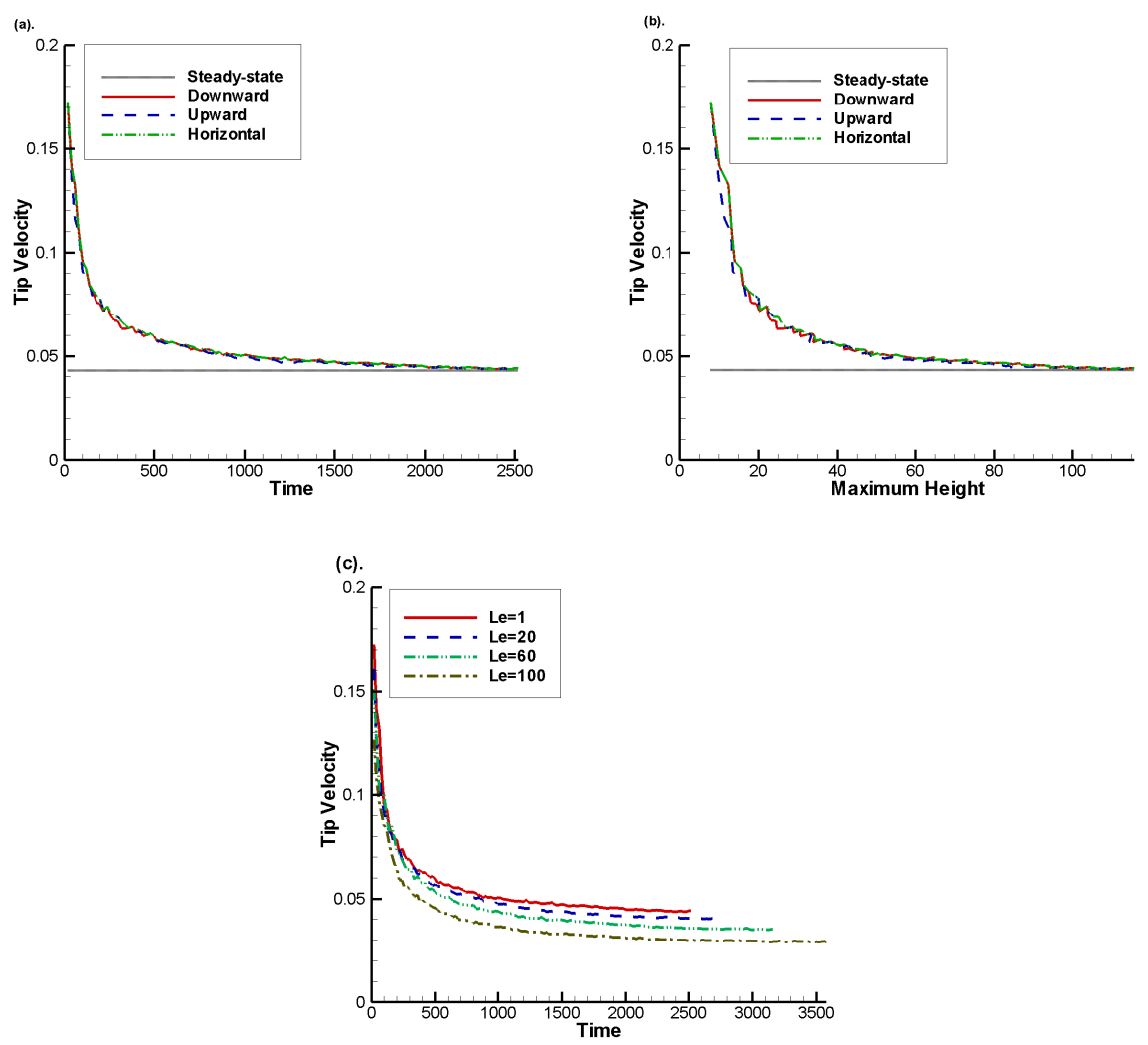

Figure 5.14: Plots (a) and (b) show tip velocity of the dendritic growth when $L e=1$ and $E_{N}=0.1 \mathrm{eV}$. (a) Dimensionless tip velocity of the dendritic growth plotted against time. (b) Dimensionless tip velocity vs maximum height. Plot (c) shows tip velocity as a function of time of different values of $L e$.

in the spirit of the kinetic Monte Carlo method. Tan et al.'s [139] method combining features of front-tracking and fixed-domain methods based on the level set method is presented to model dendritic solidification of pure materials. Using a slightly higher undercooling of 0.65 , the steady dendrite tip velocity increases to about 0.047 . Their result is very close to our steady-state velocity of 0.044 . We also found that our results (tip velocity against time) when $L e=1000$ agree with the predictions of Karma [162] shown in Figure 5.15 (b). Karma considered a Phase-Field formation to 

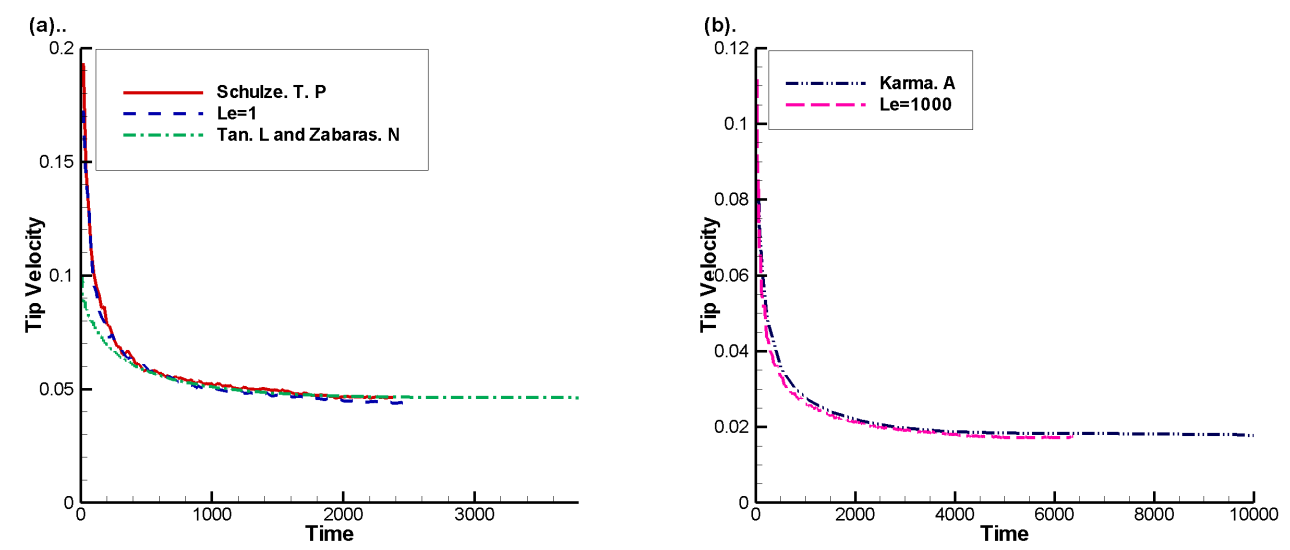

Figure 5.15: Comparison of tip velocity with Schulze result. $E_{N}=0.1 \mathrm{eV}$ is the same in all simulations.

simulate quantitatively microstructural pattern formations in an alloy.

\subsubsection{Anisotropy}

As described above, the crystalline anisotropy effect is included in the model as a surface diffusion process. Note that $E_{N}$ is not anisotropy by itself but it just leads to anisotropy with the help of the surface diffusion parameter $K$ which has been adjusted to exhibit different characteristic morphologies. Therefore, surface anisotropy depends on $E_{N}$. It can be seen that the value of the maximum height increases faster when the $E_{N}=0.11 \mathrm{eV}$ than the rest of the $E_{N}$. Again, the growth proceeds from an initial spherical cluster of 500 atoms and the sidebranches are expected to grow along the principal crystalline directions. Figure 5.16 (a) shows the maximum height plotted against time for growing dendrites in four different values of surface anisotropy. Figure 5.16 (b) shows that when the anisotropy is decreased, the interface velocity reduces and thus the computational domain size and simulation time increase substantially. The tip curvature for the high anisotropy crystal is much higher than the low anisotropy crystal. Also the concentration and thermal boundary layers 

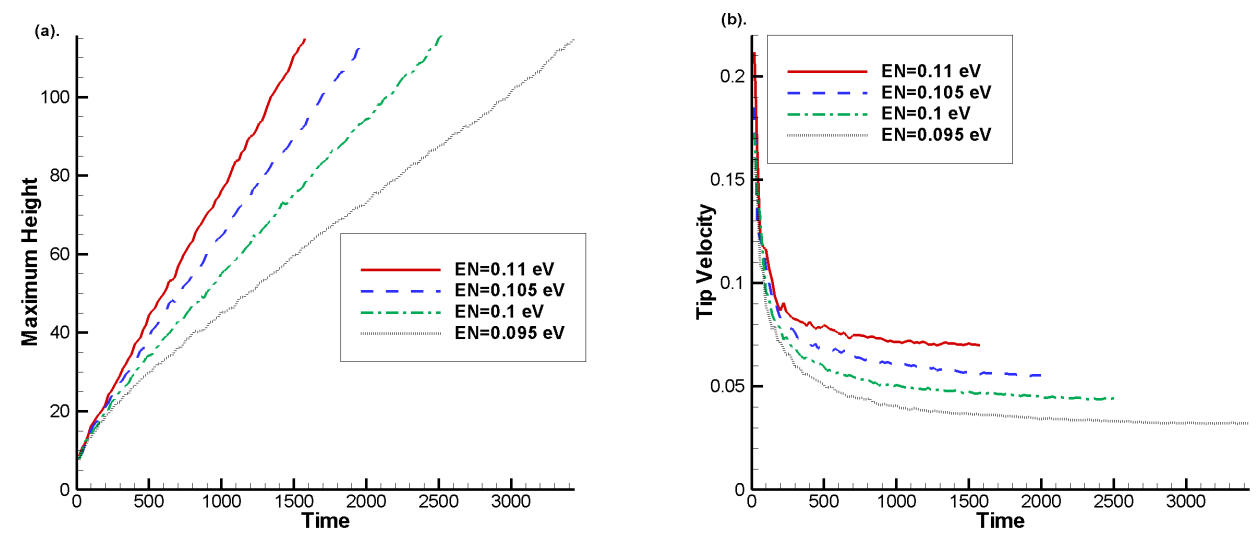

Figure 5.16: Tip velocity and maximum height of the dendritic growth when $L e=1$ for different values of $E_{N}$. (a) Dimensionless maximum height of the interface as a function of time. (b) Dimensionless tip velocity of the dendritic growth plotted against time.

in the latter case are shallower than for the previous high anisotropy case. When the anisotropy is lower than $0.1 \mathrm{eV}$, it can be seen that the tip region is smooth and the crystallite becomes fully faceted. As the anisotropy becomes larger than $0.1 \mathrm{eV}$, discontinuity in the variation of interface orientation occurs due to the presence of missing orientations, and therefore the smooth parabolic dendrite becomes unstable, resulting in the formation of corners at the tips of the main stem and sidebranches of the dendrite.

Figure 5.17 indicates the development of four surface images of a single simulation at four different times when $E_{N}=0.13 \mathrm{eV}$. The top images (a) and (b) are taken from the early stage showing the solid atoms, coloured by coordination number $N_{i j k}$, that lie on the surface of the crystal. In the first image (a), the crystal contains 5311 atoms and in the second (b), there are 48693 atoms. The bottom images (c) and (d) are taken from the late stages indicating the solidifying atoms (that is, liquid atoms on the surface) are coloured using the heat flux. The images correspond to the stage at which the crystal contains 315980 on the left (c) and 1026615 atoms on the right (d). These images show clearly that the branching process occurs 
at earlier stages of growth if the surface diffusion is less active.
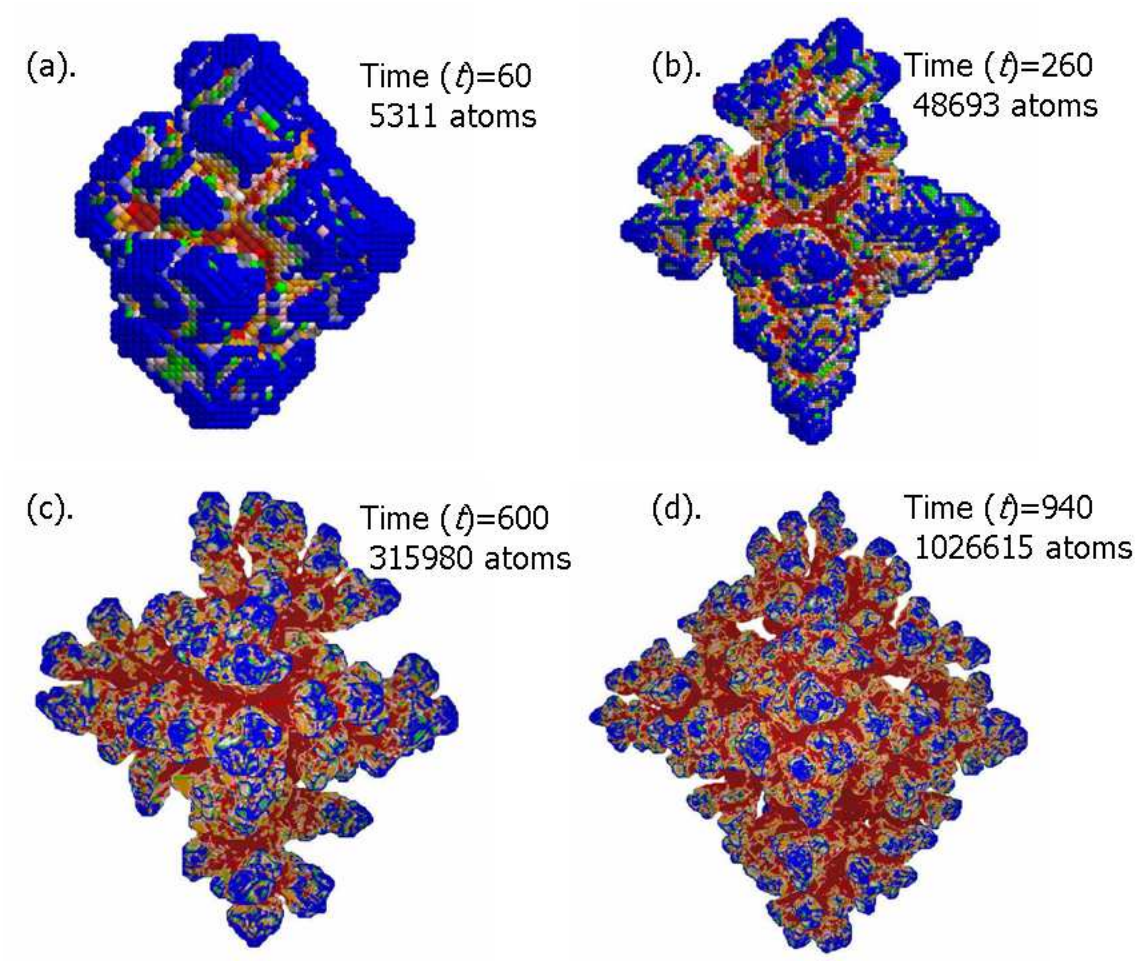

Figure 5.17: Four surface images of a single simulation at four different times $(t=60,260,600,940)$ when $E_{N}=0.13 \mathrm{eV}$. The top images (a) and (b) are from the early stages, showing 5311 (a) and 48693 (b) atoms coloured according to coordination number $N_{i j k}$. The bottom images (c) and (d) are taken from the late stages, showing 315980 (c) and 1026615 (d) atoms coloured according to the temperature gradient.

Figure 5.18 shows the images from the later stages of several different simulations when the value of $E_{N}$ is varied to exhibit four characteristic morphologies. The colours show the distribution of the heat flux $J_{i j k}$ in the liquid atoms on the surface. When the surface anisotropy starts to increase, the branching process occurs at an earlier stage of the crystal growth due to surface diffusion being less active. The formation of secondary branches, their growth and competition, and finally their coarsening have already formed when the crystal is about the same size as that in 
the early state of Figure 5.11 (a). Most of the tertiary branches developed only on the side of the secondary branch facing the primary dendrite tip, which is consistent with the experimentally observed features. At about $E_{N}=0.15 \mathrm{eV}$, the dendrite loses its octehedral symmetry and takes on a cauliflower-like appearance shown in Figure 5.18 (d).

(a). $E N=0.095 \mathrm{eV}$

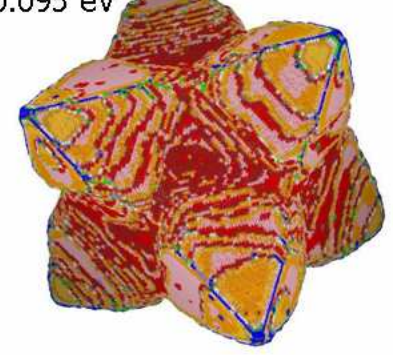

(c). $E_{N}=0.125 \mathrm{eV}$

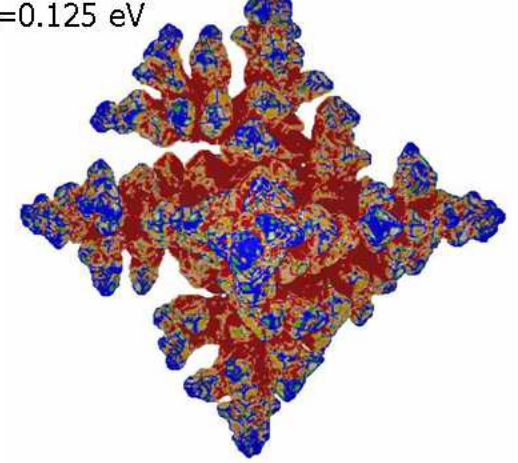

(b).

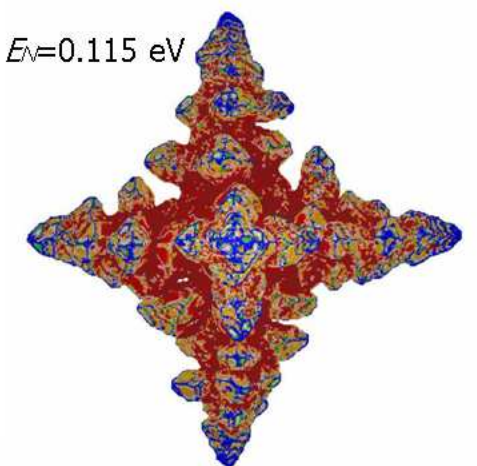

(d). $E N=0.15 \mathrm{eV}$

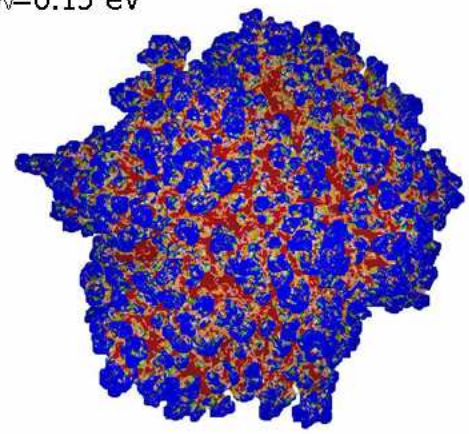

Figure 5.18: Four surface images from the late stages of four separate simulation, showing about $10^{6}$ atoms coloured according to the temperature gradient. $E_{N}=(0.095,0.115) \mathrm{eV}$ (top images (a) and (b)) and $E_{N}=(0.125,0.15) \mathrm{eV}$ (bottom images (c) and (d)). Le $=1$ is the same in all simulations.

\subsubsection{Multicrystal growth}

The current method can also be used to model the growth of multiple seeds. Note that this is something that is hard to do with front tracking 
methods such as those used by Tan et al. [139]. In Figure 5.19 we show the development of the temperature fields ((a1), (b1), (c1)), concentration fields ((a2), (b2), (c2)) and surface images ((a3), (b3), (c3)) at three different times of the single simulation when $L e=1$. As might be expected,

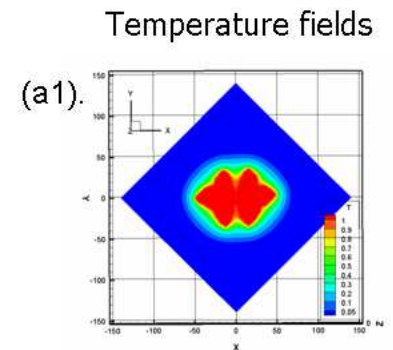

(b1).

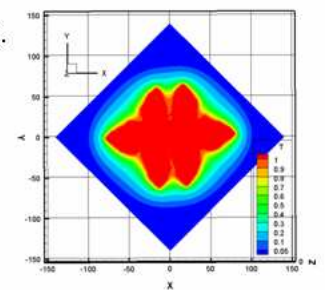

(c1).

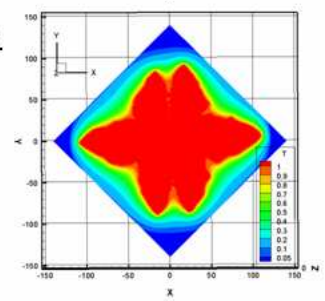

Concentration fields

(a2).

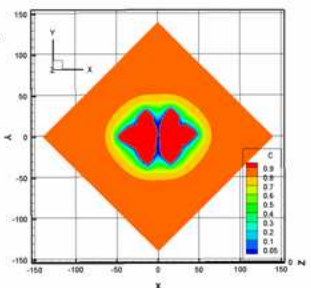

(b2).

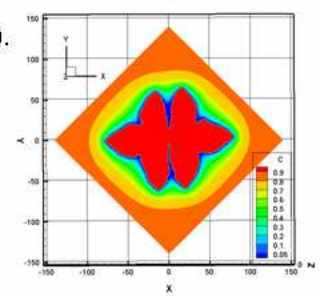

(c2).

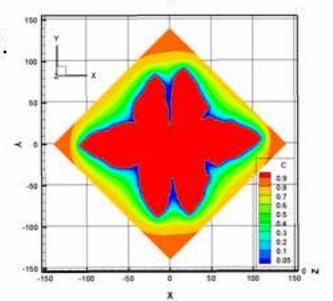

Surface images

(a3).

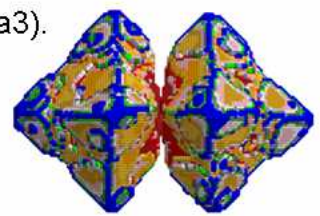

(b3).

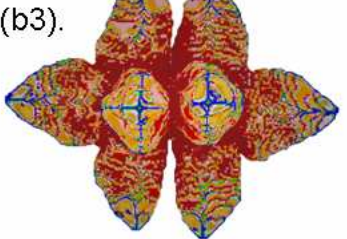

(c3).

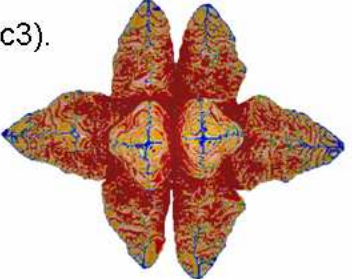

Figure 5.19: Temperature fields ((a1), (b1), (c1)), concentration fields ((a2), (b2), (c2)) and surface images ((a3), (b3), (c3)) of a single simulation at three different times using $L e=1$. Images at row one (a1), (a2) and (a3) are taken from the early stages when $t=400$. At row two images (b1), (b2), and (b3) are taken when $t=1400$ and the snap shot images at row three (c1), (c2) and (c3) are taken from the late stages when $t=2200$.

the thickness of the thermal and solute boundary layers are slightly similar as indicated in Figure 5.19 column one and two. This indicates that the value of thermal diffusivity is similar to the value of the solute diffusivity. Figures 5.19 (a3), (b3) and (c3) show the surface images of the crystal at times $t=400,1400$, and 2200. Figure 5.19 (a3) correspond to 
(a). Time $(t)=100$

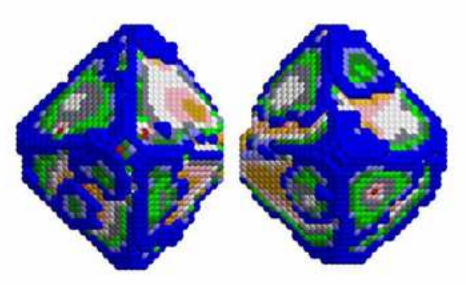

(c.). Time $(t)=1500$

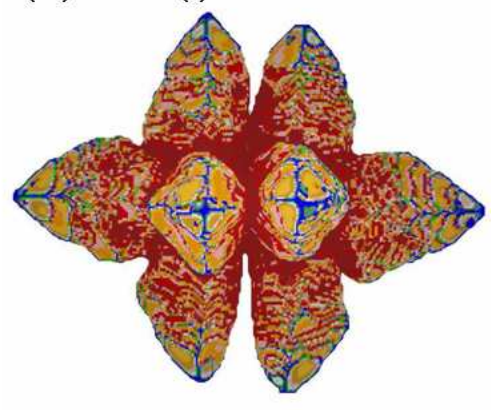

(b). Time $(t)=440$

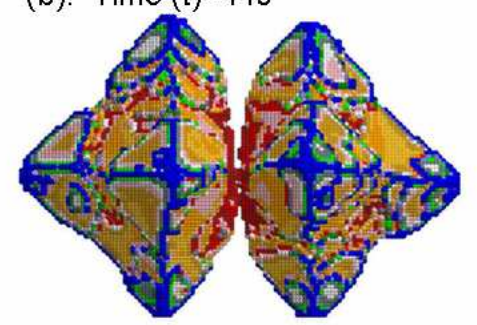

(d). Time $(t)=2320$

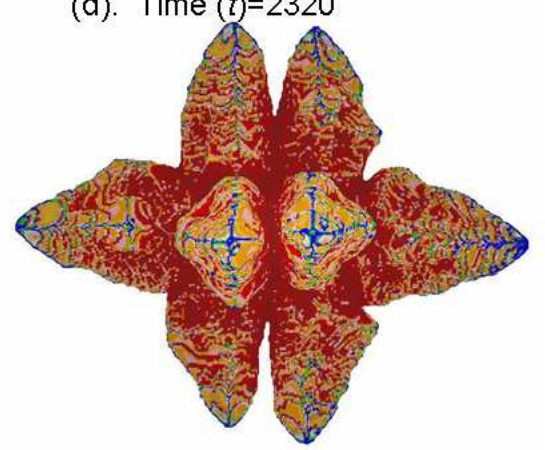

Figure 5.20: Evolution of two identical seeds of a single simulation at four different times $(t=100,440,1500,2320)$ when $E_{N}=0.1 \mathrm{eV}$. The top images (a) and (b) are taken from the early stages and coloured by coordination number. The bottom images (c) and (d) are taken from the late stages and coloured according to temperature gradient.

66269 atoms and coloured according to the coordination number $N_{i j k}$. Figure 5.19 (b3) and (c3), the solidifying atoms (that is, liquid atoms on the surface) are coloured using the heat flux $J_{i j k}$ and correspond to 454223 (b3) and 1025363 (c3) atoms.

Figure 5.20 indicates the development of two identical seeds in a single simulation at four different times when $E_{N}=0.1 \mathrm{eV}$. It can be seen that the growth of the main arms is suppressed by the nearby dendrite especially for the main arms that are facing each other. The interfaces of these arms were allowed to merge when they come in contact. As solidification proceeds, growing and coarsening of the primary trunks occurs, to- 
(a)

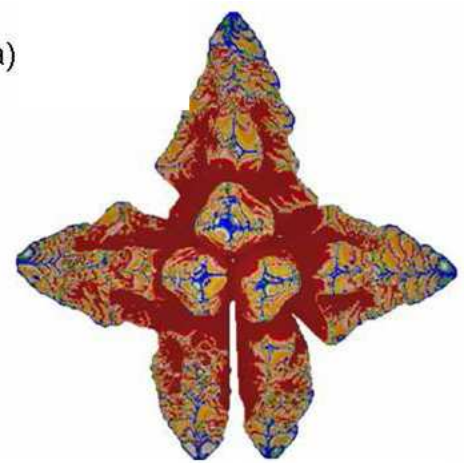

(c)

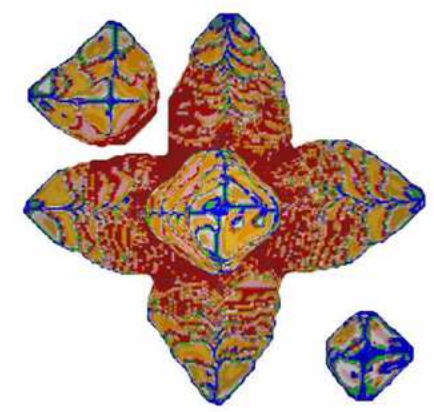

(b).

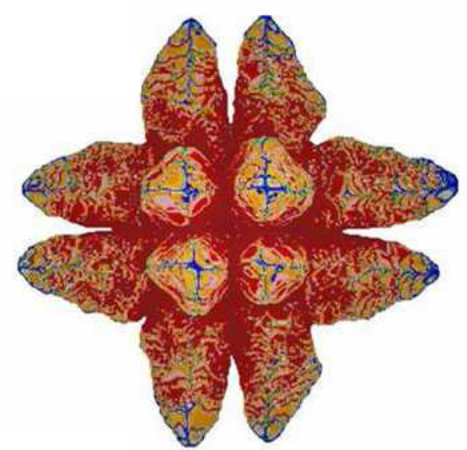

(d).

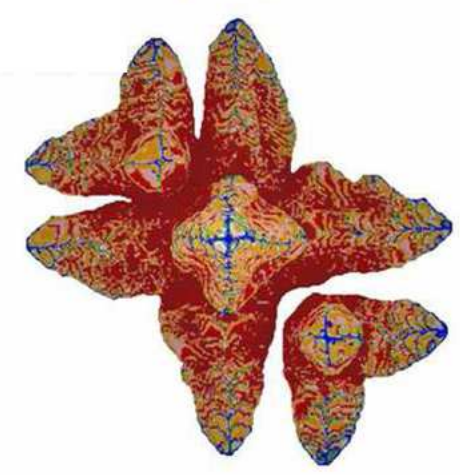

Figure 5.21: Interactions in the solidification of several dendrites. (Top images (a) and (b)) Two surface images from the late stages of two separate simulations of three and four identical seeds growing at the same time. (Bottom images (c) and (d)) Two surface images of three seeds are growing in different times in a single simulation. All images were coloured according to temperature gradient. $L e=1$ and $E_{N}=0.1 \mathrm{eV}$ are the same in all simulations.

gether with the branching and coarsening of the smaller secondary arms. Figure 5.21 (top) shows three and four identical seeds with randomly assigned preferred growth orientations placed in the domain at the same time. Note that the interfaces naturally merge when they come into contact. Figure 5.21 (bottom) shows another case in which we place three seeds in the domain at different times. The second seed is placed in at a distance that allows the interfaces to merge when they come in contact but the third seed is not. Note that the new seeds are growing at the tem- 
perature and concentration fields of the sites in which they are initially placed. It can also be noted that when the seeds are placed further apart, the merging that is observed when the seeds are placed close together does not occur. There is a low attachment rate for the solidifying atoms as the concentration is depletes between the surfaces at the two developing crystals. This is because most of the regions on the surface between the two crystals are fully faceted and it is therefore difficult for atoms to deposit there. Note that these simulations were carried out without consideration of the dissolution process. The next section will include the dissolution process in the current KMC algorithm.

\subsection{Dissolution}

The change in crystal shape as a result of dissolution is a topic of interest for a wide range of materials including organics such as pharmaceuticals, proteins and specialty chemicals as well as inorganics in geology and semiconductors. The dissolution process not only affects the crystal shape but also plays a vital role in polymorphic phase transformations [163, 164]. Various works have previously discussed the possible end-shapes in crystal dissolution [165, 166, 167]. Moore [167] suggested that the shapes of mineralogical crystals result from faces becoming vertices and vertices becoming faces. Frank [165] proposed that crystals will disappear before changes in shape have ceased, and Gibbs suggested that shapes in crystal dissolution probably differ from that of theoretical equilibrium in a direction opposite to that of a growing crystal [168]. Despite these qualitative descriptions, Snyder et al. [163] developed a model that could quantitatively predict the evolution of crystal shapes during dissolution. Snyder et al.'s [164] findings present recently produced in situ experimental confirmation of the model's predictions for the dynamics of dissolving crystal shapes. Other works on crystal dissolution have traditionally been focused on the evolution of surface morphology in the immediate vicinity of 
isolated defects $[169,170,171]$. It is also necessary to include dissolution in order to study Ostwald ripening, that is when smaller crystals dissolve to the benefit of larger neighbouring crystals.

The potential occurrence of both dissolution and growth, can be a valuable mechanism for exposing crystal planes and generating crystal shapes that are not easily obtained through either growth or dissolution alone. The key difference between morphology evolution during growth and dissolution is that growth shapes tend to be dominated by slow growing planes, while dissolution shapes are dominated by fast moving planes [164]. A model for the shape evolution in dissolution is required in order to fully understand the effects of dissolution on the crystal shape. Thus, the current study aims to create a basic framework for such simulations using a face centered cubic lattice as an example. To achieve this objective, it is therefore necessary to develop kinetic Monte Carlo (KMC) models that are efficient for lattices that are large enough to contain a large number of atoms (defects) at realistic densities. The model developed in the current study provides significant insight into obtainable crystal morphologies, as well as demonstrating that crystals do not obtain immediate steady-state when dissolution is included (whereas they do in growth alone [172]). The model for shape evolution in growth and dissolution, in combination with the mechanisms for the appearance and disappearance of faces, can be applied to any crystal system. This model can predict and track each of the faces of a fully three-dimensional crystal during growth and dissolution. It requires a set of physical properties for implementation (dissolution rates and crystallographic information) as described in the following sections.

\subsubsection{Calculating the dissolution rate}

The present study examined the morphology of nanocrystal growth when the dissolution process is included. The mechanism for growth and dissolution of crystals varies depending on the classification of the crystal 
face [173]. As suggested by Cabrera and Levine [174] for calcite, the dissolution can be simply described as the inverse of nucleation and growth. For dissolution the same process occurs in reverse of our solidification process, with the solid atom being flipped and dissolved back into the melt as a liquid atom. The parameter values have been kept the same as our solidification process except for the value of $S t^{-1}=0.999$, which is increased within a realistic range of values while staying under the hypercooling threshold $S t^{-1}<1$. The condition is enforced in the simulations presented below by setting the dissolution rate equal to

$$
D_{q_{i j k}}= \begin{cases}J_{d}\left(\mathbf{x}_{i j k}\right), & \text { if } \sigma_{i j k}=1 \text { and } N_{i j k} \leq 3 \\ 0, & \text { otherwise }\end{cases}
$$

where

$$
J_{d}\left(\mathbf{x}_{i j k}\right)=S t^{-1} \frac{1}{12} \sum_{m=1}^{12}\left[T_{I}\left(\mathbf{x}_{i j k}\right)+T^{n}\left(\mathbf{x}_{i j k}+\mathbf{e}_{m}\right)\right],
$$

represents the net heat gain at site $\mathbf{x}_{i j k}$, measured in units of latent heat which is absorbed per atom upon dissolution. At best, this formula makes the stringent assumption that the dissolution involves very rough surfaces and far from equilibrium conditions. In the current model, the condition for dissolution is that a site must be occupied and must have less or equal to three solid neighbours.

\subsubsection{Comparing Growth and Dissolution}

To investigate the physical and mathematical models between the simulation using the growth and dissolution model and the growth model as discussed before, a comparison of the number of atoms as a function of time was carried out.

Figure 5.22 shows the comparison of the solid atoms and the tip velocity between two different simulations. The solid line indicates the growth 
alone and the dashed line shows the result of including the dissolution event. It can be seen from the graphs in Figure 5.22 (a), that more solid atoms were contained in the crystal at any particular time in the growth alone than in the growth and dissolution situation. This occurs due to the solid atoms being dissolved back into the melt as liquid atoms. The condition is that solid atoms can only dissolve if they have less than or equal to three solid neighbours. Many of the atoms having less than or equal to three neighbours can appear at the roughened faces especially at the edges and vertices, and roughened faces often have a curvature in directions without a strong bond chain. Figure 5.22 (b) shows the tip velocity
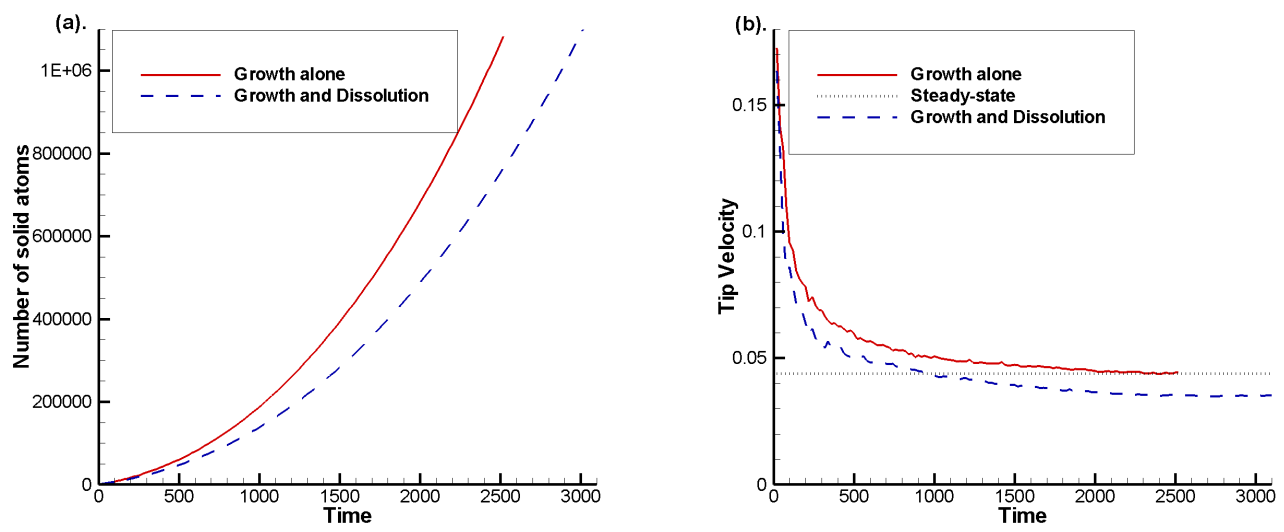

Figure 5.22: Comparison of the solid atoms and the tip velocity between two different simulations. The first plot (a) shows the number of solid atoms and the second plot (b) illustrates the tip velocity as a function of time. The solid line indicates the growth alone and the dashed line shows the result of including the dissolution event.

plotted against time for the horizontally growing dendrite arms in the situations of growth alone, and of growth and dissolution. The solid and dashed lines can be seen to be getting slower and slower as the time increased. The solid line indicates that the tip velocity of the dendrite arms decreases and they eventually reach a steady state shown by the dotted line. On the other hand, the dashed line demonstrates that the tip velocity 
does not obtain an immediate steady-state when dissolution is included. This is happening due to the fact that the dissolution process seems active at the edges and the tip. Note that most of the atoms on the rough surface have three neighbours or less lying on the surface edge and tip regions.
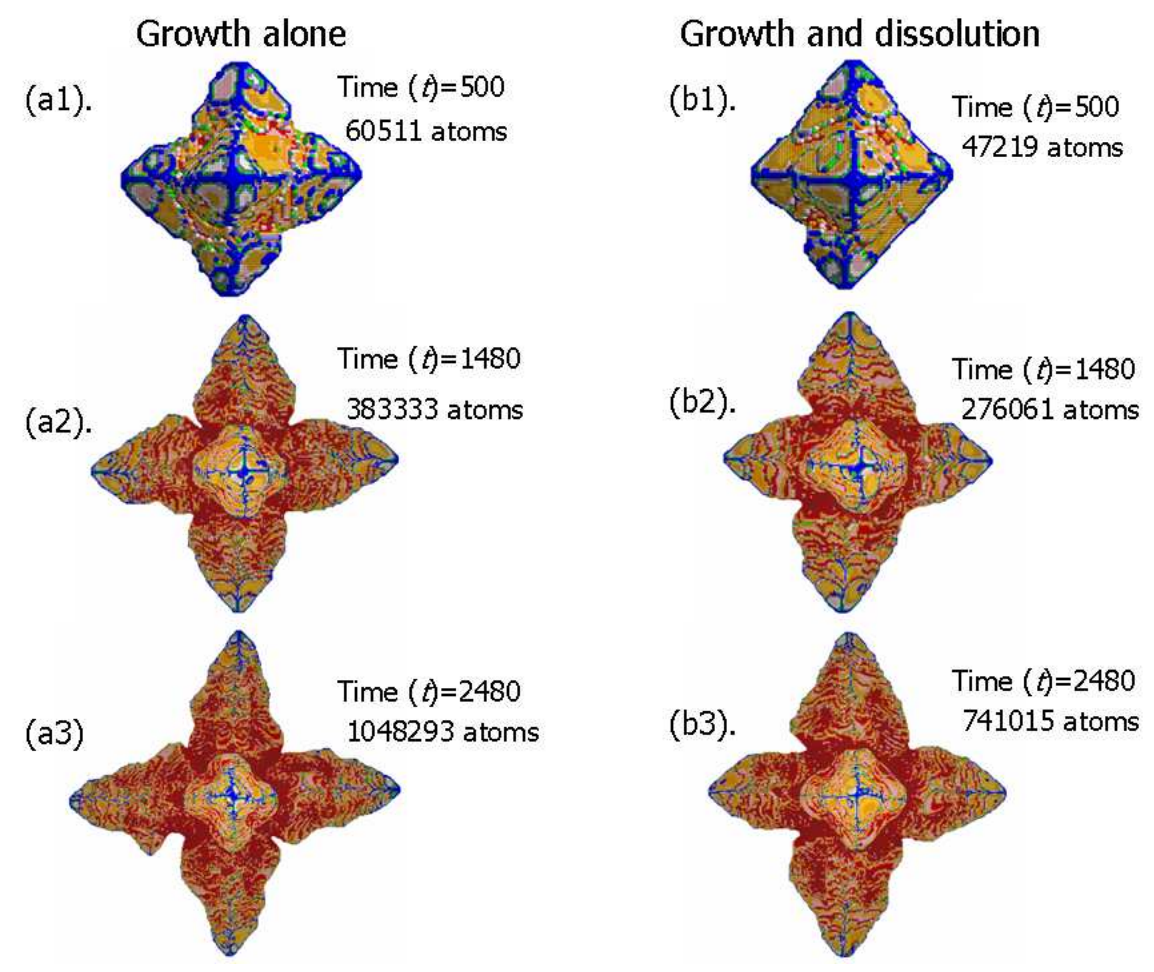

Figure 5.23: Six surface images from two separate simulations at three different times $(t=500,1480,2480)$ when $L e=1$ and $E_{N}=0.1 \mathrm{eV}$. Surface images ((a1), (a2) and (a3)) were taken from the growth alone simulation and correspond to the stage at which the crystal contains 60511 (a1), 383333 (a2) and 1048293 (a3) atoms. (b1), (b2) and (b3) images were taken from the growth and dissolution simulation, showing the crystal containing 47219 (b1), 276061 (b2) and 741015 (b3) atoms.

Figure 5.23 shows surface images from two separate simulations taken from three different times $(t=500,1480,2480)$. The surface images (a1), (a2), and (a3) were taken from the growth alone simulation and correspond to the stage at which the crystal contains 60511 (a1), 383333 (a2) 
and 1048293 (a3) atoms. (b1), (b2) and (b3) images were taken from the growth and dissolution simulation, showing the crystal containing 47219 (b1), 276061 (b2) and 741015 (b3) atoms. The number of atoms in growth alone simulation are higher at any particular time than in the growth and dissolution simulation. It can be seen from the surface image in the first column that more rough surfaces appear on the dendrite arms (edges and vertices) while the second column surface images show smoother surfaces. Figure 5.23 (a3) shows the holes in the groove between the arms is much deeper and the crystal has thinner arms compare to the image in (b3). The thicker arms in Figure 5.23 (b3) image illustrate that most of the dissolution atoms occur at rough surfaces especially the edges and vertices. Thus, the growth of the main stem of the arms is able to grow thicker due to the dissolution process being less active.

\subsection{Summary}

In this Chapter we have developed a model for calculating the nanocrystal growth into an undercooled binary melt. We used the front-tracking method, adopting an atomistic growth model in the spirit of the kinetic Monte Carlo simulations to track the free boundary. A numerical finite difference method was applied, using an explicit discretization to solve the continuum model for heat and diffusion equations at the solid-liquid interface. We provided a simple and straight forward approaches of developing a multiscale simulation method for the growth of nanocrystals in solution that couples a KMC description of the crystal relaxation process to solute reaction-diffusion equations. This model is easy to implement in the KMC code which allowed us to generate graphic representations in three dimensions. Very interesting results on the influence of the ratios of the rates of the different events on the occurrence of dendritic growth are presented and discussed. Also the results for growth from multiple seeds are impressive. Anisotropy is included in the model as a surface dif- 
fusion process and the growth rate of the dendrite was found to increase monotonically with increase in the surface anisotropy value. We also have developed a model that determines the shape of crystals as they grow and dissolve. The current Chapter also demonstrated quantitatively that crystals in the combined growth and dissolution situation obtain various results in terms of shape and growth rate compared to those in the growth alone. 


\section{Chapter 6}

\section{Diffusion and Growth KMC}

Here, we extend our KMC algorithms discussed in Chapters 4 and 5 to include the process of solute atom diffusion in a liquid environment. That is to allow a number of solute atoms to hop and to ensure that creation atoms are placed at the edge of the simulation cells so that they can diffuse inward. The solute atom hopping rate can be set at any rate, but preferably quite fast to mimic diffusion in the solvent. The diffusion processes can control the structure evolution of the growth surface and can be modelled using the KMC method. While the proposed technique can be applied to any surface system, our interest is in the examination of the behaviour as related to growth on nanocrystals structure when a solute atom diffusion event is included. A great advantage of the Diffusion and Growth KMC algorithm is that it allows for the possibility of nucleation and growth, whereas the continuum KMC algorithm only allows for growth. We will now turn our attention to using the Monte Carlo method to solve the diffusion equation in the next section. The KMC algorithm is discussed, including the creation of a single atom and its rate. Description of the surface diffusion is provided, and this is followed by the results and discussion. The introduction of dissolution events in the model is also discussed. 


\subsection{Diffusion equation using the Monte Carlo method}

To solve the diffusion equation using the Monte Carlo method [175], in some sense, is just an alternative method for solving the continuum equation in the previous Chapter. The diffusion equation in two dimensions is written as

$$
\frac{\partial C}{\partial t}=D\left(\frac{\partial^{2} C}{\partial x^{2}}+\frac{\partial^{2} C}{\partial y^{2}}\right)
$$

We replace the continuous derivatives of Equation (6.1) with their finite difference approximations, and choose the forward finite difference approximation as

$$
\frac{\partial C}{\partial t} \approx \frac{C_{i, j}^{n+1}-C_{i, j}^{n}}{t^{n+1}-t^{n}}=\frac{C_{i, j}^{n+1}-C_{i, j}^{n}}{\Delta t},
$$

where $C^{n}$ represents the solute concentration at the current time $t^{n}$ whereas $C^{n+1}$ represents the new solute concentration at $t^{n+1}$. The subscript $(i, j)$ refers to the location in the grid. The spatial derivatives of Equation (6.1) are replaced by a central finite difference approximation

$$
D\left(\frac{\partial^{2} C}{\partial x^{2}}+\frac{\partial^{2} C}{\partial y^{2}}\right) \approx D\left(\frac{C_{i+1, j}^{n}-2 C_{i, j}^{n}+C_{i-1, j}^{n}}{\Delta x^{2}}+\frac{C_{i, j+1}^{n}-2 C_{i, j}^{n}+C_{i, j-1}^{n}}{\Delta y^{2}}\right)
$$

Combining Equations (6.3) and (6.2) with Equation (6.1) gives

$$
C_{i, j}^{n+1}=C_{i, j}^{n}+\frac{D \Delta t}{\Delta h^{2}}\left(C_{i-1, j}^{n}+C_{i+1, j}^{n}+C_{i, j-1}^{n}+C_{i, j+1}^{n}-4 C_{i, j}^{n}\right),
$$

where $\Delta h=\Delta x=\Delta y$. The Monte Carlo algorithm uses the idea that the value of $C_{i, j}^{n+1}$ is an average over random walks of the boundary values.

In the current model, it was decided that the Monte Carlo method was too complex. Instead a simplified model was used in which newly cre- 
ated atom follow random walks from one empty lattice site to another before eventually nucleating. Random walks are related to the diffusion models and are a fundamental topic in discussions of Markov processes (described in Appendix B). More details of our approaches are described in the following sections.

\subsection{KMC algorithm}

The KMC algorithm used in the current Chapter assumes that single atom hops correspond to diffusion of solute atoms in the solution. The algorithm used here is based on the KMC algorithm developed in Chapter 4. However, the gas phase deposition process modelled in Chapter 4 is replaced by a model of diffusion in a solvent, much like that considered in Chapter 5, except that the diffusion equations are solved by explicit KMC solute simulations of solute atoms as discussed above. The current implementation of the first KMC algorithm involved minor changes to our deposition code by changing the process of deposition of a gas atom straight to the cluster to create a single atom at the cell edge. The Diffusion and Growth KMC model was initiated to enable us to conduct a comparative analysis of crystal morphology with the Continuum KMC Method in Chapter 5. There are two major types of events included in the current model: solute diffusion, modeled by single atom hopping, and surface diffusion. Other events are also included such as the dissolution process, which is considered for the simulation of the two different seed sizes.

\subsubsection{Creating a single atom at the cell edges}

The simulation is based on the situation where we have a spherical cluster of about 500 atoms placed in the middle of the simulation box. A single atom is created at the edge of the simulation cells and allowed to diffuse in by hopping randomly in towards a spherical cluster at a given temper- 
ature. The conditions for the placing of a single atom on the edge of the cell are that the lattice site must be unoccupied and has no neighbour in a fluid site. This condition is meant to minimise the chance of the single atoms at the edge joining together easily in the earlier stages. The rate of creating a single atom varies depending on the single atom hop, for example, if two or more single atoms join and stick together, then we have to decrease the creating rate to make sure there is no new nucleation formed apart from the main cluster. If there is no new nucleation formed then we have to increase the creating rate linearly. After placing a single atom on the edge of the cell, the atom starts to diffuse inward at a uniform rate. The single atom diffuses randomly from one empty lattice site to another empty lattice site and this is repeated accordingly until it comes into contact with atoms on the surface of the cluster. Note that the atoms on the surface of the crystal are allowed to hop at the same time as determined by their rates relative to solute diffusion in the KMC algorithm.

The hopping rate of a single atom in the fluid is set to be steady and greater than the surface diffusion rate. This is to enable the single atom to diffuse quite fast to mimic diffusion in the solute. It is assumed that each single atom hops independently and is randomly oriented into an empty lattice site. The rate of arrival of a single atom at the surface depends on the concentration of single atoms near the surface.

\subsubsection{Surface diffusion}

Along with the solute atoms hopping event, surface diffusion by all available surface atoms must be taken into account during a time interval. The diffusion rate of an atom is governed by the thermodynamic temperature $(T)$ of the system and the activation energy $E_{A}$ as discussed in Chapter 4. In this case, each jump to a neighbouring site will have a different probability determined by its activation energy, which in turn is assumed to depend upon the change in coordination number during the jump. We 
adopt the simple model in Ref. [116] with the energy barrier $E_{A}=i E_{0}$ of an atom proportional to its number $i$ of neighbours and to do so, we have employed again the energy $E_{0}=0.1 \mathrm{eV}$ throughout the simulation. The probability of a jump is given by Arrhenius law in Equation (4.1). The details of how to calculate the diffusion rate of an atom on the nanocrystal surface were briefly presented in the previous Section 4.1. Note we keep the temperature, $T=400 \mathrm{~K}$, constant in all the simulations.

\subsection{Results and Discussion}

We have studied the behaviour of nanocrystal structure when the diffusion event of a single atom originally placed at the edge of the simulation cell is included. Figure 6.1 shows the development of a dendrite and its sidebranches where the atoms that lie on the surface of the crystal are coloured according to coordination number $N_{i j k}$. These images correspond to four different times in a single simulation beginning from a spherical cluster of about 500 atoms. In the first image (a), the crystal contains about $4 \times 10^{3}$ atoms at $t=139$ and, in the second (b), there are about $3 \times 10^{4}$ atoms at $t=4946$. For this simulation a large value of the prefactor $v_{0} \approx 10^{13} \mathrm{~s}^{-1}$ was chosen, so that surface diffusion dominates, especially during the early stages of growth when the surface area is small. In this regime, the crystal grows close to its equilibrium Wulff shape, and the results are similar to that in Combe et al. and Schulze [116, 101]. If the crystal is seen to contain more facet regions and appears to grow very slowly, we increase the creating rate of a single atom (no neighbours) so that atoms are able to attach faster than the cluster can relax. The values of the flux that we actually used lie in the ranges of $\left(0.005 \times 10^{-4}-0.13 \times\right.$ $\left.10^{-4}\right)$ atoms $/ \mathrm{m}^{-2} \mathrm{~s}^{-1}$.

As discussed in our results in Chapter 5, the truncated octahedral shape can be understood as a competition between the two slowest growing facets, that is, the (100) and (111) facets. It can be seen that the nucleation is 
(a). Time $(t)=193$

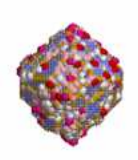

(c). Time $(t)=10415$

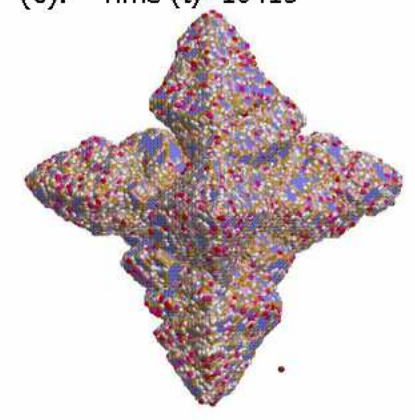

(b). Time $(t)=4946$
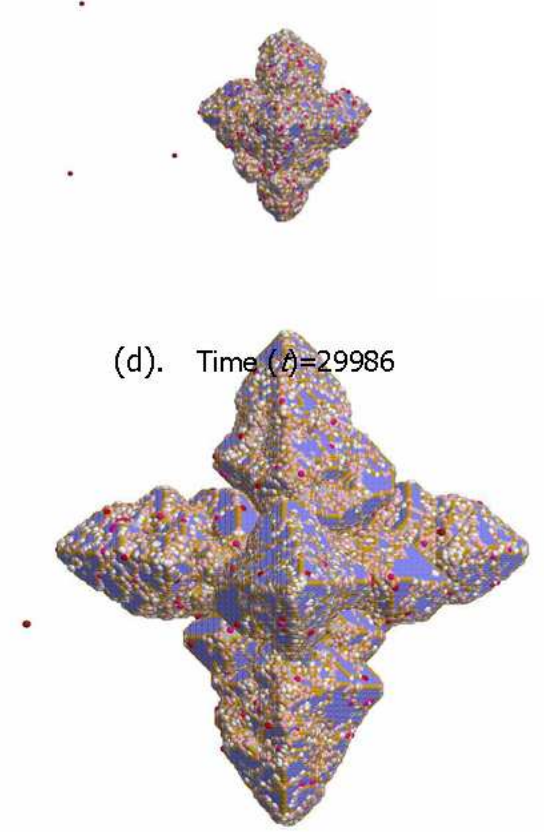

Figure 6.1: Four surface images of a single simulation at four different times. The top images (a) and (b) are taken from the early stages $(t=$ $139,4946)$ and correspond to the stage at which the crystal contains about $4 \times 10^{3}(\mathrm{a})$ and $3 \times 10^{4}(\mathrm{~b})$ atoms. The bottom images (c) and (d) are taken from the late stages $(t=10415,29986)$, showing the crystal containing about $2 \times 10^{5}(\mathrm{c})$ and $6.5 \times 10^{5}(\mathrm{~d})$ atoms. The images are coloured according to coordination number.

favoured on the (100) facet. Due to the geometry, more single atoms seem to attach to the (100) face compared to the (111) face. In addition, there is a net flux of surface atoms from a (111) face diffusing to a (100) facet, due to the availability of stable sites on the former. Figure 6.1 (c) and (d) indicate the surface images from the late stages $(t=10415,29986)$, showing the crystal containing about $2 \times 10^{5}$ (c) and $6.5 \times 10^{5}$ (d) atoms. The images show the surface instability leading to production of primary branches of a dendrite. 


\subsubsection{Multiple Seeds}

We have also extended the current model to the growth of multiple seeds. In the simulation presented, Figure 6.2 shows two identical seeds, spherical clusters of about 500 atoms, each with a different preferred growth. The results depict a simulation that was calculated in a $150 \times 150 \times 150$

(a). Time $(t)=173$

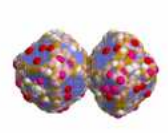

(c). Time $(t)=10415$

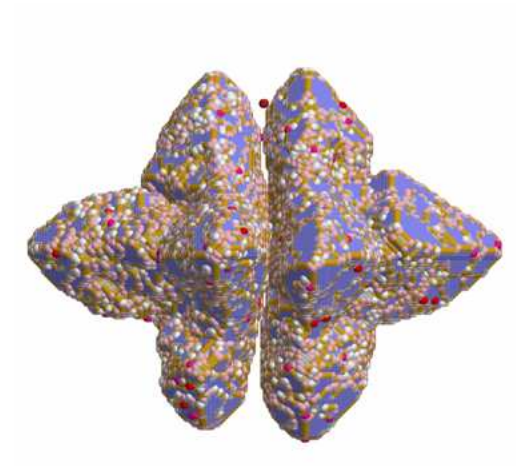

(b). Time $(t)=4645$

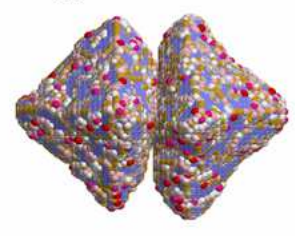

(d). Time $(t)=30025$

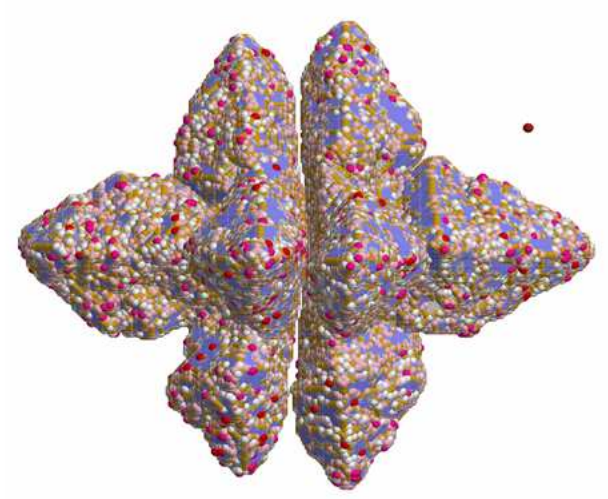

Figure 6.2: Evolution of two identical seeds of a single simulation at four different times. The top images (a) and (b) are taken from the early stages $t=173,4645$ and the bottom images (c) and (d) are taken from the late stages $t=17933,30022$. All images were coloured according to coordination number.

mesh in which the domain is kept at a fixed temperature $T=400 \mathrm{~K}$. The interfaces were allowed to merge when they came into contact. Figures 6.2 (a) and (b) show the early stages when the crystals started to merge. The last two images (c) and (d) correspond to much later stages of the simulation. There is less possibility of a single atom attaching between the 
surfaces of the two developing crystals because of a lack of open spaces (see Figure 6.3 (a) and (b)). Thus, these regions grow by surface diffusion rather than by solute attachment.

(a).

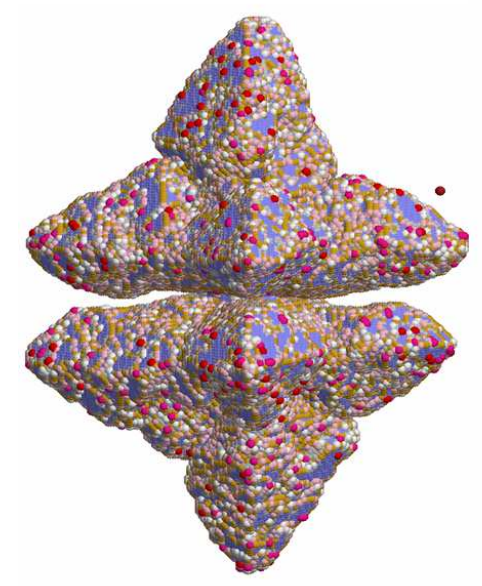

(b).

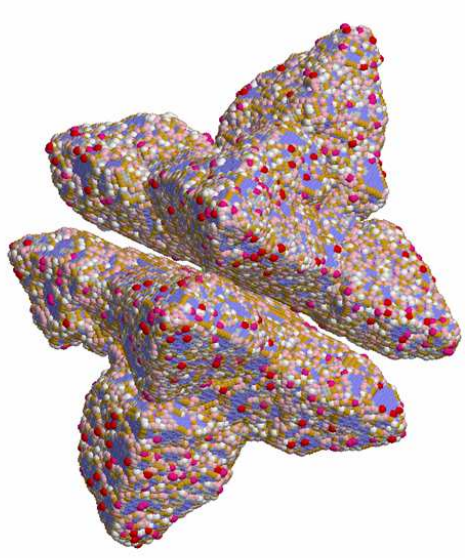

Figure 6.3: Two surface images from different view points of a single simulation at the same time. The images (a) and (b) are taken from the late stages coloured according to coordination number. These images show clearly the regions between the surfaces of the two crystals.

\subsubsection{Nature of crystal shape in two models}

Figure 6.4 shows four surface images of two separate simulations. Images (a1) and (a2) are taken from our Continuum-KMC Method described in Chapter 5 and images (b1) and (b2) from the Diffusion and Growth KMC method without considering the dissolution process. It can be seen that the two methods show the similar prediction of a dendrite shape. Figure 6.4 (a) and (b) show the solid atoms, coloured according to coordination, that lie on the surface of the crystal. In Figure 6.4 (a2), the solidifying atoms are coloured using the heat flux and in (b2) are coloured according to coordi- 
(a1).
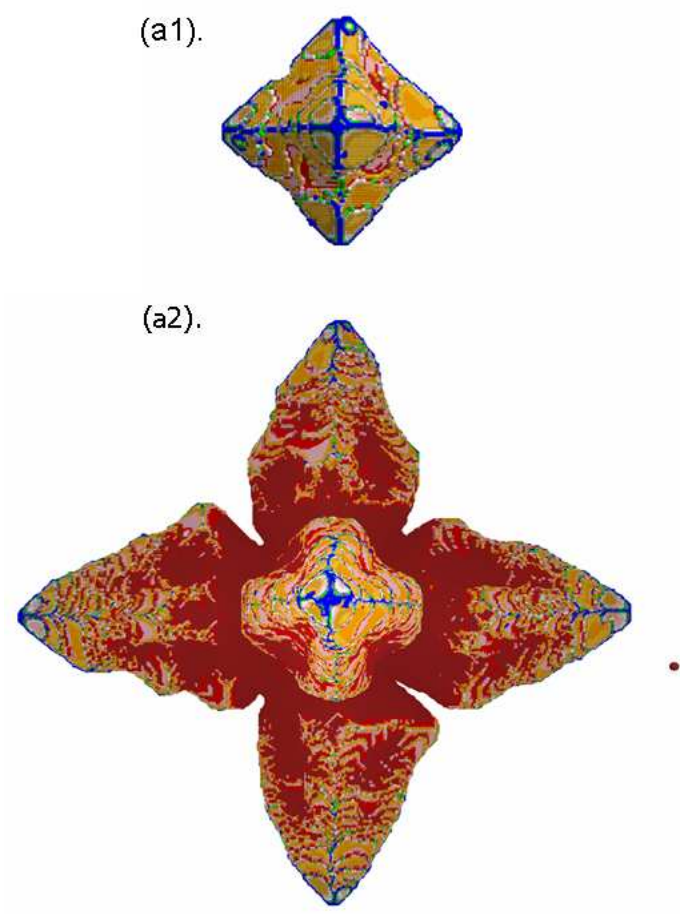

(b1).

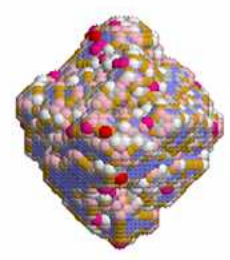

(b2).

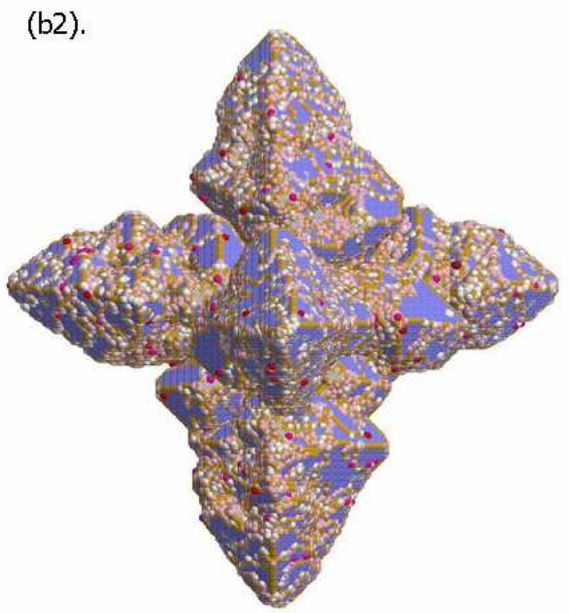

Figure 6.4: Images (a1) and (a2) are taken from our Continuum-KMC Method described in Chapter 5 and images (b1) and (b2) are taken from the Diffusion and Growth KMC method. Images (a1) and (b1) from the early stages and coloured according to coordination number. Images (a2) and (b2) from the late stages where (a) is coloured according to the temperature gradient while (b) is coloured according to coordination number.

nation number. The images (a2) and (b2) shown are from the late stages of two different simulations.

Note that we are not able to directly compare the two models for two main reasons:

1. The solute diffusion rate is set to be faster in the Diffusion and Growth KMC compared to the Continuum-KMC Method. This is to minimise the chance of new nucleation during the simulation, and

2. if there is a new nucleation apart from the main crystal then we have 
to disturb the simulation by decreasing the creating rate to avoid this.

\subsection{Dissolution}

In addition to the solute diffusion and surface diffusion processes, it is also important to include the dissolution event in the crystal growth. As discussed in Section 5.7, dissolution is a valuable mechanism for exposing crystal planes and generating crystal shapes that are not easily obtained through growth alone. Therefore we added the dissolution process in the current model in order to observe the morphology of crystal growth. In the next section the discussion of how we add the dissolution process to the current model is provided. This is followed by a comparative analysis of growth and dissolution. The dissolution and growth of two clusters is also presented.

\subsubsection{Dissolution Process}

The dissolution process is simulated using the KMC algorithm, similar to that used by Lasaga et al. [169], Lasaga et al. [170], and Meakin et al. [171]. Dissolution conditions are, the atom must be on the surface of the crystal and must have three or less than three nearest neighbours. If there is an atom which is able to dissolve back into the melt, then it must be dissolved to an unoccupied lattice position in the fluid (the dissolved atom must have at least one unoccupied site in the fluid site). A solid-on-solid model, which restricts the dissolution to the highest occupied solid site in each column of the lattice is also considered in our model. The solid-on solid approximation, which prevents overhangs from forming and simplifies the book keeping involved in the simulations, has been used extensively in KMC models for dissolution and growth [171, 30, 176]. In the KMC model, it is assumed that the rate of dissolution of an atom from the crystal 
surface into the fluid is given by

$$
R_{\text {diss }}= \begin{cases}v_{0} \exp \left[-n_{i} E_{a} / k_{B} T\right], & \text { if } n_{i} \leq 3 \\ 0, & \text { otherwise }\end{cases}
$$

where $E_{a}$ is the apparent dissolved activation energy. The activation energy that must be overcome to remove (dissolve) an atom is assumed to be proportional to the number of nearest neighbours (the coordination number) $n_{i}$. It is reasonable to use values of the activation energy for dissolution of typical minerals lying in the range of $30<E_{a}<75 \mathrm{~kJ} / \mathrm{mol}$ [177, $178,179]$.

\subsubsection{Comparing Growth and Dissolution}

When including dissolution we found that it is impossible to use the same creation rates from our growth simulation. The main reason for using different creation rates is that sometimes the dissolved atoms are in contact with the single atoms especially in the early stages of the simulation. We decrease the creation rates to minimize any new nucleation (note that rest of the parameters remain unchanged). Figure 6.5 shows surface images from two separate simulations taken at three different times $(t=921,16040,28165)$. The surface images (a1), (a2), and (a3) were taken from the growth alone simulation and (b1), (b2) and (b3) images were taken from the growth and dissolution simulation. Both simulations were started with a spherical cluster of about 500 atoms. It can be seen from the surface image in the first column that the surfaces appearing on the dendrite arms (edges and vertices) are rougher than when including the dissolution process (second column). The thicker arms in Figure 6.5 (b3) illustrate that most of the dissolution of atoms occur at rough surfaces especially the edges and vertices. 
(a1). Time $(t)=921$

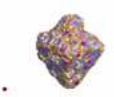

(a2). Time $(t)=16040$

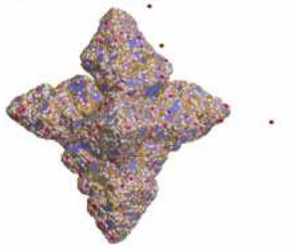

(a3). Time $(t)=28165$

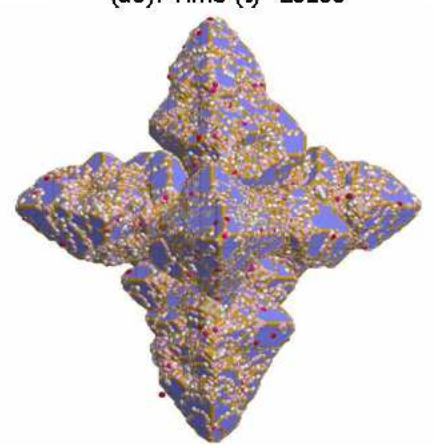

(b1). Time $(t)=921$

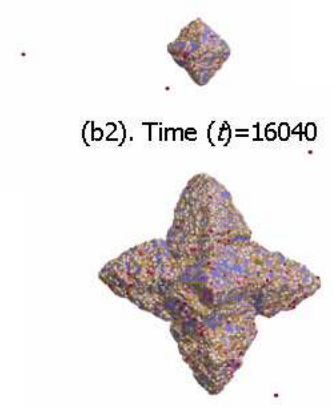

(b3). Time $(t)=28165$

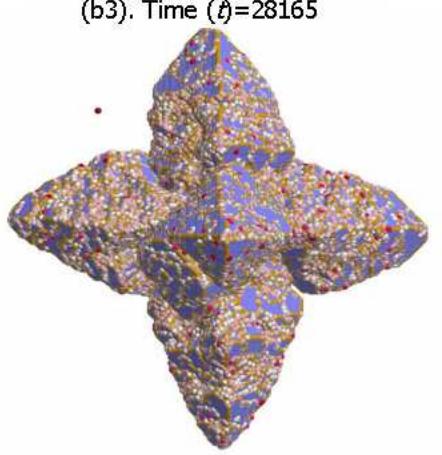

Figure 6.5: Surface images of two different simulations (growth alone simulation and growth and dissolution simulation) at three different times. The top images (a1) and (b1) are taken from the early stages at $t=921$. The middle images (a2) and (b2) are taken from the middle stages $\mathrm{T} t=16040$. The bottom images (a3) and (b3) are taken from the late stages at $t=28165$. The images were coloured according to coordination number.

\subsubsection{Ostwald ripening}

Ostwald ripening is a crystal growth process where atoms detach from a small crystal and attach to a larger crystal [180]. Small clusters disappear and large clusters grow through the Ostwald ripening mechanism due to the difference in chemical potential of different sized clusters [181]. The present work indicates that the cluster growth is completed through dissolution, single atom hop and surface diffusion mechanism when the separation among two different size clusters is not very far apart from each other. Figure 6.6 shows the six stages during the dissolute and growth of a 
(a). Time $(t)=7$

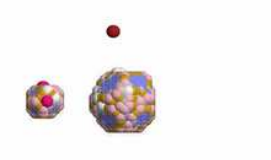

(d). Time $(t)=220$ (b). Time $(t)=83$

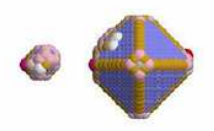

(e). Time $(t)=18415$ (c). Time $(t)=145$

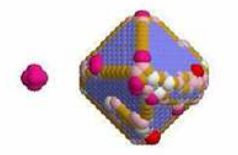

(f). Time $(t)=30560$
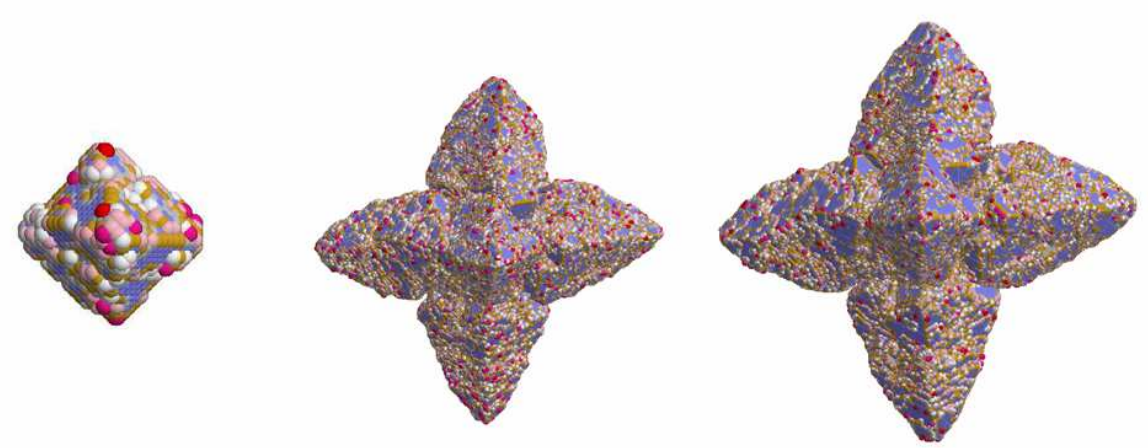

Figure 6.6: Evolution of two different cluster sizes of a single simulation at six different times. Images (a) and (b) show two surface images from the early stages with two different seeds that grow and dissolve at two different times $(t=7,83)$. Image (c) illustrates that the smaller seed is nearly dissolved and attached to the larger one at $t=145$. Image (d) indicates that the smaller seed is fully dissolved at $t=220$. The last two images (e) and (f) are taken from the late stages at $t=18415$ and 30560. All images were coloured according to coordination number.

pair of two different size clusters. The simulation is performed using two spherically symmetric clusters which originally contained 600 and 1500 atoms. It can be seen that the larger crystal grows at the expense of the smaller crystal as expected. This result is similar to most of the studies expecting that larger clusters melt or dissolve more slowly [182]. The larger cluster requires more time to melt or dissolve, permitting more relaxation to occur before reaching the smaller sizes. Figures 6.6 (a) and (b) depict two surface images from the early stages, showing two different seeds that grow and dissolve at two different times $(t=7,83)$. Image (c) illustrates 
that the small seed is nearly dissolved and attached to the larger one at $t=145$. Image (d) indicates that smaller seed is fully dissolved at $t=220$. The last two images (e) and (f) are taken from the late stages at two different time $(t=18415,30560)$.

\subsubsection{Two clusters of the same sizes dissolve and grow}

(a). Time $(t)=0$
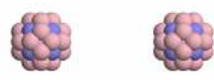

(d). Time $(t)=146$

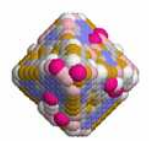

(b). Time $(t)=50$

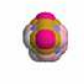

(e). Time $(t)=155$

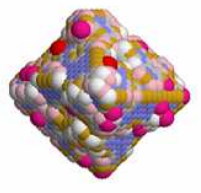

(c). Time $(t)=100$
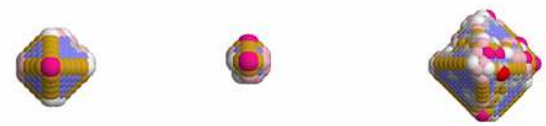

(f). Time $(t)=195$

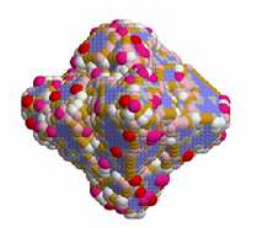

Figure 6.7: Evolution of two clusters initially of same size of a single simulation at six different times. Image (a) shows the initial configuration of the two clusters. Images (b), (c) and (d) show the clusters growing and dissolving at three different times $(t=50,100,146)$. Image (e) indicates that one of the clusters is fully dissolved at $t=155$. In the last image (f) arms of the dendrite have started to grow. All images were coloured according to coordination number.

The model extends the growth of two clusters of the same size, which is completed through dissolution, single atom hop and surface diffusion mechanisms. The simulation is performed using two spherically symmet- 
ric clusters which originally contained 87 atoms. The initial configuration of the two clusters is shown in Figure 6.7 (a). Figure 6.7 (b), (c), and (d) show that one of the clusters is dissolved and attached to the other cluster. The last two images (e) and (f) illustrate that one of the clusters is completely dissolved. Then the remaining crystal will grow and obtain a similar shape as in Figure 6.6 (f).

\subsection{Summary}

We have constructed a KMC model to simulate the process of a single atom diffusion event in a liquid environment. That is to allow a single atom to hop and to ensure that creating atoms are placed at the edge of the simulation cells so that they can diffuse inward. The single atom hopping rate can be set at any rate, but preferably quite fast to mimic diffusion in the solute. The dendrite shape of the crystal in the current model is similar to our result in Chapter 5. The current model shows the ability to model multiple seeds. We also discussed what happens if the dissolution event is included in the current model. We demonstrated that crystals have a similar crystal shape as we discussed in Chapter 5 . The dissolution process in two different size clusters shows that the atoms from the smaller cluster dissolve and appear to join the larger cluster. 


\section{Chapter 7}

\section{Conclusion and Future Work}

\subsection{Conclusion}

The current study explored this main goal: To develop a multiscale simulation method for the growth of nanocrystals in solution that couples a KMC description of the crystal relaxation process to solute reaction diffusion equations. This goal set out to collect in-depth information for a better understanding of the phase crystal growth using the KMC method, supported by a quantitative analysis of the results. Therefore, it was necessary to couple the theory of crystal relaxation with the mathematical understanding of solute diffusion fields.

The kinetic Monte Carlo model was developed to perform realistic simulations over a useful range of growth and dissolution conditions. The emphasis of this work was to establish a model which can serve as a development platform for other more advanced models. The main motivation behind this work was to advance the current state of knowledge and understanding of crystal growth mechanisms. The thesis was divided into three parts: the Deposition and Growth KMC method, the ContinuumKMC Method, and the Diffusion and Growth KMC method. These phenomena were discussed and analyzed by adopting a discrete, atomistic model in the spirit of the KMC simulation which is popular in the growth 
and dissolution literature. This led to interesting results and valuable insights. We will now summarize the findings, and review the main goals of the research, and what we have achieved in terms of the research objectives.

In Chapter 4 the interplay of the deposition and surface diffusion rates were demonstrated in the gas phase. The first case, when deposition rate is far greater than hopping rate, indicated that the cluster grows very fast. As the simulation time increased, the shape of the crystal became completely spherical. The physical picture of the crystallite above the roughening temperatures has many visible kinks and steps, indicating that the continuous approximation for the curvature might be valid; the mass transfer of solute is via atomic diffusion from kinks or steps from the high curvature regions to the existing kinks or steps of the low curvature regions. Below this temperature, however, large facets do appear in the low curvature regions and no kinks or steps are available, thus preventing the diffusing atoms from sticking there. The final case showed the growth of the cluster is not as fast as in the first case, but leads to the same structure. The solution in Chapter 4 has contributed to guide us to be able to respond to the main goal of the research.

The most essential underlying finding in the current study is that the KMC appears to be a promising model for the simulation of dendrite growth on atomistic scales. We adopted an atomistic growth model that uses a KMC technique to track the free boundary. The model allows for both phase change and exchange between liquid and solid atoms on the surface of the crystal and is coupled to a continuum model for heat and diffusion equations at the solid-liquid interface. The present study has demonstrated that the KMC algorithm is useful in contributing to our understanding of solution phase crystal growth, especially of nanocrystal growth. For small length and time scales, this approach provides a simple, effective front tracking with fully resolved atomistic detail. The technique was used to make realistic predictions regarding surface morphology of 
crystals.

We have achieved all the research objectives mentioned in Chapter 1. Firstly we showed, that the KMC algorithm is suitable to study the process of solidification in order to explore the evolution and morphology of crystal structure. Secondly, the model was then applied to the numerical finite difference method by using an explicit discretization to solve the heat and diffusion equations. The solute concentration $\left(C_{L}\right)$ on the liquid site of the interface was calculated. Finally, the solidification and hopping rates were calculated. The anisotropy is included in the model as a surface diffusion process, and the growth rate of a dendrite is found to increase monotonically with the surface anisotropy. Thus, the branching process (including secondary and nascent tertiary branches) occurs at earlier stages of growth when the value of the surface anisotropy is increased. On the contrary, at low surface anisotropy the crystallite is fully faceted.

In Chapter 5, as expected from the large value of Lewis number, the thickness of the thermal boundary layer is much larger than that of the solutal boundary layer. Even though the Lewis number is large, the initial melt concentration is made low enough that the interface temperature is significantly different from the far-field value. The tip velocity when Lewis number $L e=1$ of the Continuum-KMC model is successfully compared with the prediction by Schulze [101] and Tan et al. [139]. The prediction of tip velocity by Karma [162] also agree well with our result when the $L e=1000$.

The kinetic Monte Carlo algorithm for the deposition code in Chapter 4 was extended to include the process of a solute diffusion in the fluid in Chapter 6. We placed a series of single atoms at the edge of the simulation cells and allowed them to diffuse inward. The uniform rate of a single atom was set quite fast to imitate diffusion in the solute. The final shape of the crystal in the two models, the Continuum-KMC model and the Diffusion and Growth KMC model, is similar.

Although the numerical results show very complicated interface mor- 
phologies, topological changes can be simulated properly and efficiently. The thesis may not completely solve the outstanding problem of selective high quality crystal growth but it does provide the metrology by which selective growth may be investigated further. This study has a lot of predictive capability however several issues have to be addressed before it will be able to perform industrially relevant simulations.

\subsection{Recommendations for future work}

The model itself can be refined by considering the following:

- There is every reason to expect that the range of parameters addressable by this technique can be greatly increased by coupling it to more efficient methods for solving the heat and diffusion equations.

- The high fluctuation of the mass difference is due to the influence of the random number and average velocity during the simulation. This can be modified so that mass is strictly conserved by applying the growth model to a discrete model for mass transfer.

- There is a need for the current model to simulate a specific element like gold, silver or other metals by taking realistic parameters and comparing the results to experimental data.

- This method will enable researchers to explain why certain crystal shapes form under particular conditions during growth, and may enable nanotechnologists to design techniques for growing nanocrystals with specific shapes for a variety of applications, from catalysis to the medicine field and electronics industry. 


\section{Appendix A}

\section{Stochastic processes}

A stochastic process is the time evolution of a stochastic variable, $Y$. Thus the stochastic process is described by its position $Y(t)$ at time $t \in[0,1], t \in$ $[0, \infty]$, or $t \in[0,1, \ldots, n]$. A stochastic variable is defined by specifying:

1. the set of possible values (called "range", "set of states", "sample state" or "phase space"), and

2. the probability distribution over this set. The set can be discrete (e.g. number of molecules of a component in a reacting mixture), continuous (e.g. the velocity of a Brownian particle) or multidimensional. In the latter case, the stochastic variable is a vector (e.g. the three velocity components of a Brownian particle).

Example for stochastic processes are Brownian motion, random walks, Poisson and Markov processes. Figure A.1 gives a more intuitive interpretation of a stochastic process. At successive times, the most probable value of $Y$ have been drawn as heavy dots. The most probable trajectory can be selected from such a picture. Two or more trajectory can occur with equal probability. 


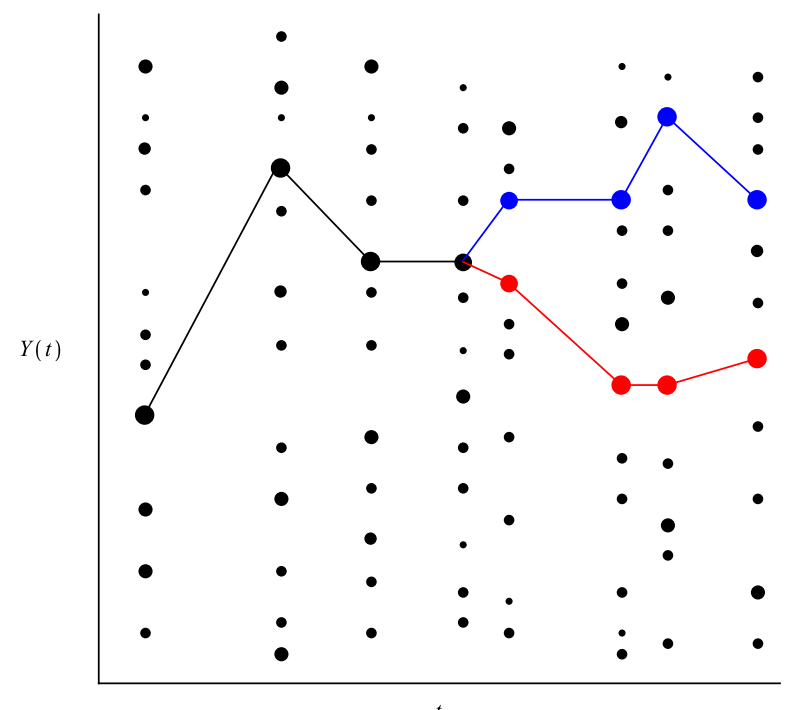

Figure A.1: A schematic interpretation of a stochastic process, $Y$, as a function of time $t$. The heavy dots indicates the most probable values of $Y$. 


\section{Appendix B}

\section{Markov Processes}

In order to understand the Markov processes, the conditional probability should be defined.

\section{B.1 The Conditional Probability}

The conditional probability $P_{1 \mid 1}\left(y_{2}, t_{2} \mid y_{1}, t_{1}\right)$ is defined through the following relation:

$$
P_{2}\left(y_{1}, t_{1} ; y_{2}, t_{2}\right)=P_{1 \mid 1}\left(y_{2}, t_{2} \mid y_{1}, t_{1}\right) P_{1}\left(y_{1}, t_{1}\right)
$$

which means that the joint probability $P_{2}$ of finding the system in configuration $y_{1}$ at $t_{1}$ and $y_{2}$ at $t_{2}$ is equaled to the probability of finding $y_{2}$ at $t_{2}$, given $y_{1}$ at $t_{1}$ multiplied by the probability $P_{1}\left(y_{1}, t_{1}\right)$ of finding $y_{1}$ at $t_{1}$. In general, the conditional probability is given as

$$
\begin{array}{r}
P_{1 \mid n}\left(y_{n+1}, t_{n+1} ; \ldots ; y_{n+l}, t_{n+l} \mid y_{1}, t_{1} ; \ldots ; y_{n}, t_{n}\right)= \\
\frac{P_{n+1}\left(y_{1}, t_{1} ; \ldots ; y_{n}, t_{n} ; y_{n+1}, t_{n+1} ; \ldots ; y_{n+l}, t_{n+l}\right)}{P_{n}\left(y_{1}, t_{1} ; \ldots ; y_{n}, t_{n}\right)}
\end{array}
$$

where $P_{n}\left(y_{1}, t_{1} ; \ldots ; y_{n}, t_{n}\right)$ is the probability that the stochastic variable, $Y$, assumes the value $y_{1}$ at $t_{1}, y_{2}$ at $t_{2}$ and up to $y_{n}$ at $t_{n}$. 


\section{B.2 Markov Property}

The Markov process is defined by the following relation, which is called the Markov property, for any set of $n$ successive times (i.e., $t_{1}<t_{2}<\ldots<$ $\left.t_{n}\right)$ [183]:

$$
P_{1 \mid n-1}\left(y_{n}, t_{n} \mid y_{n-1}, t_{n-1} ; \ldots ; y_{1}, t_{1}\right)=P_{1 \mid 1}\left(y_{n}, t_{n} \mid y_{n-1}, t_{n-1}\right) .
$$

The Markov property merely expresses that, for a Markov process, the probability of a transition from a value $y_{n-1}$ at time $t_{n-1}$ to a value $y_{n}$ at time $t_{n}$, depends only the value of $y$ at the time $t_{n-1}$, and not on the previous history of the system. A Markov process is only dependent on $P_{1}\left(y_{1}, t_{1}\right)$ and $P_{1 \mid 1}\left(y_{2}, t_{2} \mid y_{1}, t_{1}\right)$ and subsequently the whole hierarchy can be reconstructed from them, where $P_{1 \mid 1}$ is called the transition probability. For example, if $t_{1}<t_{2}<t_{3}$, it can be written as

$$
\begin{array}{r}
P_{3}\left(y_{1}, t_{1} ; y_{2}, t_{2} ; y_{3}, t_{3}\right)=P_{2}\left(y_{1}, t_{1} ; y_{2}, t_{2}\right) P_{1 \mid 2}\left(y_{3}, t_{3} \mid y_{1}, t_{1} ; y_{2}, t_{2}\right) \\
=P_{1}\left(y_{1}, t_{1}\right) P_{1 \mid 1}\left(y_{2}, t_{2} \mid y_{1}, t_{1}\right) P_{1 \mid 1}\left(y_{3}, t_{3} \mid y_{2}, t_{2}\right)
\end{array}
$$

The above equation can be continued successively to find all the probabilities $P_{n}$. The Markov property states that, to make predictions of the behaviour of a system in the future, it suffices to consider only the present state of the system and not the past history. The details of the Markov processes can be found in $[184,185,186]$. 


\section{Appendix C}

\section{Derivation of the Master Equation}

The derivation of the Master Equation presented here is significantly simplified and a more complete derivation can be found in [76, 86, 185]. In order to obtain such a Master Equation, one has to first introduce the conditional probability described in B.1. The derivation starts by integrating Equation (B.4) with respect to $y_{2}$. Thus, for $t_{1}<t_{2}<t_{3}$ it follows that

$$
\begin{aligned}
\int P_{3}\left(y_{1}, t_{1} ; y_{2}, t_{2} ; y_{3}, t_{3}\right) d y_{2} & =\int P_{1}\left(y_{1}, t_{1}\right) P_{1 \mid 1}\left(y_{2}, t_{2} \mid y_{1}, t_{1}\right) P_{1 \mid 1}\left(y_{3}, t_{3} \mid y_{2}, t_{2}\right) d y_{2} \\
P_{2}\left(y_{1}, t_{1} ; y_{3}, t_{3}\right) & =P_{1}\left(y_{1}, t_{1}\right) \int P_{1 \mid 1}\left(y_{2}, t_{2} \mid y_{1}, t_{1}\right) P_{1 \mid 1}\left(y_{3}, t_{3} \mid y_{2}, t_{2}\right) d y_{2} \\
P_{1 \mid 1}\left(y_{3}, t_{3} \mid y_{1}, t_{1}\right) P_{1}\left(y_{1}, t_{1}\right) & =P_{1}\left(y_{1}, t_{1}\right) \int P_{1 \mid 1}\left(y_{2}, t_{2} \mid y_{1}, t_{1}\right) P_{1 \mid 1}\left(y_{3}, t_{3} \mid y_{2}, t_{2}\right) d y_{2} .
\end{aligned}
$$

Dividing both sides of Equation C.1 by $P_{1}\left(y_{1}, t_{1}\right)$ gives the conditional probability of Markov processes obeying the Chapman-Kolmogorov equation

$$
P_{1 \mid 1}\left(y_{3}, t_{3} \mid y_{1}, t_{1}\right)=\int P_{1 \mid 1}\left(y_{3}, t_{3} \mid y_{2}, t_{2}\right) P_{1 \mid 1}\left(y_{2}, t_{2} \mid y_{1}, t_{1}\right) d y_{2} .
$$

This so-called Chapman-Kolmogorov equation states that a process starting at $t_{1}$, with value $y_{1}$, reaches $y_{3}$ at $t_{3}$ via any one of the possible values $y_{2}$ 
at the intermediate time $t_{2}$. If the Markov process is stationary, the transition probability $P_{1 \mid 1}$ depends only on the time interval $\tau$

$$
P_{1 \mid 1}\left(y_{2}, t_{2} \mid y_{1}, t_{1}\right)=T_{\tau}\left(y_{2} \mid y_{1}\right), \quad \text { where } \tau=t_{2}-t_{1} .
$$

Here $T_{\tau}$ denotes the transitional probability within the time interval $\tau$ from a state $y_{1}$ to a state $y_{2}$. Using the Chapman-Kolmogorov equation for $T_{\tau}$ gives

$$
T_{\tau+\tau_{0}}\left(y_{3} \mid y_{1}\right)=\int T_{\tau_{0}}\left(y_{3} \mid y_{2}\right) T_{\tau}\left(y_{2} \mid y_{1}\right) d y_{2} .
$$

The Master Equation can consequently be derived from the ChapmanKolmogorov equation. Taking the transition probability $T_{\tau_{0}}$ and expand it in a Taylor series over zero, considering small $\tau_{0}$, then the transition probability becomes

$$
T_{\tau_{0}}\left(y_{3} \mid y_{2}\right)=\delta\left(y_{2}-y_{3}\right)+\tau_{0} W\left(y_{3} \mid y_{2}\right)+O\left(\tau_{0}^{2}\right) .
$$

This equation defines $W\left(y_{3} \mid y_{2}\right)$ as the transition rate (transition probability per unit time) to go from $y_{2}$ to $y_{3}$. The delta function expresses that the probability to stay at the same state after time zero is one, whereas the time to change after time zero equals zero. Equation C.3 must satisfy the normalization property $\int T_{\tau_{0}}\left(y_{3} \mid y_{2}\right) d y_{2}=1$. Therefore the integral over $y_{3}$ must equal one. In order for that to happen, the above form must be corrected in the following sense:

$$
T_{\tau_{0}}\left(y_{3} \mid y_{2}\right)=\left(1-W_{t o t}\left(y_{2}\right) \tau_{0}\right) \delta\left(y_{3}-y_{2}\right)+\tau_{0} W\left(y_{3} \mid y_{2}\right)+O\left(\tau_{0}^{2}\right) .
$$

In the first term, the factor $\left(1-W_{\text {tot }}\left(y_{2}\right) \tau_{0}\right)$ gives the probability to remain in state $y_{2}$ up to time $\tau_{0}$. That means that $W_{\text {tot }}\left(y_{2}\right)$ is the total probability to 
leave the state $y_{2}$, defined as

$$
W_{t o t}\left(y_{2}\right)=\int W\left(y_{3} \mid y_{2}\right) d y_{3}
$$

Inserting this into the Chapman-Kolmogorov equation results in

$$
T_{\tau+\tau_{0}}\left(y_{3} \mid y_{1}\right)=\left(1-W_{t o t}\left(y_{3}\right) \tau_{0}\right) T_{\tau}\left(y_{3} \mid y_{1}\right)+\tau_{0} \int W\left(y_{3} \mid y_{1}\right) T_{\tau}\left(y_{2} \mid y_{1}\right) d y_{2},
$$

and dividing it by $\tau_{0}$ gives

$\frac{T_{\tau+\tau_{0}}\left(y_{3} \mid y_{1}\right)-T_{\tau}\left(y_{3} \mid y_{1}\right)}{\tau_{0}}=\int W\left(y_{3} \mid y_{2}\right) T_{\tau}\left(y_{2} \mid y_{1}\right) d y_{2}-\int W\left(y_{2} \mid y_{3}\right) T_{\tau}\left(y_{3} \mid y_{1}\right) d y_{2}$,

in which we have use the definition of $W_{\text {tot }}$. In the limit $\tau_{0} \rightarrow 0$ yields the differential version of the Chapman-Kolmogorov equation:

$$
\frac{\partial}{\partial \tau} T_{\tau}\left(y_{3} \mid y_{1}\right)=\int W\left(y_{3} \mid y_{2}\right) T_{\tau}\left(y_{2} \mid y_{1}\right) d y_{2}-\int W\left(y_{2} \mid y_{3}\right) T_{\tau}\left(y_{3} \mid y_{1}\right) d y_{2},
$$

This equation is valid for the transition probability of a stationary Markov process obeying equation is called the Master Equation. Rewriting Equation (C.7) and suppressing redundant indices gives the

$$
\frac{\partial P(y, t)}{\partial t}=\int W\left(y \mid y^{\prime}\right) P\left(y^{\prime}, t\right) d y^{\prime}-\int W\left(y^{\prime} \mid y\right) P(y, t) d y^{\prime} .
$$

If the range of $Y$ is a discrete set of states with labels $C$, the equation reduces to

$$
\frac{\partial p_{n}(t)}{\partial t}=-\sum_{n^{\prime}} W_{n^{\prime} n} p_{n}(t)+\sum_{n^{\prime}} W_{n n^{\prime}} p_{n^{\prime}}(t)
$$

which is the Master Equation (3.1) given in Subsection 3.3.1. 


\section{Appendix D}

\section{Proof of Equation (3.9)}

If $N$ is a discrete random variable taking values in the non-negative integers $\{0,1, \ldots\}$, then the probability-generating function $G(s, t)$ of $N$ is defined as

$$
G(s, t)=\sum_{n=0}^{\infty} s^{n} p_{n}(t),
$$

where $p$ is the probability mass function of $N$ and $s$ is independent of time $t$. Note that the subscripted notation $p_{n}$ is often used to emphasize that this pertains to a particular random variable $N$, and to its distribution. Differentiating Equation (D.1) and substituting Equation (3.1) into it gives:

$$
\begin{aligned}
\frac{d G(s, t)}{d t} & =\sum_{n=0}^{\infty} s^{n} \dot{p}_{n}(t), \quad \text { where } \dot{p}_{n}(t) \text { is given in Equation (3.8) } \\
& =\sum_{n=0}^{\infty} s^{n}\left[q\left(p_{n-1}-p_{n}\right)\right] \\
& =q s \sum_{n=1}^{\infty} s^{n-1} p_{n-1}(t)-q \sum_{n=0}^{\infty} s^{n} p_{n}(t) \\
d G(s, t) & =q(s-1) G(s, t) d t \\
G(s, t) & =G(s, 0) e^{q(s-1) t}
\end{aligned}
$$


Note, that $G(s, 0)=\sum_{n=0}^{\infty} s^{n} p_{n}(0)=1$ and only the $n=0$ term survives due to the initial condition $p_{n}(0)=\delta_{n, 0}$ given in Equation (3.7). Thus,

$$
\begin{aligned}
G(s, t) & =e^{q(s-1) t} \\
& =e^{q s t} e^{-q t} \\
& =\sum_{n=0}^{\infty} \frac{(q s t)^{n}}{n !} e^{-q t} \\
& =\sum_{n=0}^{\infty} s^{n} p_{n}(t) \\
p_{n}(t) & =\frac{(q t)^{n}}{n !} e^{-q t},
\end{aligned}
$$

which is Equation (3.9) given in Subsection 3.3.2. 


\section{Appendix E}

\section{Transition State Theory (TST)}

Transition state theory (TST) is a method for calculating the rate of occurrence of rare events. In the theory developed by Eyring [187], during a chemical reaction the initial reactants, which are in a stable state with low potential energy, interact to form an activated complex (a transition state). This activated complex has high potential energy and is unstable. The activated complex rapidly decays to the stabler (lower potential energy) products. Figure E.1 outlines the TST in which a two state problem is presented, consisting of the initial state $i$ (reactant), the final state $j$ (product) and the activated complex (transition state) $x_{0}$ separating the two states. By assuming a canonical ensemble, it is possible to derive an expression for the rate at which the infinite heat bath pushes the atom at state $i$ through the activated complex to state $j$. Let us assume that an atom is at the position, $\Delta x$, around the activated complex and it is moving towards state $j$. The probability of finding the atom in this region around the activated complex $x_{0}$ is given by [188]

$$
P(\Delta x)=\frac{\exp \left(-\beta V\left(x_{0}\right)\right)}{\int_{-\infty}^{x_{0}} \exp (-\beta V(x)) d x},
$$

where $\beta=\left(k_{B} T\right)^{-1}, k_{B}$ is Boltzmann's constant, $T$ is the temperature of the system, and $V(x)$ is the potential energy at state $x$. The upper limit of 


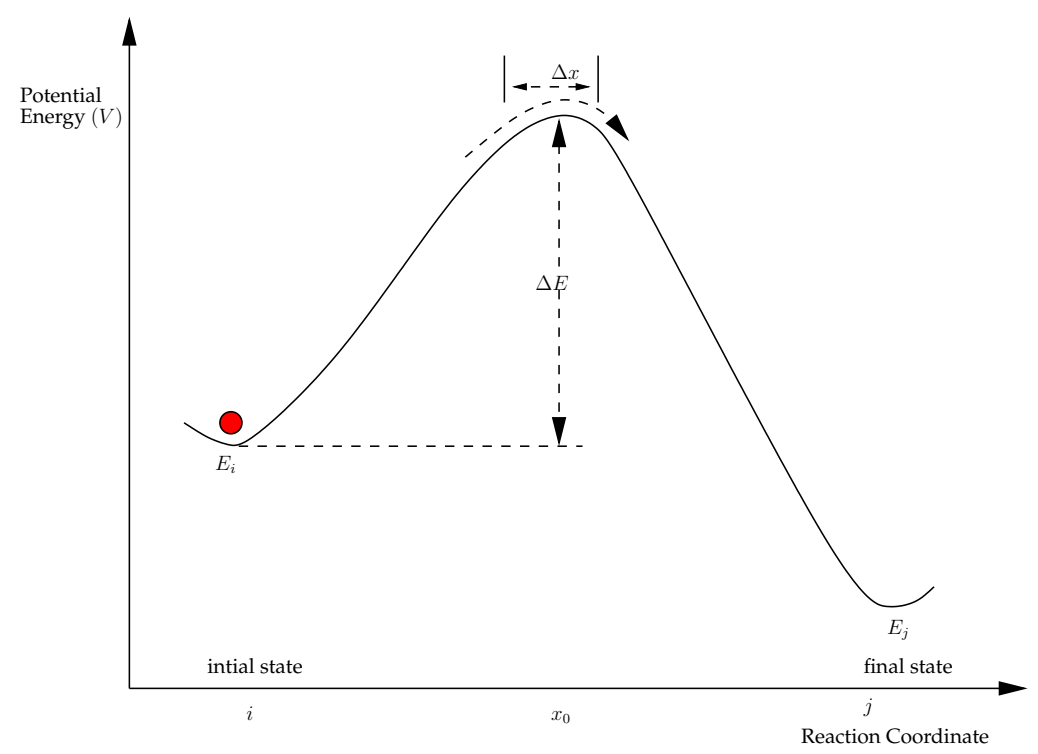

Figure E.1: Schematic illustration of the lowest free energy path for a thermally activated jump of an adatom from state $i$ to state $j$ over the saddle point $x_{0}$.

the normalization integral in Equation (E.1) indicates the assumption that the atom resides at the initial state $i$. Thus, the probability density for the atom having velocity $v$ is written as:

$$
\begin{aligned}
P(v) & =\frac{\exp \left(-\frac{1}{2} \beta m v^{2}\right)}{\int_{-\infty}^{\infty} \exp \left(-\frac{1}{2} \beta m v^{2}\right) d v}, \text { where } V\left(x_{0}\right)=\frac{1}{2} m v^{2}, \\
& =\left(\frac{m \beta}{2 \pi}\right)^{\frac{1}{2}} \exp \left(-\frac{1}{2} \beta m v^{2}\right) .
\end{aligned}
$$

The atom with positive velocity $v$ will enter the final state $j$ in a short time interval $\Delta t$, providing that $\Delta x$ is smaller than $v \Delta t$. Then the total probability that the atom will jump from site $i$ to site $j$ is

$$
P_{i \rightarrow j}=\int_{0}^{\infty} P(v) P(\Delta x=v \Delta t) d v .
$$


Equation (E.3) may then be rewritten incorporating Equation (E.2), which gives the expression for the transition rate

$$
v_{i \rightarrow j}=\frac{\exp \left(-\beta V\left(x_{0}\right)\right)}{(2 \pi m \beta)^{\frac{1}{2}} \int_{-\infty}^{x_{0}} \exp (-\beta V(x)) d x} .
$$

It is thus clear that the problem reduces to solve the integral in Equation (E.4). We shall always assume the condition $V\left(x_{0}\right)>>k_{B} T$ then $V(x)$ can be replaced with its expansion to the second order. The rate of a process can therefore be expressed as:

$$
v_{i \rightarrow j}=v_{i} \exp \left(-\Delta E / k_{B} T\right)
$$

where $v_{i}=\frac{k_{B} T}{h}$ is the prefactor, $h$ is Planck's constant, and $\Delta E$ is the energy barrier. TST is described in more detail in references [189, 190]. 


\section{Appendix F}

\section{Supplementary Code}

In this appendix, extracts from the programs written in this study are given. The program was written in Fortran.

PART 1: Subroutine for clearing an atom and updating the lattice site.

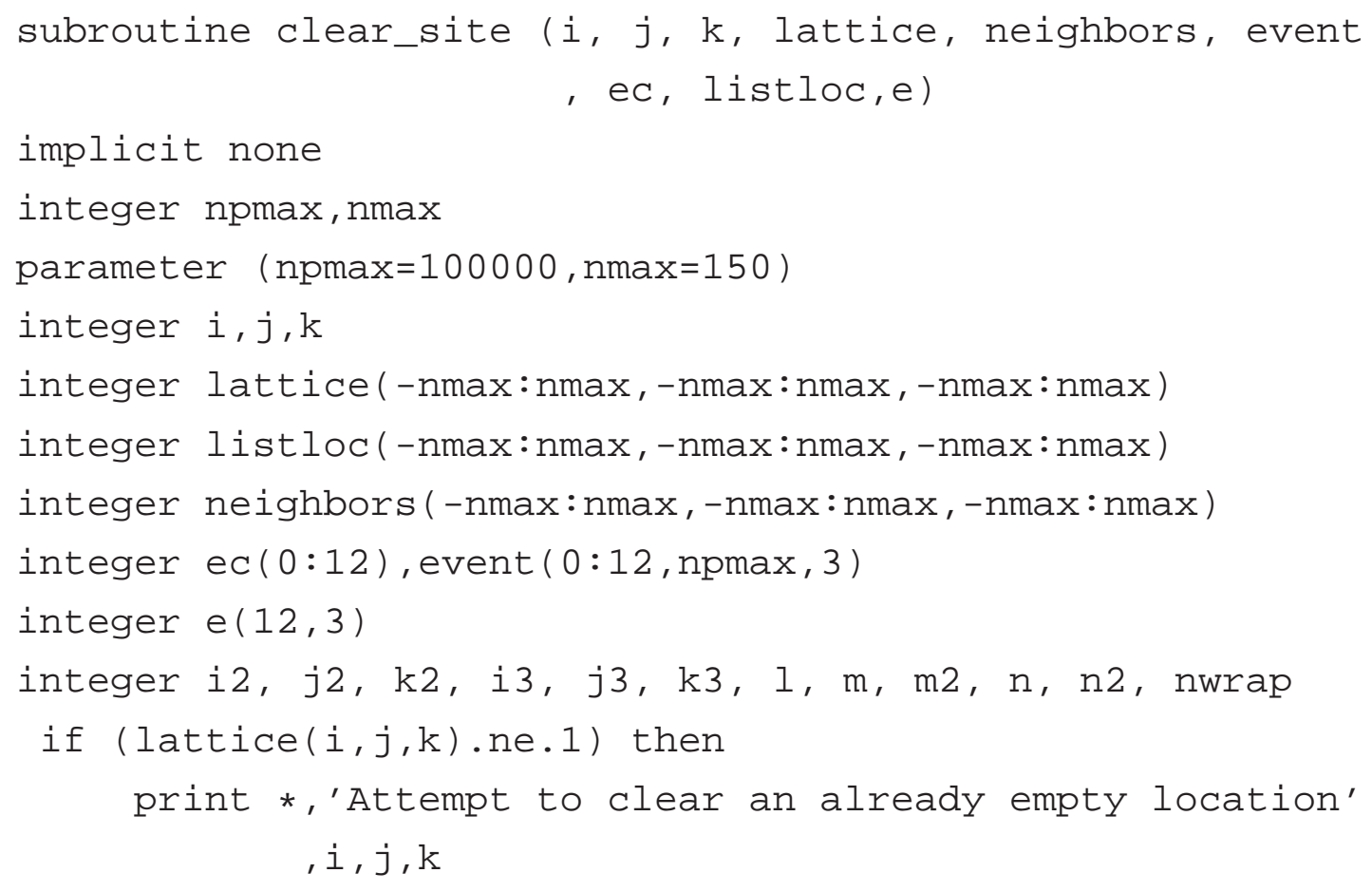




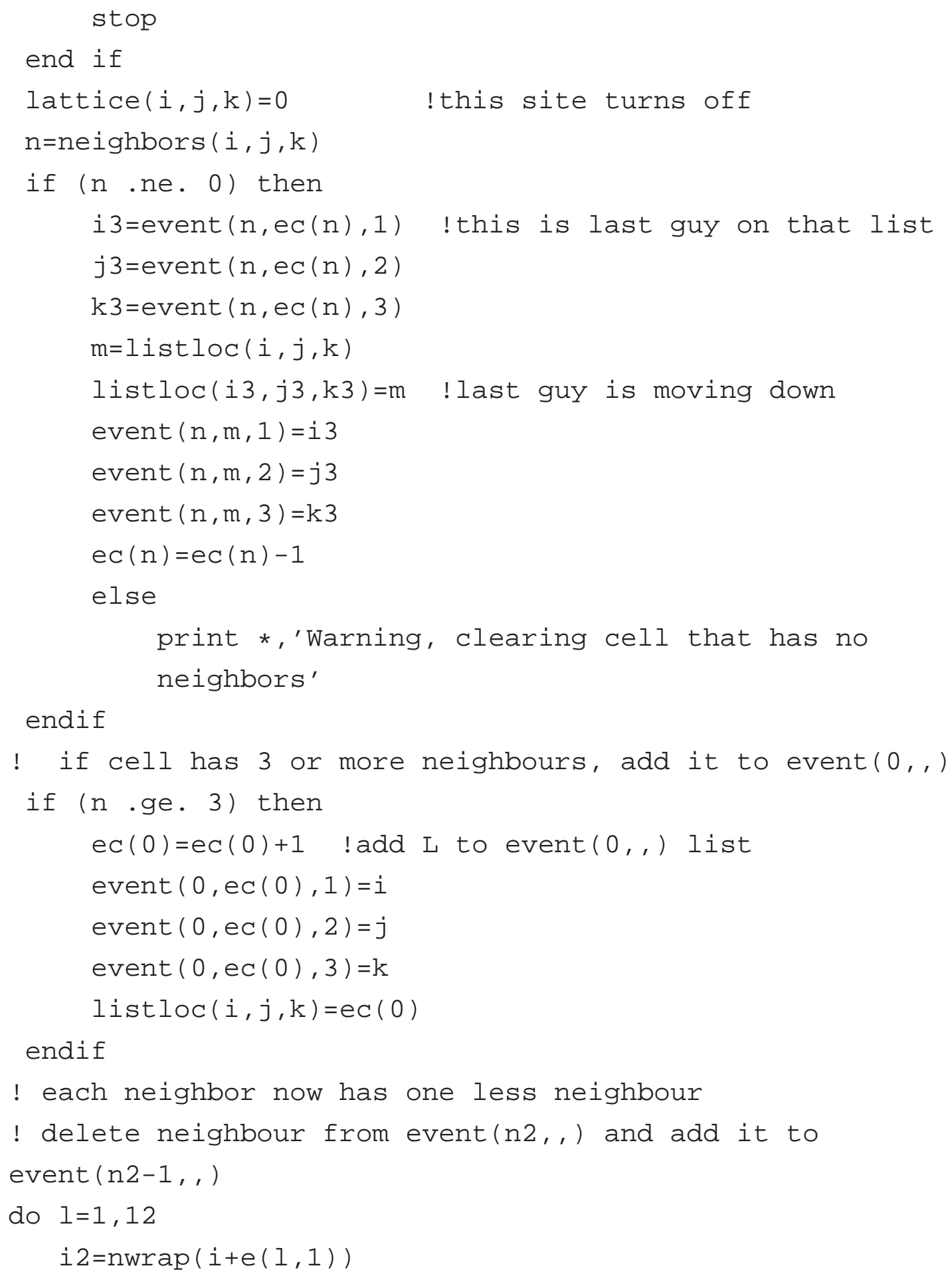




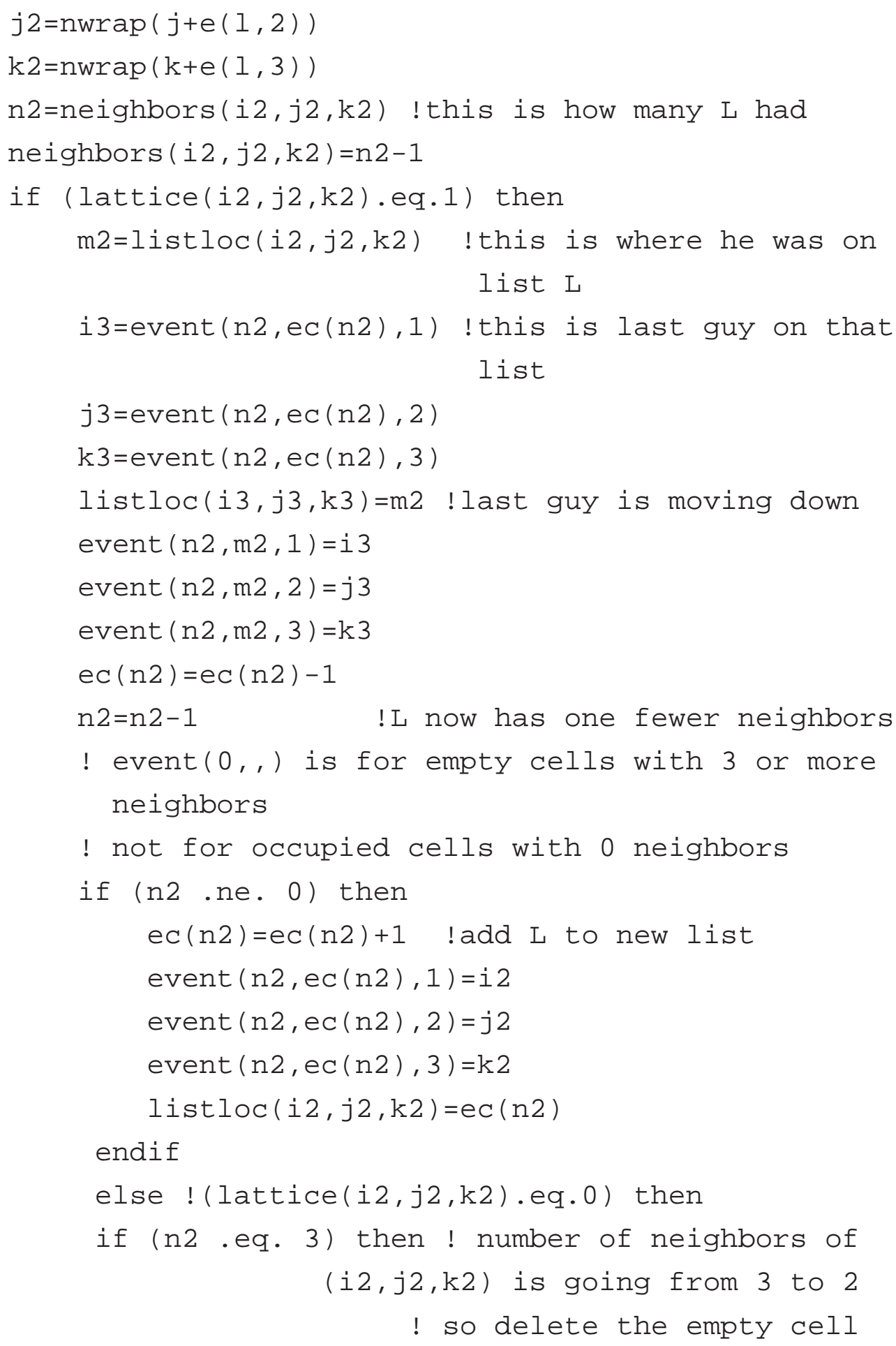




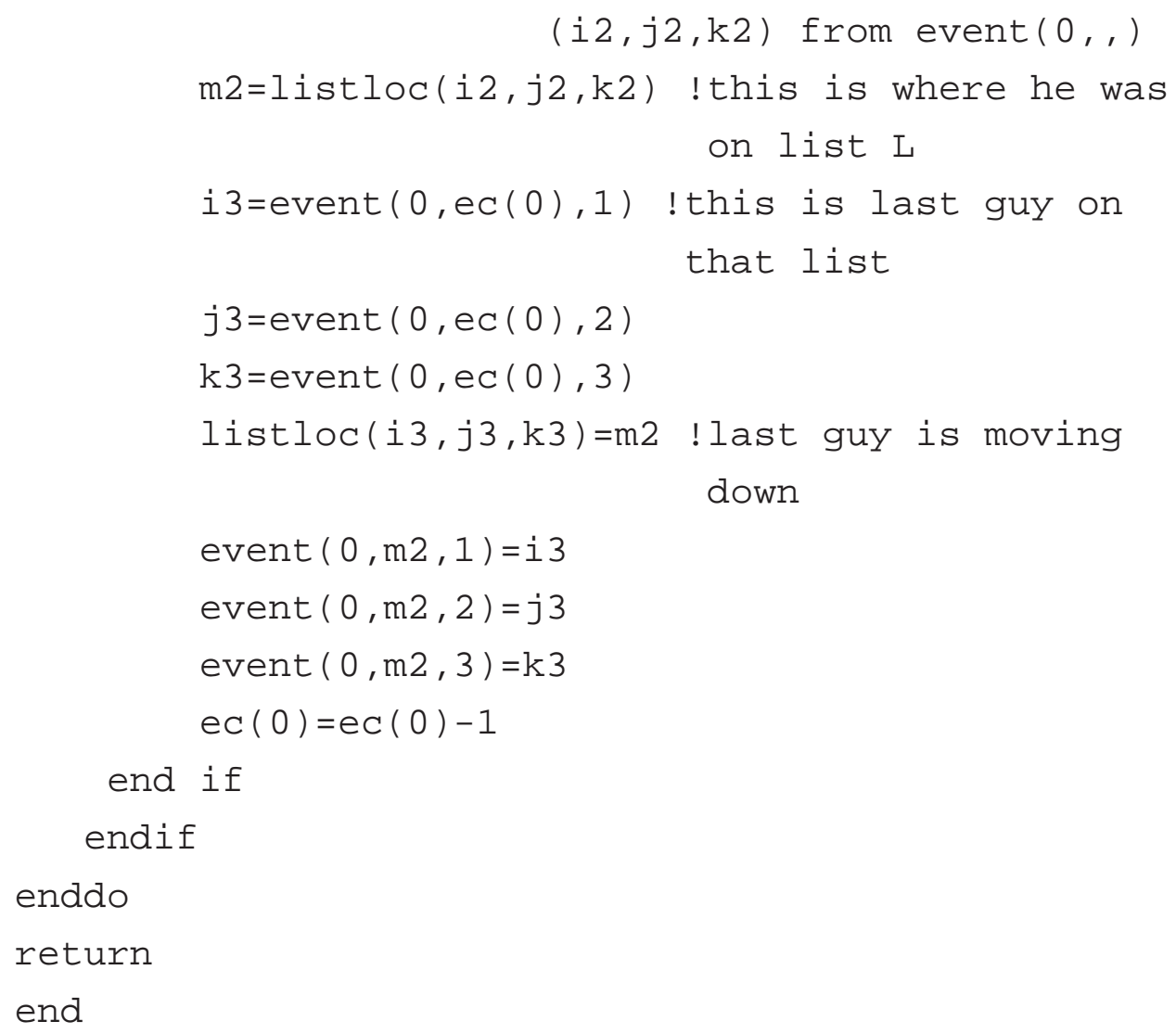

PART 2: Subroutine for setting an atom and updating the lattice site.

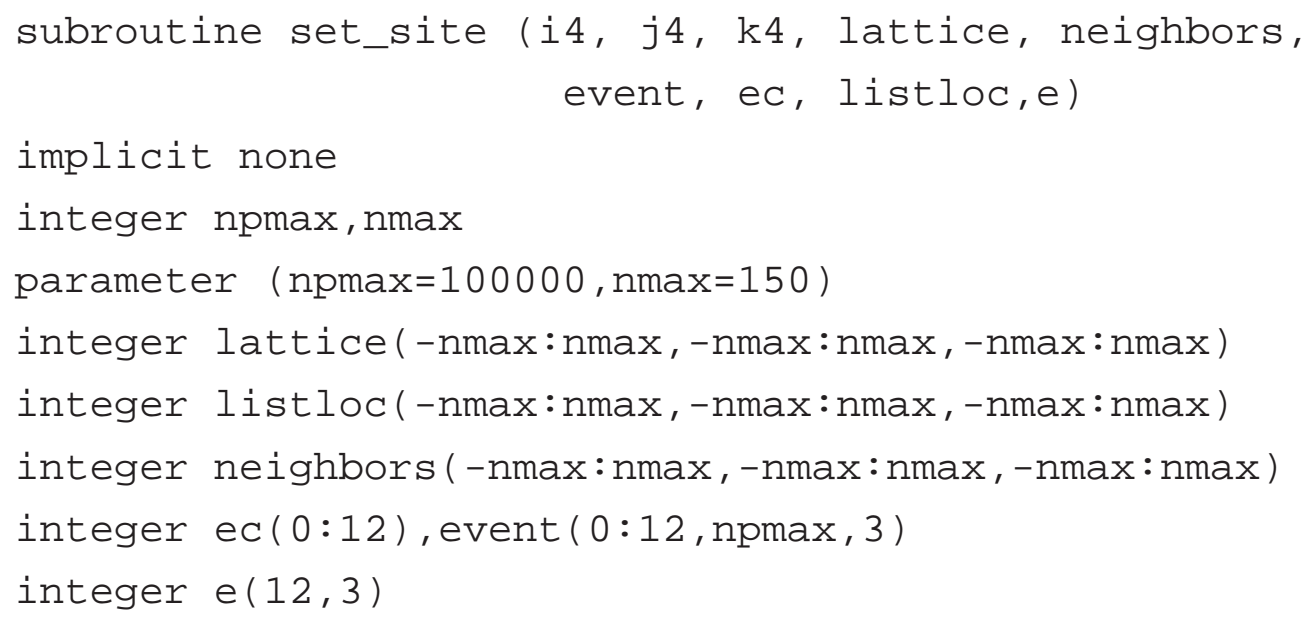




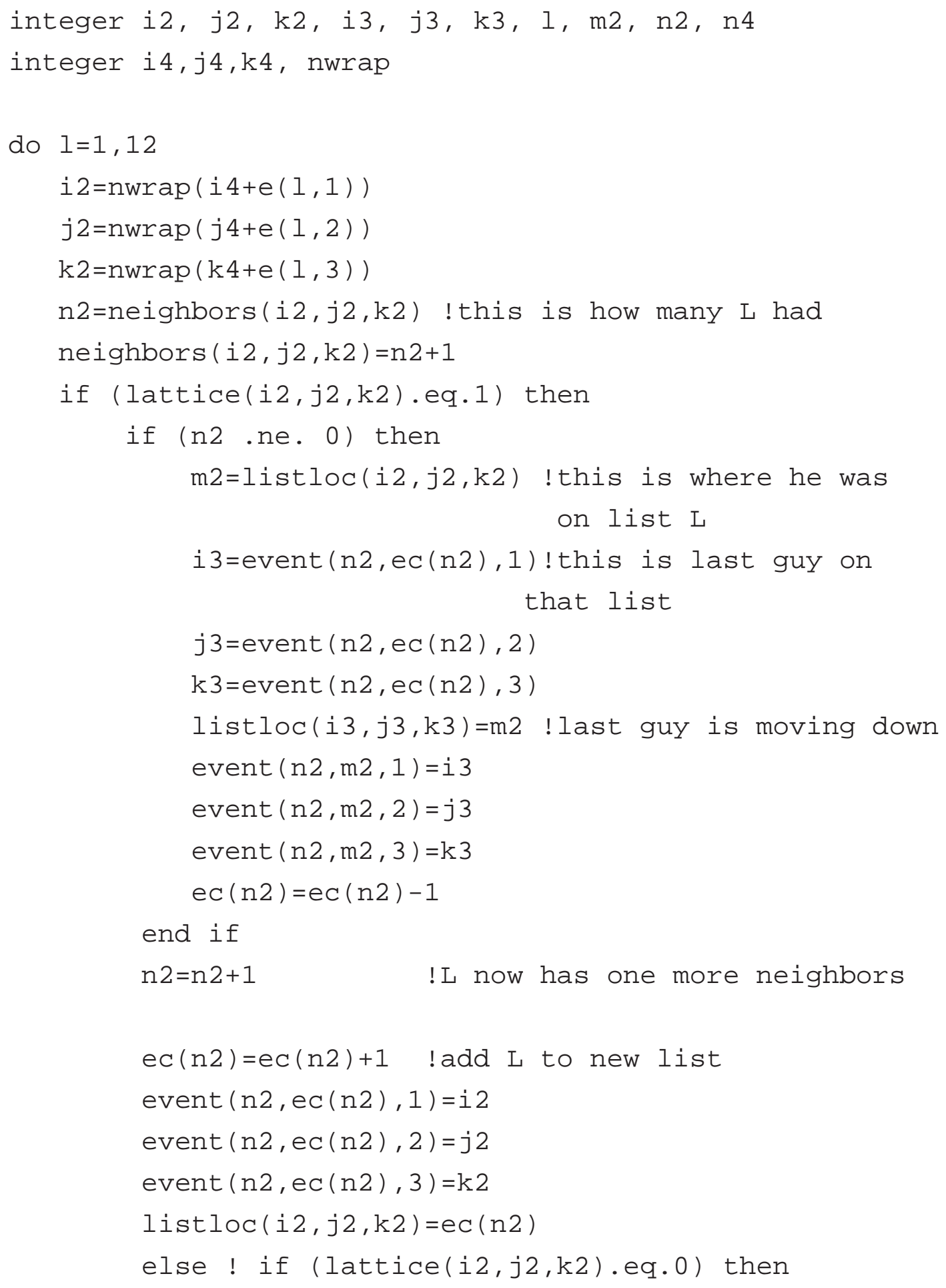


if (n2 .eq. 2) then ! empty cell (i2,j2,k2) is going from 2 to 3 neighbors ! so add it to event $(0$, , ) ec $(0)=e c(0)+1 \quad$ ! add $L$ to event $(0, r)$ list event $(0$, ec $(0), 1)=i 2$ event $(0, \operatorname{ec}(0), 2)=j 2$ event $(0$, ec $(0), 3)=\mathrm{k} 2$ listloc $(i 2, j 2, k 2)=e c(0)$

end if

endif

enddo

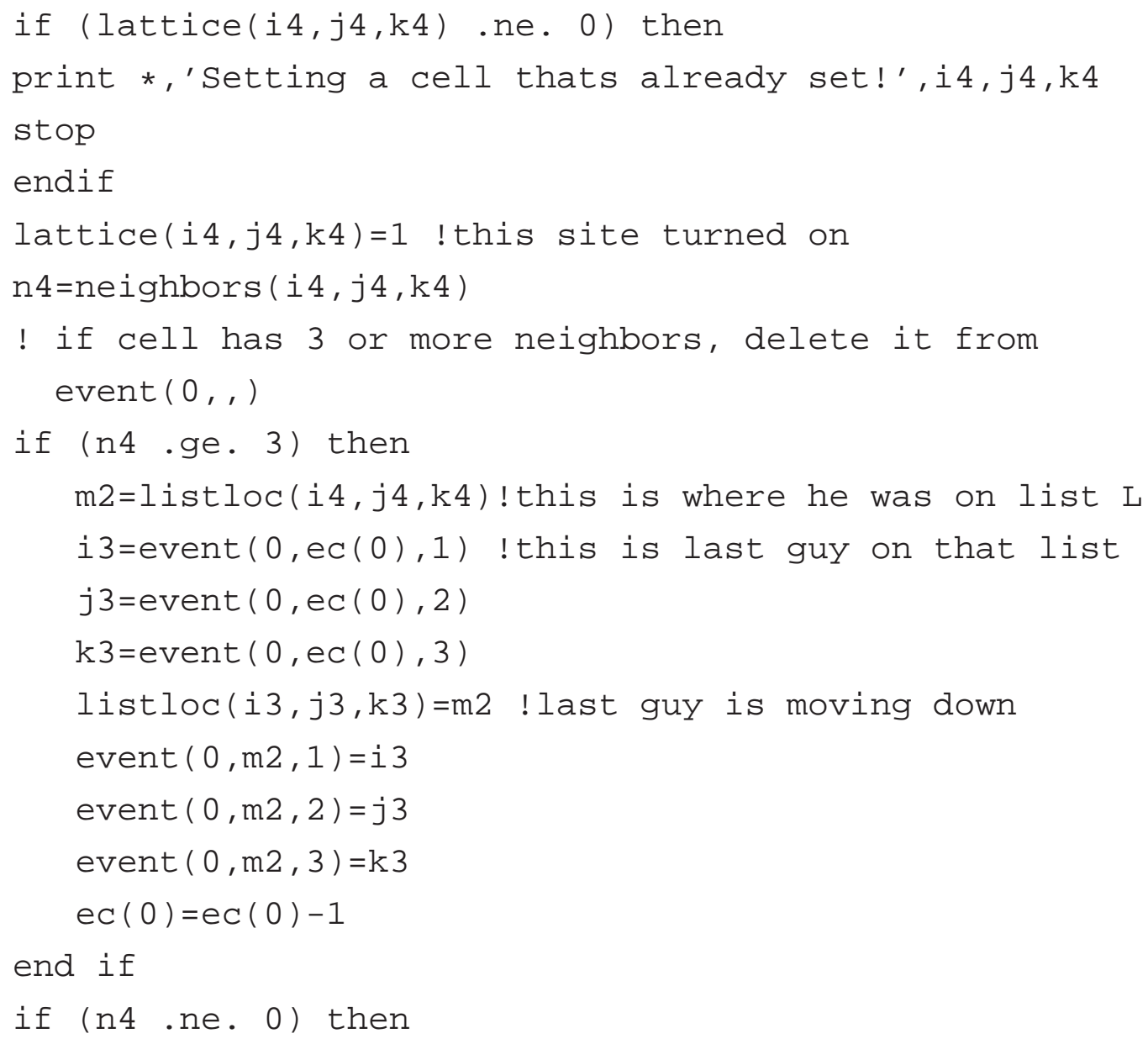




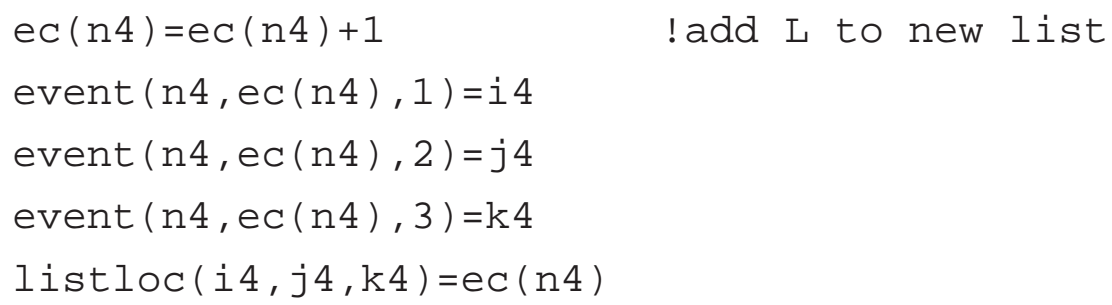

PART 3: Calculate the temperature interface $T_{M}$ and update the temperature and concentration fields.

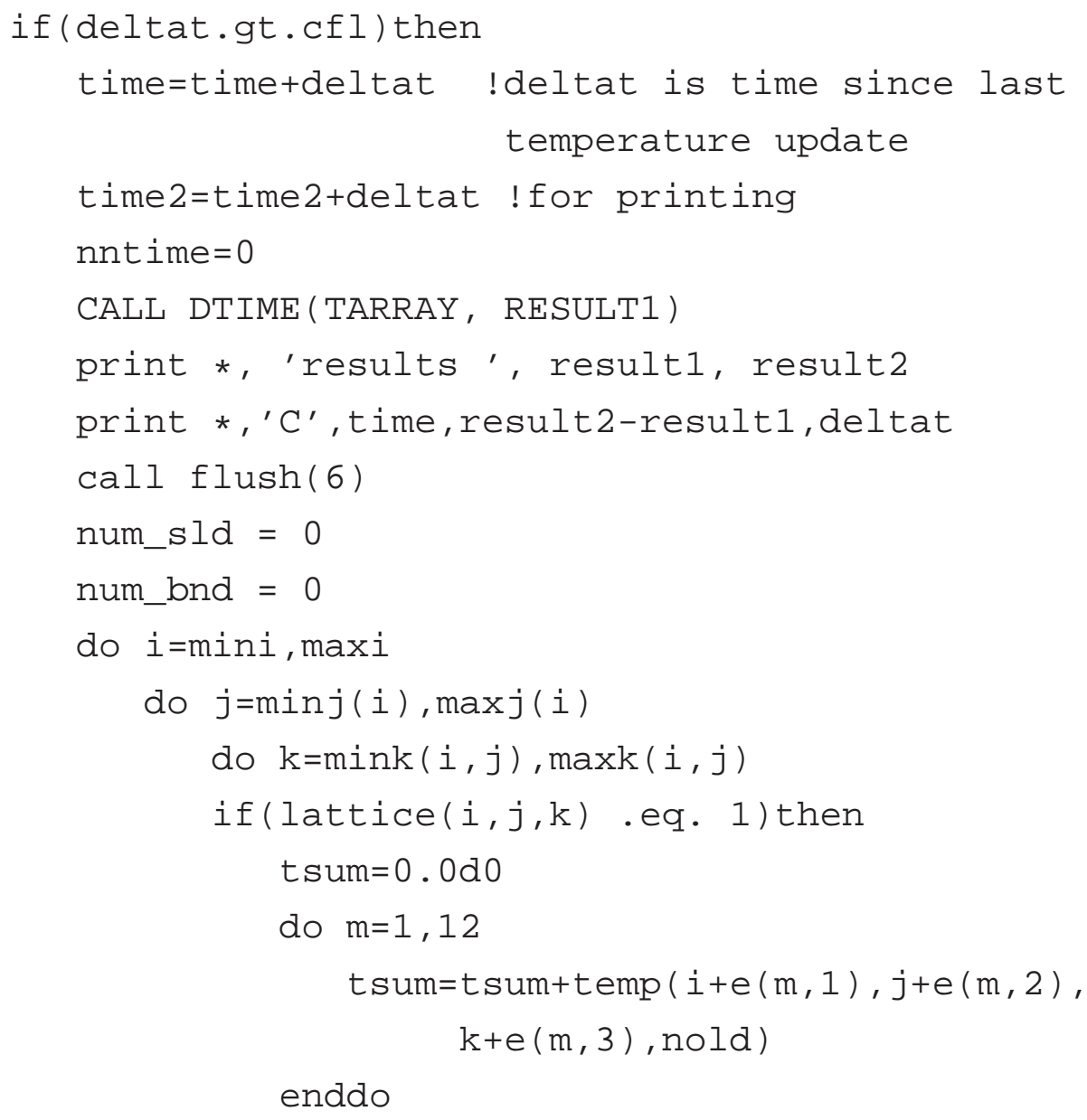




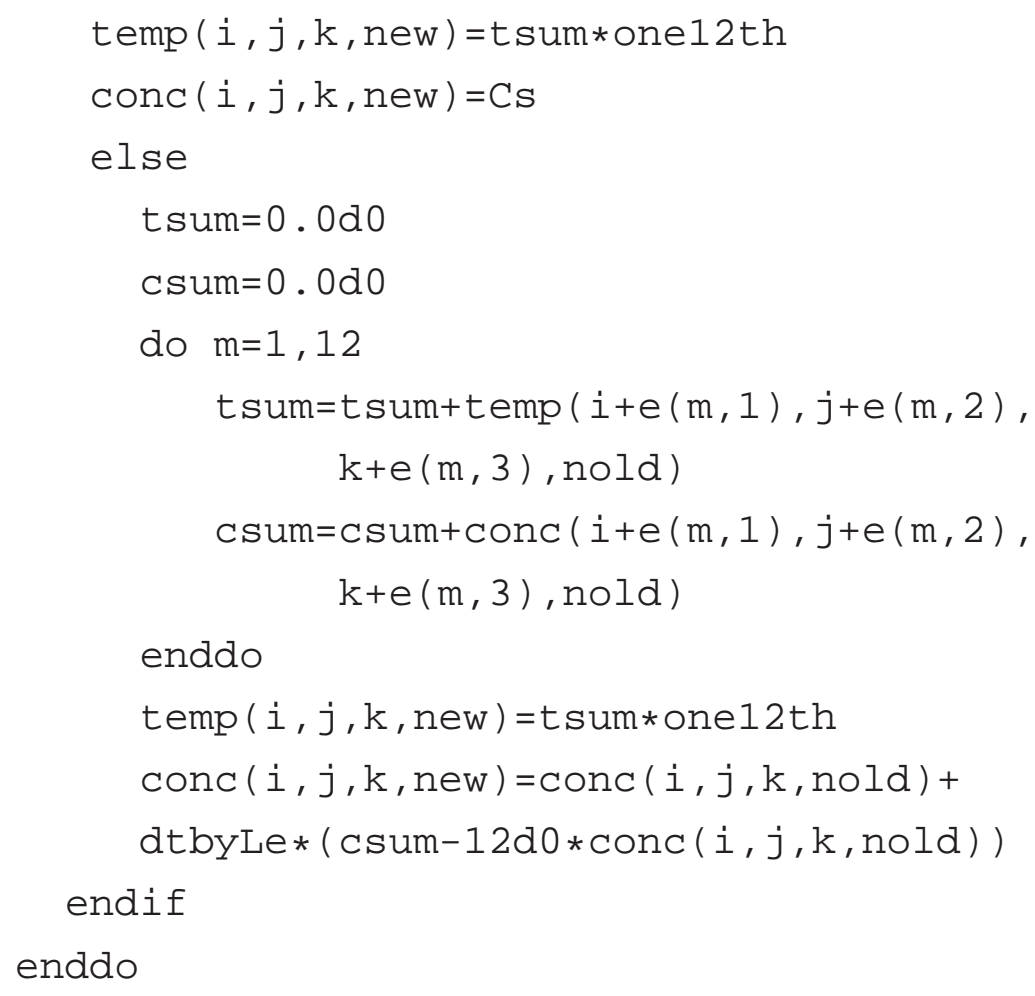




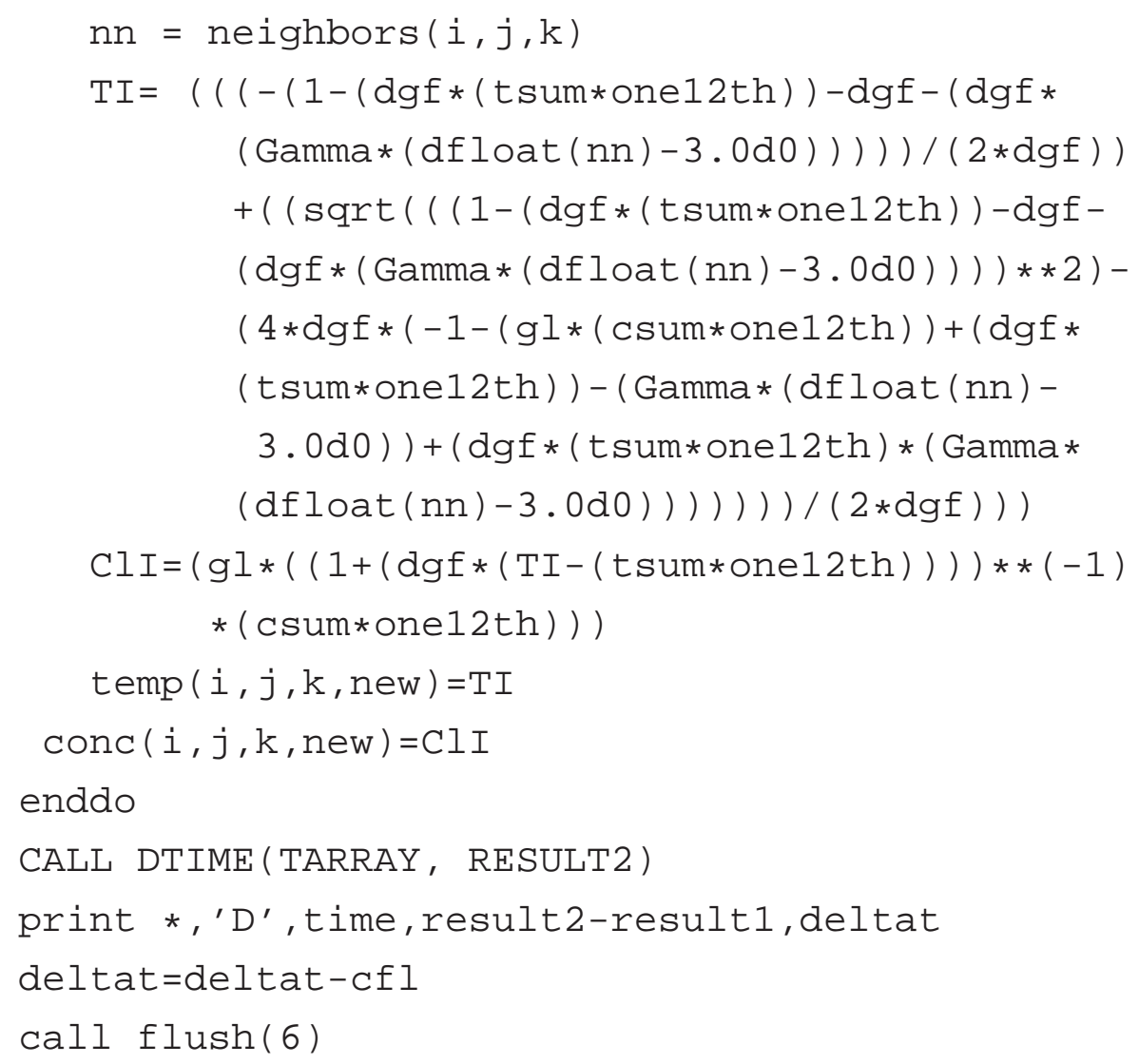

PART 4: Calculate the maximum height and tip velocity in $-z$ face

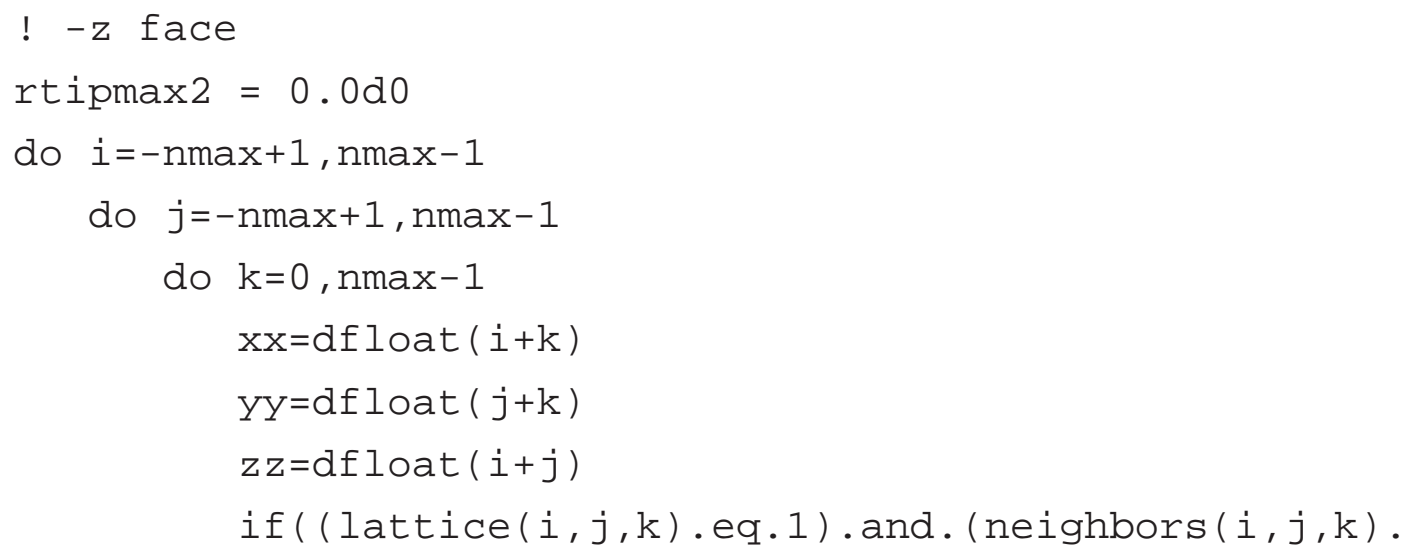




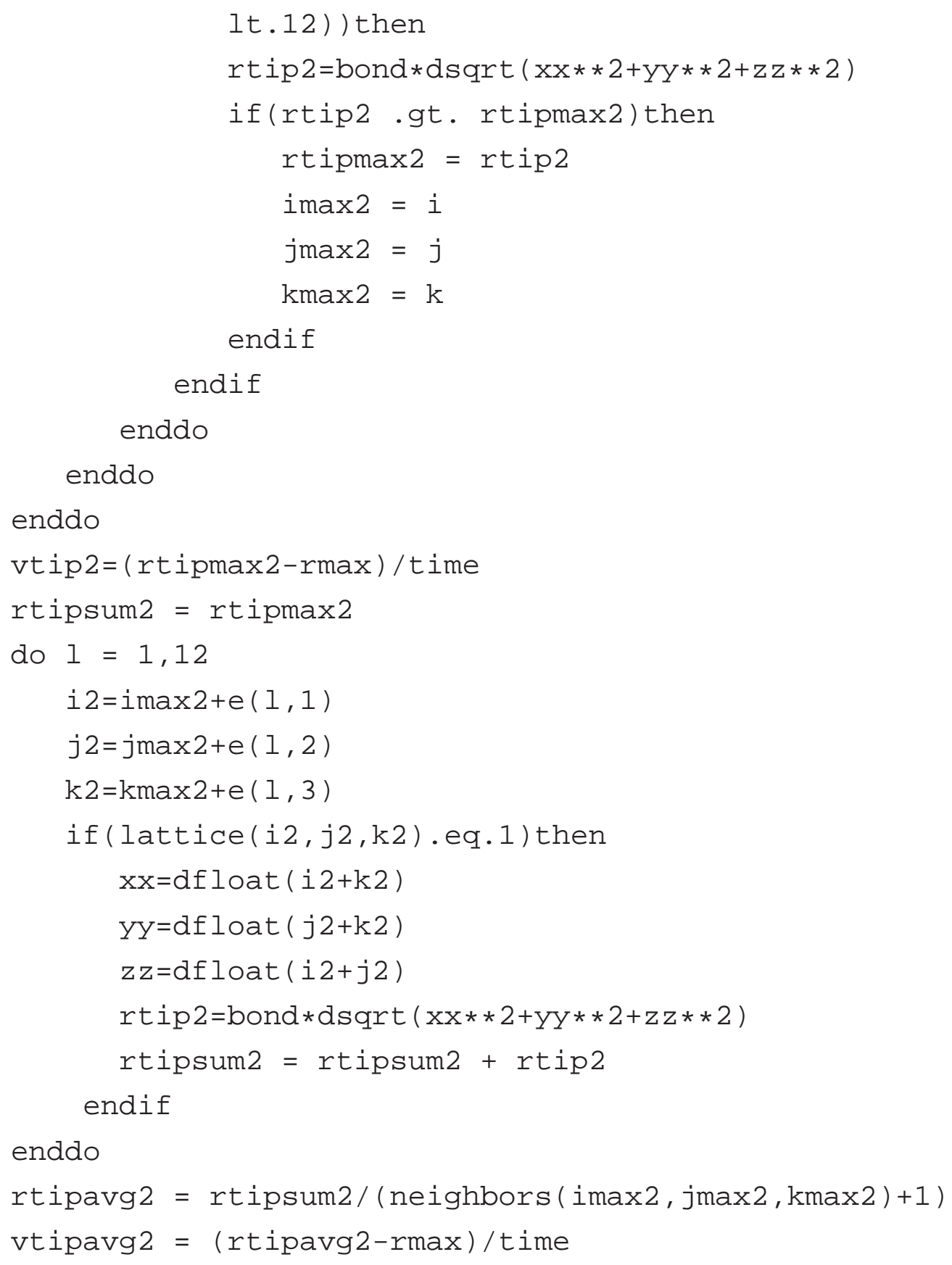




\section{Bibliography}

[1] C. B. Murray, C. R. Kagan, and M. G. Bawendi, "Synthesis and characterization of monodisperse nanocrystals and close-packed nanocrystal assemblies," Ann. Rev. Mater. Sci., vol. 30, no. 1, pp. 545610, 2000.

[2] W. J. Boettinger, J. A. Warren, C. Beckermann, and A. Karma, "Phase-field simulation of solidification 1," Ann. Rev. Mater. Research, vol. 32, no. 1, pp. 163-194, 2002.

[3] F. Gibou, R. Fedkiw, R. Caflisch, and S. Osher, "A level set approach for the numerical simulation of dendritic growth," J. Sci. Comput., vol. 19, no. 1, pp. 183-199, 2003.

[4] N. Provatas, M. Greenwood, B. Athreya, N. Goldenfeld, and J. Dantzig, "Multiscale modeling of solidification: Phase-field methods to adaptive mesh refinement," Int. J. Modern Phys. B, vol. 19, no. 31, pp. 4525-4566, 2005.

[5] M. Plapp and A. Karma, "Multiscale random-walk algorithm for simulating interfacial pattern formation," Phys. Rev. Lett., vol. 84, no. 8, pp. 1740-1743, 2000.

[6] M. Kotrla, "Numerical simulations in the theory of crystal growth," Comput. Phys. Communications, vol. 97, no. 1-2, pp. 82-100, 1996. 
[7] J. P. DeVita, L. M. Sander, and P. Smereka, "Multiscale kinetic monte carlo algorithm for simulating epitaxial growth," Phys. Rev. B, vol. 72, no. 20, pp. 205421-295428, 2005.

[8] G. Russo and P. Smereka, "Computation of strained epitaxial growth in three dimensions by kinetic monte carlo," J. Comp. Phys., vol. 214, no. 2, pp. 809-828, 2006.

[9] S. Zheng, W. Zhu, Y. F. Gao, G. M. Stocks, and Z. Zhang, "Kinetic monte carlo simulations of nanocolumn formation in twocomponent epitaxial growth," Appl. Phys. Lett., vol. 96, pp. 071913071920, 2010.

[10] S. Yip, Handbook of materials modeling. Kluwer Academic Pub, 2005.

[11] D. D. Vvedensky, "Multiscale modelling of nanostructures," J. Phys.: Condens. Matter, vol. 16, p. 1537, 2004.

[12] C. Li, Y. Y. Wu, H. Li, and X. F. Liu, "Morphological evolution and growth mechanism of primary mg2si phase in al-mg2si alloys," Acta Materialia, vol. 59.

[13] D. S. Coombes, C. R. A. Catlow, J. D. Gale, M. J. Hardy, and M. R. Saunders, "Theoretical and experimental investigations on the morphology of pharmaceutical crystals," J. Pharm. Sci., vol. 91, no. 7, pp. 1652-1658, 2002.

[14] J. H. Ter Horst, H. J. M. Kramer, G. M. Van Rosmalen, and P. J. Jansens, "Molecular modelling of the crystallization of polymorphs. part i: The morphology of hmx polymorphs," J. Cryst. Growth, vol. 237, pp. 2215-2220, 2002.

[15] I. M. Grimsey, J. C. Osborn, S. W. Doughty, P. York, and R. C. Rowe, "The application of molecular modelling to the interpretation of inverse gas chromatography data," J. Chromatogr. A., vol. 969, no. 1-2, pp. 49-57, 2002. 
[16] Y. Dirix, C. Bastiaansen, W. Caseri, and P. Smith, "Oriented pearlnecklace arrays of metallic nanoparticles in polymers: a new route toward polarization-dependent color filters," Advanced Materials, vol. 11, no. 3, pp. 223-227, 1999.

[17] R. Elghanian, J. J. Storhoff, R. C. Mucic, R. L. Letsinger, and C. A. Mirkin, "Selective colorimetric detection of polynucleotides based on the distance-dependent optical properties of gold nanoparticles," Science, vol. 277, no. 5329, pp. 1078-1081, 1997.

[18] Y. Y. Yu, S. S. Chang, C. L. Lee, and C. R. C. Wang, "Gold nanorods: electrochemical synthesis and optical properties," J. Phys. Chem. B, vol. 101, no. 34, pp. 6661-6664, 1997.

[19] C. Petit, A. Taleb, and M. P. Pileni, "Cobalt nanosized particles organized in a $2 \mathrm{~d}$ superlattice: synthesis, characterization, and magnetic properties," J. Phys. Chem. B, vol. 103, no. 11, pp. 1805-1810, 1999.

[20] V. F. Puntes, K. M. Krishnan, and A. P. Alivisatos, "Colloidal nanocrystal shape and size control: the case of cobalt," Science, vol. 291, no. 5511, pp. 2115-2117, 2001.

[21] M. C. Daniel and D. Astruc, "Gold nanoparticles: assembly, supramolecular chemistry, quantum-size-related properties, and applications toward biology, catalysis, and nanotechnology," Chem. Rev, vol. 104, no. 1, pp. 293-346, 2004.

[22] R. Narayanan and M. A. El-Sayed, "Changing catalytic activity during colloidal platinum nanocatalysis due to shape changes: electrontransfer reaction," J. Am. Chem. Soc, vol. 126, no. 23, pp. 7194-7195, 2004.

[23] S. Cheong, J. D. Watt, and R. D. Tilley, "Shape control of platinum and palladium nanoparticles for catalysis," Nanoscale, 2010. 
[24] A. C. Levi and M. Kotrla, "Theory and simulation of crystal growth," J. Phys.: Condens., vol. 9, pp. 299-344, 1997.

[25] A. P. J. Jansen, "An introduction to monte carlo simulations of surface reactions," Arxiv preprint cond-mat/0303028, 2003.

[26] A. F. Voter, "Introduction to the kinetic monte carlo method," Radiation Effects in Solids, vol. 235, pp. 1-23, 2007.

[27] I. Sunagawa, Crystals: growth, morphology, and perfection. Cambridge Univ Press, 2005.

[28] R. Hooke, Micrographia. Dover, New York, 1961.

[29] A. Holden and P. Morrison, Crystals and crystal growing. The MIT Press, 1982.

[30] W. K. Burton, N. Cabrera, and F. C. Frank, "The growth of crystals and the equilibrium structure of their surfaces," Philosophical Transactions of the Royal Society of London. Series A, Mathematical and Physical Sciences, vol. 243, no. 866, pp. 299-358, 1951.

[31] C. Kittel and P. McEuen, Introduction to solid state physics. Wiley New York, 1986.

[32] M. Hohage, M. Bott, M. Morgenstern, Z. Zhang, T. Michely, and G. Comsa, "Atomic processes in low temperature pt-dendrite growth on pt (111)," Phys. Rev. Lett., vol. 76, no. 13, pp. 2366-2369, 1996.

[33] M. Bott, M. Hohage, M. Morgenstern, T. Michely, and G. Comsa, "New approach for determination of diffusion parameters of adatoms," Phys. Rev. Lett., vol. 76, no. 8, pp. 1304-1307, 1996.

[34] Y. Doi, D. Matsunaka, and Y. Shibutani, "Thin film growth behaviors on strained fcc (111) surface by kinetic monte carlo," vol. 191, pp. 012017-012022, 2009. 
[35] K. Byrappa and T. Ohachi, Crystal growth technology. William Andrew, 2003.

[36] G. Liu, K. Chen, H. Zhou, J. Tian, C. Pereira, and J. M. F. Ferreira, "Fast shape evolution of tin microcrystals in combustion synthesis," Cryst. Growth, vol. 6, no. 10, pp. 2404-2411, 2006.

[37] A. Pimpinelli and J. Villain, Physics of crystal growth. Cambridge Univ Press, 1998.

[38] C. R. Henry, "Morphology of supported nanoparticles," Prog. Surf. Sci., vol. 80, no. 3-4, pp. 92-116, 2005.

[39] G. B. Arfken, H. J. Weber, and H. Weber, Mathematical methods for physicists. Academic press San Diego, 1995.

[40] J. J. Métois and J. C. Heyraud, "Sem studies of equilibrium forms: Roughening transition and surface melting of indium and lead crystals," Ultramicroscopy, vol. 31, no. 1, pp. 73-79, 1989.

[41] S. Swaminarayan, R. Najafabadi, and D. J. Srolovitz, "Polycrystalline surface properties from spherical crystallites: $\mathrm{Ag}$, au, cu and pt," Surf. Sci., vol. 306, no. 3, pp. 367-380, 1994.

[42] C. Mottet, J. Goniakowski, F. Baletto, R. Ferrando, and G. Treglia, "Modeling free and supported metallic nanoclusters: structure and dynamics," Phase Transitions, vol. 1, no. 2, pp. 101-113, 2004.

[43] L. D. Marks, "Particle size effects on wulff constructions," Surf. Sci., vol. 150, no. 2, pp. 358-366, 1985.

[44] W. E. Kingston, The physics of powder metallurgy. McGraw-Hil, New York, 1951.

[45] N. B. Ming and I. Sunagawa, "Twin lamellae as possible selfperpetuating step sources," J. Cryst. Growth, vol. 87, no. 1, pp. 13-17, 1988. 
[46] K. Kimoto and I. Nishida, "An electron microscope and electron diffraction study of fine smoke particles prepared by evaporation in argon gas at low pressures (ii)," Jap. J. Appl. Phys., vol. 6, p. 1047, 1967.

[47] J. G. Amar, "The monte carlo method in science and engineering," Comput. Sci. Eng., vol. 8, pp. 9-19, 2006.

[48] C. W. Gardiner, Handbook of stochastic methods: for physics, chemistry $\mathcal{E}$ the natural sciences. Springer, 2004.

[49] N. Metropolis, "The beginning of the monte carlo method," Los Alamos Science, vol. 15, pp. 125-130, 1987.

[50] J. Siepmann, An Introduction to the Monte Carlo Method for Particle Simulations, in Advances in Chemical Physics: Monte Carlo Methods in Chemical Physics, (eds I. Prigogine and S. A. Rice), vol. 105. John Wiley and Sons, Inc., 2007.

[51] F. F. Abraham and G. M. White, "Computer simulation of vapor deposition on two-dimensional lattices," J. Appl. Phys., vol. 41, pp. 1841-1849, 1970.

[52] G. H. Gilmer and P. Bennema, "Simulation of crystal growth with surface diffusion," J. Appl. Phys., vol. 43, pp. 1347-1360, 1972.

[53] G. H. Gilmer, "Growth on imperfect crystal faces:: I. monte-carlo growth rates," J. Cryst. Growth, vol. 36, no. 1, pp. 15-28, 1976.

[54] M. Bowker and D. A. King, "Adsorbate diffusion on single crystal surfaces:: I. the influence of lateral interactions," Surf. Sci., vol. 71, no. 3, pp. 583-598, 1978.

[55] D. A. Reed and G. Ehrlich, "Surface diffusivity and the time correlation of concentration fluctuations," Surf. Sci., vol. 105, no. 2-3, pp. 603-628, 1981. 
[56] P. A. Rikvold, "Simulations of a stochastic model for cluster growth on a square lattice," Phys. Rev. A, vol. 26, no. 1, pp. 647-650, 1982.

[57] E. S. Hood, B. H. Toby, and W. H. Weinberg, "Precursor-mediated molecular chemisorption and thermal desorption: The interrelationships among energetics, kinetics, and adsorbate lattice structure," Phys. Rev. Lett., vol. 55, no. 22, pp. 2437-2440, 1985.

[58] A. F. Voter, "Classically exact overlayer dynamics: Diffusion of rhodium clusters on rh (100)," Phys. Rev. B, vol. 34, no. 10, pp. 6819$6829,1986$.

[59] B. Meng and W. H. Weinberg, "Non-equilibrium effects on thermal desorption spectra," Surf. Sci., vol. 374, no. 1-3, pp. 443-453, 1997.

[60] K. A. Fichthorn and W. H. Weinberg, "Theoretical foundations of dynamical monte carlo simulations," J. Chem. Phys., vol. 95, no. 2, pp. 1090-1096, 1991.

[61] E. Kadossov and U. Burghaus, "Multi-site kinetic monte carlo simulations of thermal desorption spectroscopy data," Surf. Sci., vol. 603, no. 16, pp. 2494-2501, 2009.

[62] H. C. Kang and W. H. Weinberg, "Kinetic modeling of surface rate processes," Surf. Sci., vol. 299, pp. 755-768, 1994.

[63] B. Meng and W. H. Weinberg, "Monte carlo simulations of temperature programmed desorption spectra," J. Chem. Phys., vol. 100, pp. 5280-5290, 1994.

[64] C. Tahan, M. Suewattana, P. Larsen, S. Zhang, and H. Krakauer, "Kinetic monte carlo simulations of crystal growth in ferroelectric materials," AIP Conf. Proc., vol. 582, pp. 118-127, 2001.

[65] M. Itoh, "Atomic-scale homoepitaxial growth simulations of reconstructed iii-v surfaces," Surf. Sci., vol. 66, no. 3-5, pp. 53-153, 2001. 
[66] J. W. Evans, P. A. Thiel, and M. C. Bartelt, "Morphological evolution during epitaxial thin film growth: Formation of $2 \mathrm{~d}$ islands and $3 \mathrm{~d}$ mounds," Surf. Sci., vol. 61, no. 1-2, pp. 1-128, 2006.

[67] Z. Zhu, X. J. Zheng, and W. Li, "Multilayer growth of batio3 thin films via pulsed laser deposition: an energy-dependent kinetic monte carlo simulation," Appl. Surf. Sci., vol. 256, no. 20, pp. 58765881, 2010.

[68] F. Soisson, C. Becquart, N. Castin, C. Domain, L. Malerba, and E. Vincent, "Atomistic kinetic monte carlo studies of microchemical evolutions driven by diffusion processes under irradiation," $J$. Nuclear Materials, vol. 406, no. 1, pp. 55-67, 2010.

[69] S. D. Piersall and J. B. Anderson, "Direct monte carlo simulation of chemical reaction systems: Simple bimolecular reactions," J. Chem. Phys., vol. 95, no. 2, pp. 971-978, 1991.

[70] T. Vicsek and F. Family, "Dynamic scaling for aggregation of clusters," Phys. Rev. Lett., vol. 52, no. 19, pp. 1669-1672, 1984.

[71] C. C. Battaile and D. J. Srolovitz, "Kinetic monte carlo simulation of chemical vapor deposition," Ann. Rev. of Mater. Research, vol. 32, no. 1, pp. 297-319, 2002.

[72] R. Weinkamer, P. Fratzl, H. S. Gupta, O. Penrose, and J. L. Lebowitz, "Using kinetic monte carlo simulations to study phase separation in alloys," Phase Transitions, vol. 77, no. 5, pp. 433-456, 2004.

[73] S. M. Auerbach and H. I. Metiu, "Diffusion in zeolites via cage-tocage kinetics: Modeling benzene diffusion in na-y," J. Chem. Phys., vol. 105, pp. 3753-3759, 1996.

[74] M. J. Caturla, "Toward a predictive atomistic model of ion implantation and dopant diffusion in silicon," Comput. Mater. Sci., vol. 12, no. 4, pp. 319-332, 1998. 
[75] R. M. Ziff, E. Gulari, and Y. Barshad, "Kinetic phase transitions in an irreversible surface-reaction model," Phys. Rev. Lett., vol. 56, no. 24, pp. 2553-2556, 1986.

[76] K. Binder and D. W. Heermann, Monte Carlo simulation in statistical physics: an introduction. Springer, 2002.

[77] B. Lehner, M. Hohage, and P. Zeppenfeld, "Kinetic monte carlo simulation scheme for studying desorption processes," Surf. Sci., vol. 454, pp. 251-255, 2000.

[78] D. Frenkel and B. Smit, Understanding molecular simulation: from algorithms to applications. Academic Press, 2002.

[79] K. Reuter, First-Principles Kinetic Monte Carlo Simulations for Heterogeneous Catalysis: Concepts, Status and Frontiers. Wiley-VCH Weinheim, 2010.

[80] S. Nangia and B. J. Garrison, "Theoretical advances in the dissolution studies of mineral-water interfaces," Theor. Chem. Acc., vol. 127.

[81] A. Maiti and R. H. Gee, "Modeling growth, surface kinetics, and morphology evolution in petn," Propellants Explos. Pyrotech., vol. 34, no. 6, pp. 489-497, 2009.

[82] G. Henkelman and H. Jónsson, "A dimer method for finding saddle points on high dimensional potential surfaces using only first derivatives," J. Chem. Phys., vol. 111, pp. 7010-7023, 1999.

[83] J. Liu, C. Liu, and P. P. Conway, "Kinetic monte carlo simulation of kinetically limited copper electrocrystallization on an atomically even surface," Electrochimica Acta, vol. 54, no. 27, pp. 6941-6948, 2009.

[84] N. G. van Kampen, Stochastic processes in physics and chemistry. North Holland, 1992. 
[85] J. Hizanidis, The Master Equation. Available at http://wwwnlds.physik.tuberlin.de/ hizanidis /talks/mastermanu.pdf, 2002.

[86] A. Papoulis and S. U. Pillai, Probability, random variables, and stochastic processes. McGraw-Hill New York, 2002.

[87] H. C. Kang and W. H. Weinberg, "Dynamic monte carlo with a proper energy barrier: Surface diffusion and two-dimensional domain ordering," J. Chem. Phys., vol. 90, p. 2824, 1989.

[88] H. Kang and W. Weinberg, "Dynamic Monte Carlo simulations of surface-rate processes," Accounts of Chemical Research, vol. 25, no. 6, pp. 253-259, 1992.

[89] M. Suewattana, H. Krakauer, and S. Zhang, "Kinetic monte carlo simulations of crystal growth in ferroelectric alloys," Phys. Rev. B, vol. 71, no. 22, p. 224101, 2005.

[90] N. Metropolis, A. W. Rosenbluth, M. N. Rosenbluth, A. H. Teller, and E. Teller, "Equation of state calculations by fast computing machines," J. Chem. Phys., vol. 21, no. 6, pp. 1087-1092, 1953.

[91] K. Kawasaki, "Diffusion constants near the critical point for timedependent ising models. i," Phys. Rev., vol. 145, no. 1, pp. 224-230, 1966.

[92] K. Kawasaki, "Diffusion constants near the critical point for timedependent ising models. ii," Phys. Rev., vol. 148, no. 1, pp. 375-381, 1966.

[93] K. Kawasaki, "Diffusion constants near the critical point for timedependent ising models. iii. self-diffusion constant," Phys. Rev., vol. 150, no. 1, pp. 285-290, 1966. 
[94] W. Feller, An Introduction to Probability Theory and Its Applications. New York, Wiley, 1957.

[95] E. Cinlar, Introduction to stochastic processes. Prentice-Hall, Englewood Cliffs, 1975.

[96] A. B. Bortz, M. H. Kalos, and J. L. Lebowitz, "A new algorithm for monte carlo simulation of ising spin systems* 1," J. Comp. Phys., vol. 17, no. 1, pp. 10-18, 1975.

[97] J. L. Blue, I. Beichl, and F. Sullivan, "Faster monte carlo simulations," Phys. Rev. E, vol. 51, no. 2, pp. 867-868, 1995.

[98] P. A. Maksym, "Fast monte carlo simulation of mbe growth," Semiconductor Science and Technology, vol. 3, p. 594, 1988.

[99] T. P. Schulze, "Kinetic monte carlo simulations with minimal searching," Phys. Rev. E, vol. 65, no. 3, p. 36704, 2002.

[100] T. P. Schulze, "Efficient kinetic monte carlo simulation," J. Comp. Phys., vol. 227, no. 4, pp. 2455-2462, 2008.

[101] T. P. Schulze, "Simulation of dendritic growth into an undercooled melt using kinetic monte carlo techniques," Phys. Rev. E, vol. 78, no. 2, p. 20601, 2008.

[102] T. F. Edgar, S. W. Butler, W. J. Campbell, C. Pfeiffer, C. Bode, S. B. Hwang, K. S. Balakrishnan, and J. Hahn, "Automatic control in microelectronics manufacturing: Practices, challenges, and possibilities*1," Automatica, vol. 36, no. 11, pp. 1567-1603, 2000.

[103] V. Fiorentini, S. Oppo, and M. Scheffler, "Towards an understanding of surfactant action in the epitaxial growth of metals: The case of sb on ag (111)," Appl. Phys. A: Mater. Sci. Proc., vol. 60, no. 4, pp. 399402, 1995. 
[104] P. B. Barna and M. Adamik, "Fundamental structure forming phenomena of polycrystalline films and the structure zone models," Thin Solid Films, vol. 317, no. 1-2, pp. 27-33, 1998.

[105] P. Jensen, "Growth of nanostructures by cluster deposition: Experiments and simple models," Rev. Modern Phy., vol. 71, no. 5, pp. 16951735, 1999.

[106] L. H. Chan and E. I. Altman, "Nucleation and growth of Ag on Sbterminated Ge (1 0 0)," Surf. Sci., vol. 511, no. 1-3, pp. 229-239, 2002.

[107] T. Schmidt, M. Speckmann, J. Falta, T. O. Mentes, M. Niño, and A. Locatelli, "Ge growth on partially and entirely ag covered si (111)," J. Surf. Sci. Nanotechnology, vol. 8, no. 0, pp. 221-226, 2010.

[108] Z. Zhang and M. G. Lagally, Morphological organization in epitaxial growth and removal. World Scientific Pub Co Inc, 1998.

[109] J. Cho, S. G. Terry, R. LeSar, and C. G. Levi, "A kinetic monte carlo simulation of film growth by physical vapor deposition on rotating substrates," Mater. Sci. and Eng. A, vol. 391, no. 1-2, pp. 390-401, 2005.

[110] J. M. Pomeroy, J. Jacobsen, C. C. Hill, B. H. Cooper, and J. P. Sethna, "Kinetic monte carlo-molecular dynamics investigations of hyperthermal copper deposition on cu (111)," Phys. Rev. B, vol. 66, no. 23, p. 235412, 2002.

[111] L. Xu, C. T. Campbell, H. Jonsson, and G. Henkelman, "Kinetic monte carlo simulations of pd deposition and island growth on mgo (1 0 0)," Surf. Sci., vol. 601, no. 14, pp. 3133-3142, 2007.

[112] M. A. Gallivan, "Optimization, estimation, and control for kinetic monte carlo simulations of thin film deposition," 42nd IEEE Conference, vol. 4, pp. 3437-3442, 2004. 
[113] G. S. Kim, D. Klug, J. Tse, and P. Wu, "Kinetic monte carlo simulation of ta deposition on ta (100) surface," J. Mater. Sci., vol. 39, no. 4, pp. 1519-1522, 2004.

[114] S. Hendy, S. A. Brown, and M. Hyslop, "Coalescence of nanoscale metal clusters: Molecular-dynamics study," Phys. Rev. B, vol. 68, no. 24, pp. 241403-241407, 2003.

[115] D. Reinhard, B. D. Hall, D. Ugarte, and R. Monot, "Size-independent fcc-to-icosahedral structural transition in unsupported silver clusters: An electron diffraction study of clusters produced by inert-gas aggregation," Phys.l Rev. B, vol. 55, no. 12, pp. 7868-7881, 1997.

[116] N. Combe, P. Jensen, and A. Pimpinelli, "Changing shapes in the nanoworld," Phys. Rev. Lett., vol. 85, no. 1, pp. 110-113, 2000.

[117] P. Hanggi, P. Talkner, and M. Borkovec, "Reaction-rate theory: fifty years after kramers," Rev. Mod. Phys., vol. 62, no. 2, pp. 251-341, 1990.

[118] A. F. Voter and J. D. Doll, "Transition state theory description of surface self-diffusion: Comparison with classical trajectory results," $J$. Chem. Phys., vol. 80, pp. 5832-5839, 1984.

[119] P. Jensen and N. Combe, "Understanding the growth of nanocluster films," Comput. Mater. Sci., vol. 24, no. 1-2, pp. 78-87, 2002.

[120] A. La Magna, "Nanoisland shape relaxation mechanism," Surf. Sci., vol. 601, no. 2, pp. 308-314, 2007.

[121] M. Jose-Yacaman, C. Gutierrez-Wing, M. Miki, D. Q. Yang, K. N. Piyakis, and E. Sacher, "Surface diffusion and coalescence of mobile metal nanoparticles," J. Phys. Chem. B, vol. 109, no. 19, pp. 9703-9711, 2005. 
[122] T. H. Lim, D. McCarthy, S. C. Hendy, K. J. Stevens, S. A. Brown, and R. D. Tilley, "Real-time tem and kinetic monte carlo studies of the coalescence of decahedral gold nanoparticles," ACS Nano, vol. 3, no. 11, pp. 3809-3813, 2009.

[123] D. N. McCarthy and S. A. Brown, "Evolution of neck radius and relaxation of coalescing nanoparticles," Phys. Rev. B, vol. 80, no. 6, p. 64107, 2009.

[124] J. C. Ramirez, C. Beckermann, A. Karma, and H. J. Diepers, "Phasefield modeling of binary alloy solidification with coupled heat and solute diffusion," Phys. Rev. E, vol. 69, no. 5, p. 51607, 2004.

[125] Z. Wang, J. Wang, and G. Yang, "Phase-field investigation of effects of surface-tension anisotropy on deterministic sidebranching in solutal dendritic growth," Phys. Rev. E, vol. 78, no. 4, p. 42601, 2008.

[126] V. R. Voller, "An enthalpy method for modeling dendritic growth in a binary alloy," Int. J. Heat and Mass Transfer, vol. 51, no. 3-4, pp. 823834, 2008.

[127] M. Ohno and K. Matsuura, "Quantitative phase-field modeling for dilute alloy solidification involving diffusion in the solid," Phys. Rev. E, vol. 79, no. 3, p. 31603, 2009.

[128] W. W. Mullins and R. F. Sekerka, "Morphological stability of a particle growing by diffusion or heat flow," J.Appl. Phys., vol. 34, no. 2, pp. 323-329, 1963.

[129] J. M. Sullivan Jr, D. R. Lynch, and K. O’Neill, “Finite element simulation of planar instabilities during solidification of an undercooled melt," J. Comput. Phys., vol. 69, no. 1, pp. 81-111, 1987.

[130] H. S. Udaykumar, R. Mittal, and W. Shyy, "Computation of solidliquid phase fronts in the sharp interface limit on fixed grids," $J$. Comput. Phys., vol. 153, no. 2, pp. 535-574, 1999. 
[131] H. S. Udaykumar and L. Mao, "Sharp-interface simulation of dendritic solidification of solutions," Int. J. Heat and Mass Transfer, vol. 45, no. 24, pp. 4793-4808, 2002.

[132] H. S. Udaykumar, S. Marella, and S. Krishnan, "Sharp-interface simulation of dendritic growth with convection: benchmarks," Int. J. Heat Mass Transfer, vol. 46, no. 14, pp. 2615-2627, 2003.

[133] P. Zhao, M. Vénere, J. C. Heinrich, and D. R. Poirier, "Modeling dendritic growth of a binary alloy," J. Comp. Phys., vol. 188, no. 2, pp. 434-461, 2003.

[134] P. Zhao and J. C. Heinrich, "Front-tracking finite element method for dendritic solidification," J. Comput. Phys., vol. 173, no. 2, pp. 765796, 2001.

[135] D. Juric and G. Tryggvason, "A front-tracking method for dendritic solidification," J. Comput. Phys., vol. 123, no. 1, pp. 127-148, 1996.

[136] I. Steinbach, "Phase-field models in materials science," Modelling Simul. Mater. Sci. Eng., vol. 17, p. 073001, 2009.

[137] M. Plapp, "Phase-field simulations of crystal growth," 14 th International Summer School on Crystal Growth, vol. 1270, 2010.

[138] Y. Li, H. Geun Lee, and J. Kim, "A fast, robust, and accurate operator splitting method for phase-field simulations of crystal growth," $J$. Cryst. Growth.

[139] L. Tan and N. Zabaras, "A level set simulation of dendritic solidification with combined features of front-tracking and fixed-domain methods," J. Comput. Phys., vol. 211, no. 1, pp. 36-63, 2006.

[140] Y. Di and R. Li, "Computation of dendritic growth with level set model using a multi-mesh adaptive finite element method," J. Sci. Comput., vol. 39, no. 3, pp. 441-453, 2009. 
[141] S. Li, J. S. Lowengrub, and P. H. Leo, “Nonlinear morphological control of growing crystals," Physica D, vol. 208, no. 3-4, pp. 209-219, 2005.

[142] B. Echebarria, R. Folch, A. Karma, and M. Plapp, "Quantitative phase-field model of alloy solidification," Phys. Rev. E, vol. 70, no. 6, p. 61604, 2004.

[143] Y. Yang, J. W. Garvin, and H. S. Udaykumar, "Sharp interface numerical simulation of directional solidification of binary alloy in the presence of a ceramic particle," Int. J. Heat and Mass Transfer, vol. 51, no. 1-2, pp. 155-168, 2008.

[144] R. L. Parker, “Crystal growth mechanisms: Energetics, kinetics, and transport," Solid State Phys., vol. 25, pp. 151-299, 1970.

[145] M. Matsuoka and J. Garside, "Non-isothermal effectiveness factors and the role of heat transfer in crystal growth from solutions and melts," Chem. Eng. Sci., vol. 46, no. 1, pp. 183-192, 1991.

[146] M. Matsuoka and J. Garside, "The significance of mass and heat transfer during crystal growth from solutions and melts," Cryst. Growth, vol. 129, no. 3-4, pp. 385-393, 1993.

[147] J. Garside, "Industrial crystallization from solution," Chem. Eng. Sci., vol. 40, no. 1, pp. 3-26, 1985.

[148] P. Rudolph, "Transport phenomena of crystal growthheat and mass transfer," AIP Conference Proceedings, vol. 1270, p. 107, 2010.

[149] J. W. Mullin, Crystallisation. Butterworth \& Co Publishers Ltd, 1972.

[150] J. C. Brice and P. Rudolph, "Crystal Growth", in Ullmanns Encyclopedia of Industrial Chemistry. Wiley Online Library, 2007. 
[151] A. A. Chernov, Modern crystallography III.. Crystal growth. SpringerVerlag Berlin, 1984.

[152] D. T. J. Hurle, Handbook of crystal growth. North-Holland Amsterdam, 1993.

[153] M. Georgelin and A. Pocheau, "Characterization of cell tip curvature in directional solidification," Cryst. Growth, vol. 268, no. 1-2, pp. 272283, 2004.

[154] S. A. David, S. S. Babu, and J. M. Vitek, "Welding: Solidification and microstructure," J Miner. Met. Mater. Soc., vol. 55, no. 6, pp. 14-20, 2003.

[155] H. Honjo, S. Ohta, and Y. Sawada, "New experimental findings in two-dimensional dendritic crystal growth," Phys. Rev. Lett., vol. 55, no. 8, pp. 841-844, 1985.

[156] B. Nestler and A. Choudhury, "Phase-field modeling of multicomponent systems," Curr. Opin. Solid State Mater. Sci., vol. 15, pp. 93-105.

[157] H. M. Singer, I. Singer-Loginova, J. H. Bilgram, and G. Amberg, "Morphology diagram of thermal dendritic solidification by means of phase-field models in two and three dimensions," J. Cryst. Growth, vol. 296, no. 1, pp. 58-68, 2006.

[158] V. R. Voller, "A similarity solution for solidification of an undercooled binary alloy," Int. J. Heat and Mass Transfer, vol. 49, no. 11-12, pp. 1981-1985, 2006.

[159] J. C. Ramirez and C. Beckermann, "Examination of binary alloy free dendritic growth theories with a phase-field model," Acta Materialia, vol. 53, no. 6, pp. 1721-1736, 2005. 
[160] D. Sun, M. Zhu, S. Pan, and D. Raabe, "Lattice boltzmann modeling of dendritic growth in a forced melt convection," Acta Materialia, vol. 57, no. 6, pp. 1755-1767, 2009.

[161] M. F. Zhu and D. M. Stefanescu, "Virtual front tracking model for the quantitative modeling of dendritic growth in solidification of alloys," Acta Materialia, vol. 55, no. 5, pp. 1741-1756, 2007.

[162] A. Karma, "Phase-field formulation for quantitative modeling of alloy solidification," Phys. Rev. Lett., vol. 87, no. 11, p. 115701, 2001.

[163] R. C. Snyder and M. F. Doherty, "Faceted crystal shape evolution during dissolution or growth," AIChE J., vol. 53, no. 5, pp. 13371348, 2007.

[164] R. C. Snyder, S. Veesler, and M. F. Doherty, "The evolution of crystal shape during dissolution: Predictions and experiments," Cryst. Growth and Design, vol. 8, no. 4, pp. 1100-1101, 2008.

[165] F. C. Frank, "On the kinematic theory of crystal growth and dissolution processes," in Growth and perfection of crystals: proceedings, p. 411, Wiley, 1958.

[166] R. Lacmann, W. Franke, and R. Heimann, "The dissolution forms of single crystal spheres:: I. theory for the molecular-kinetics interpretation," J. Cryst. Growth, vol. 26, no. 1, pp. 107-116, 1974.

[167] M. Moore, "On the shapes of dissolved crystals," Mineralogical Magazine, vol. 50, no. 356, pp. 331-332, 1986.

[168] J. W. Gibbs, The scientific papers of J. Willard Gibbs. New York: Dover Publications Inc, 1961.

[169] A. C. Lasaga and A. E. Blum, "Surface chemistry, etch pits and mineral-water reactions," Geochimica et Cosmochimica Acta, vol. 50, no. 10, pp. 2363-2379, 1986. 
[170] A. C. Lasaga and A. Luttge, "A model for crystal dissolution," European Journal of Mineralogy, vol. 15, no. 4, p. 603, 2003.

[171] P. Meakin and K. M. Rosso, "Simple kinetic monte carlo models for dissolution pitting induced by crystal defects," J. Chem. Phys., vol. 129, p. 204106, 2008.

[172] Y. Zhang, J. P. Sizemore, and M. F. Doherty, "Shape evolution of 3dimensional faceted crystals," AIChE J., vol. 52, no. 5, pp. 1906-1915, 2006.

[173] P. Hartman and W. G. Perdok, "On the relations between structure and morphology of crystals: I," Acta Crystallographica, vol. 8, pp. 49$52,1955$.

[174] N. Cabrera and M. M. Levine, "On the dislocation theory of evaporation of crystals," Philosophical Magazine, vol. 1, no. 5, pp. 450-458, 1956.

[175] A. O'Hare, Numerical methods for physicists. University of Oxford, 2005.

[176] G. H. Gilmer, "Computer models of crystal growth," Science, vol. 208, no. 4442, p. 355, 1980.

[177] A. J. Gratz and P. Bird, "Quartz dissolution: Negative crystal experiments and a rate law," Geochimica et cosmochimica acta, vol. 57, no. 5, pp. 965-976, 1993.

[178] J. W. Tester, W. G. Worley, B. A. Robinson, C. O. Grigsby, and J. L. Feerer, "Correlating quartz dissolution kinetics in pure water from 25 to 625 c," Geochimica et cosmochimica acta, vol. 58, no. 11, pp. 24072420, 1994. 
[179] W. G. Worley, J. W. Tester, and C. O. Grigsby, "Quartz dissolution kinetics from 100-200 $\mathrm{c}$ as a function of ph and ionic strength," AIChE J., vol. 42, no. 12, pp. 3442-3457, 1996.

[180] M. Eßer, K. Morgenstern, G. Rosenfeld, and G. Comsa, “Dynamics of vacancy island coalescence on ag (111)," Surf. Sci., vol. 402, pp. 341345, 1998.

[181] F. A. Nichols and W. W. Mullins, "Surface (interface) and volumediffusion contributions to morphological changes driven by capillarity," Metall. Soc. AIME, vol. 233, pp. 1840-1847, 1965.

[182] M. J. Uttormark, M. O. Thompson, and P. Clancy, "Kinetics of crystal dissolution for a stillinger-weber model of silicon," Phys. Rev. B, vol. 47, no. 23, p. 15717, 1993.

[183] W. Bryc, "Applied probability and stochastic processes, lecture notes for 577/578 class," 1995, Available online.

[184] J. Doob, Stochastic processes. New York, 1953.

[185] M. Iosifescu, Finite Markov processes and their applications. Wiley New York, 1980.

[186] A. T. Bharucha-Reid, Elements of the Theory of Markov Processes and their Applications. Dover Pubns, 1997.

[187] H. Eyring, "The activated complex and the absolute rate of chemical reactions.," J. Chem. Phys., vol. 3, pp. 107-115, 1935.

[188] J. H. Beynon and J. R. Gilbert, Application of transition state theory to unimolecular reactions: an introduction. Wiley Chichester, 1984.

[189] K. J. Laidler and M. C. King, "Development of transition-state theory," J. Phys. Chem., vol. 87, no. 15, pp. 2657-2664, 1983. 
[190] D. G. Truhlar, B. C. Garrett, and S. J. Klippenstein, “Current status of transition-state theory," J. Phys. Chem., vol. 100, no. 31, pp. 1277112800, 1996. 